Finance and Economics Discussion Series Divisions of Research \& Statistics and Monetary Affairs Federal Reserve Board, Washington, D.C.

\title{
Estimates of Home Mortgage Originations, Repayments, and Debt On One-to-Four-Family Residences
}

\section{Alan Greenspan and James Kennedy}

2005-41

NOTE: Staff working papers in the Finance and Economics Discussion Series (FEDS) are preliminary materials circulated to stimulate discussion and critical comment. The analysis and conclusions set forth are those of the authors and do not indicate concurrence by other members of the research staff or the Board of Governors. References in publications to the Finance and Economics Discussion Series (other than acknowledgement) should be cleared with the author(s) to protect the tentative character of these papers. 


\title{
Estimates of Home Mortgage Originations, Repayments, and Debt On One-to-Four-Family Residences
}

\author{
Alan Greenspan and James Kennedy \\ Board of Governors of the Federal Reserve System
}

\section{September 2005}

Since 1997, when the Department of Housing and Urban Development discontinued its quarterly gross mortgage flow system, there has been no systematic attempt to disaggregate the net change in outstanding home mortgage debt into its constituent gross flows. Using a different approach, we have developed a system that reconciles the change in regular home mortgage debt with mortgage flows. The latter includes home purchase and refinance originations, and mortgage purchases, sales, and repayments for five types of mortgage originators and six categories of other mortgagees. In the process, we derive the sources of equity extraction from homes financed by mortgages.

Many people at the Federal Reserve assisted us in this work, including David Stockton, Robert Avery, Glenn Canner, Jennifer Attrep, Shannon Mok, Susan McIntosh, Wayne Passmore, Andreas Lehnert, Cathy Gessert, Kathleen Johnson, Ellen Merry, Charles Struckmeyer, William Ampeh, Allison Culpen, Andrew Paciorek, Greg Forte, and William Treacy. In addition, we received helpful comments from staff members at Fannie Mae, Freddie Mac, the Mortgage Bankers Association, the Bureau of the Census, the Federal Housing Administration, the Veterans Administration, the Department of Housing and Urban Development, the Federal Housing Finance Board, the Office of Federal Housing Enterprise Oversight, the Office of Thrift Supervision, Ginnie Mae, the National Association of Realtors, the National Association of Home Builders, National Mortgage News, and Inside Mortgage Finance. The views presented are solely those of the authors and do not necessarily represent those of the Federal Reserve Board or its staff. 


\section{Outline}

1. Introduction

2. Overview of the Mortgage Flows

3. Equity Extraction Financed by Home Mortgages

4. A Comparison of the Estimated Repayment Rates

5. Total Originations and Repayments

6. Estimating Originations in the Post-HMDA Quarters

7. Direction of Future Work

Appendixes
A. The Home Mortgage Disclosure Act Data
B. The Estimation of Repayments and Originations
C. Data Sources for Private MBS
D. Mortgage Companies
E. The Decomposition of Repayments
F. Mortgage Originations to Finance Home Purchases and to Refinance Existing Loans




\section{Introduction}

Since 1997, when the Department of Housing and Urban Development (HUD) discontinued its quarterly gross mortgage flow system, the Survey of Mortgage Lending Activity (SMLA), there has been no systematic attempt to disaggregate the net change in outstanding home mortgage debt into its constituent gross flows. ${ }^{1}$

Using a variety of data, we have developed a system that reconciles the change in regular home mortgage debt (that is, mortgages other than home equity and construction loans taken out to purchase a home or to refinance an existing loan) with mortgage flows for five types of mortgage originators and six categories of other mortgagees (enumerated below).

Four sources of data are central to our mortgage system: (1) regular mortgage debt outstanding (RMDO) from the Federal Reserve Board's flow of funds accounts (FOF); ${ }^{2}$ (2) mortgage flows reported under the Home Mortgage Disclosure Act (HMDA), which we estimate covers more than ninety percent of the volume of mortgage activity at banks and thrift institutions; (3) data from financial regulatory reports on RMDO at banks and thrift institutions that report under HMDA; and (4) data from the Government Sponsored Enterprises (GSEs), Ginnie Mae, and issuers of private mortgaged backed securities on mortgages they hold in portfolio, their securitized pools, and their purchases of mortgages. Estimation of the mortgage flows in our system is at the quarterly frequency; for clarity of presentation, we report most of our results at the annual frequency, but quarterly values are available upon request. Combining the Federal Reserve's quarterly changes of outstanding regular home mortgage debt with our estimate of total repayments

\footnotetext{
${ }^{1}$ Through the end of 1997, HUD's Survey of Mortgage Lending Activity (SMLA) provided survey-based estimates of mortgage originations, purchases, sales, repayments, and debt outstanding. Although still widely used as a benchmark for mortgage flows, especially originations, SMLA contained many problems. For example, it was not clear to what extent its data for banks and thrift institutions included direct subsidiaries. In addition, the survey methods were not the same across different types of originators (most notably, the method for banks and thrift institutions was much different from the method for mortgage companies). Furthermore, it was never clear to what extent SMLA included second liens. Such difficulties along with budgetary concerns led HUD to abandon its survey.

${ }^{2}$ Construction loans were estimated independently.
} 
(most of which is based on the implied repayments of mortgage pools) yields originations. We separate total originations into home-purchase and refinance loans based on the shares of those types of loans reported under HMDA.

Separately, we estimate the components of repayments: those resulting from refinancings, cancellation of debt by home sellers (separated into foreclosure sales and all other sales), unscheduled mortgage repayments, and scheduled amortization. Their derivation is presented in Appendix E.

The five types of originators in our system are commercial banks, thrift institutions regulated by the Office of Thrift Supervision (OTS), thrift institutions regulated by the Federal Deposit Insurance Corporation (which we refer to as savings banks), credit unions, and mortgage companies. Hereafter, we refer to the first four types collectively as depository institutions. The six categories of non-originators are the three GSEs (Fannie Mae, Freddie Mac, and the Federal Home Loan Banks), Ginnie Mae, issuers of private mortgage-backed securities, and all other types of mortgagees (including households, government agencies, pension funds, and life insurance companies). Our mortgage system includes estimates of cash-outs associated with refinancing, equity extracted through home sales, estimates of repayment rates across different types of mortgagees, and three measures of equity extraction.

\section{Overview of the Mortgage Flows}

This paper focuses on regular mortgages to purchase homes and to refinance existing loans in structures with one to four units and condominiums and co-ops in structures with five or more units.

To formalize our estimation procedure we first disaggregate the Flow of Funds estimate of the change in home mortgage debt into the following gross flows. 
$\begin{array}{ll}\text { Originations of } & \text { New homes (A) } \\ \text { regular mortgages: } & \text { Existing homes (B) } \\ & \text { Refinancings (C) } \\ & \text { Total originations (TO) }\end{array}$

Minus repayments Scheduled amortization (D)

of regular mortgages: Cancellation of refinanced loans $(\mathrm{E})$

Home sellers' mortgage cancellation (F)

Unscheduled repayments $(\mathrm{G})$

Total repayments (TR)

Equals: $\quad$ The change in regular mortgage debt outstanding (K)

Plus: $\quad$ The change in home equity loans outstanding (X)

Equals: The change in home mortgage debt outstanding excluding construction loans $(\mathrm{Q})$

Plus: $\quad$ The change in construction loans outstanding $(\mathrm{J})$

Equals: The change in home mortgage debt outstanding

The changes in home mortgage debt above, $\mathrm{K}$ and $\mathrm{X}$, are from Flow of Funds. In our mortgage system, total repayments (TR) is estimated from a number of sources, including mortgage pools for loans securitized by the GSEs, Ginnie Mae, and private conduits. We estimate repayments at banks and thrift institutions based on mortgage flows from HMDA and call report data on RMDO of HMDA reporters (see appendix B for a detailed description). As a check on our system, we calculate separate estimates of the components of total repayments, (D) through (G), which, conceptually, should sum to the total, but, as measured, results in a discrepancy, which is discussed below. Total 
originations is split into home purchase and refinance originations based on the shares of home purchase and refinance loans in HMDA (described further in appendix F).

\section{Equity Extraction Financed by Home Mortgages}

We define the extraction of equity on existing homes as the discretionary initiatives of home owners to convert equity in their homes into cash by borrowing in the home mortgage market. First, we hypothesize the change in home mortgage debt that would occur if homeowners took no discretionary actions to add or subtract from the debt on their home during a quarter. That change can be approximated in our system as mortgage originations to purchase new homes less scheduled amortization. The change in total home mortgage $\mathrm{debt}^{3}$ less that hypothetical quantity would, by construction, be equal to the discretionary extraction of home equity financed by home-mortgage debt in that quarter.

Accordingly, our definition of gross equity extraction (EE) may be expressed (in the notation above) as:

$$
E E=Q-[A-D]=(B-F)+(C-E)+(X-G)
$$

Figure 1 shows gross equity extraction and its three components. The term (B - F), mortgage originations on existing homes less home sellers' cancellation of outstanding debt, is the net change in mortgage debt resulting from the turnover of existing homes. Over the past decade and a half, (B - F) has been closely correlated with realized capital gains on the sale of homes. ${ }^{4}$ Cash outs through refinancing $(\mathrm{C}-\mathrm{E})$ and net extensions of

\footnotetext{
${ }^{3}$ Excluding construction loans.

${ }^{4}$ For homes sold in period $\mathrm{t}$, let $\mathrm{O}^{\mathrm{p}}\{\mathrm{t}\}$ denote the amount of the mortgages originally issued to finance the purchases of those homes and $\alpha$ the amount of those mortgages cumulatively paid down by the time of sale. Amortization and unscheduled repayments reduce the original mortgage balance; however, refinancings typically result in a higher loan balance. By definition, $\mathrm{O}^{\mathrm{p}}\{\mathrm{t}\}-\alpha=\mathrm{F}$. Let $\mathrm{w}_{\mathrm{s}}$ equal the amount of cash originally expended to purchase the homes that were sold in period $t$, including both down payments on mortgaged purchases and homes purchased with cash. $\mathrm{w}_{\mathrm{b}}$ denotes the comparable sum for buyers of homes in period t. Then, $\mathrm{O}^{\mathrm{p}}\{\mathrm{t}\}+\mathrm{w}_{\mathrm{s}}+\Pi=\mathrm{V}=$ value of homes sold in period $\mathrm{t}$, where $\Pi$ is the capital gain realized at the time of sale, defined as the sales price minus the purchase price. By definition, $\left(B+w_{b}\right)=V$. Thus,
} 
home equity loans less unscheduled repayments $(\mathrm{X}-\mathrm{G})$ are extractions of unrealized capital gains, but doubtless include some book-equity, as well. ${ }^{5}$

As discussed below, we also derive a "repayments discrepancy" equal to total repayments on RMDO estimated as an aggregate (described at the beginning of the next section and in appendix B) minus the sum of the estimated components (D to G).

As noted above, we define gross equity extraction as the change in RMDO minus new home originations plus scheduled amortization. Net equity extraction is defined as gross equity extraction less closing costs and other costs related to the extraction of home equity. ${ }^{6}$ These two measures are shown in figures 2 and 3, and in table 1 lines 21 to 24, both in levels and as a percent of disposable personal income.

\section{A Comparison of the Estimated Repayment Rates}

As mentioned above and described in detail in appendix B, we estimate separately repayment rates at banks, thrifts that report to OTS, savings banks, credit unions, the three GSEs, Ginnie Mae, and private MBS. We assume that the repayment rate at mortgage companies is equal to the average at depository institutions and credit unions

$$
\begin{aligned}
\mathrm{B}+\mathrm{w}_{\mathrm{b}} & =\Phi+\mathrm{w}_{\mathrm{s}}+\Pi \\
& =\mathrm{F}+\alpha+\mathrm{w}_{\mathrm{s}}+\Pi \\
\mathrm{w}_{\mathrm{b}}-\mathrm{w}_{\mathrm{s}}-\alpha & =\Pi+\mathrm{F}-\mathrm{B} \\
\left(\mathrm{w}_{\mathrm{b}}-\mathrm{w}_{\mathrm{s}}\right)-\alpha & =\Pi-(\mathrm{B}-\mathrm{F})
\end{aligned}
$$

That is, realized capital gains minus turnover extraction on existing homes equals:

(1) The excess of down payments and cash purchases by buyers over the down payments and cash purchases expended by current home sellers when originally acquiring their property, minus

(2) The extent of pay-down on the seller's original mortgage at the time the home was sold. In part because (1)-(2) is relatively small, the correlation between $\Pi$ and (B-F) from 1991:Q1 to 2004:Q4 was 0.92 .

${ }^{5}$ Based on industry sources, we have tentatively assumed that unscheduled repayments $(\mathrm{G})$ equal 0.001 of RMDO annually. Note that the quantity (X-G) is predominantly the change in home equity debt; $\mathrm{G}$, as we estimate it, averaged less than 2 percent of X from 1991 to 2004.

${ }^{6}$ The costs include commissions on homes sold (based on data in the National Income and Product Accounts), miscellaneous fees (which, in lieu of actual data, we assume to be fixed at 2 percent of originations of first liens and 1 percent of home equity loans), capital gains taxes paid on home sales, taxes paid when loans are refinanced (which we estimate as 1 percent of refinance originations), and points (based on data from the Federal Housing Finance Board's Monthly Interest Rate Survey). 
and that the repayment rate in the other mortgagees group is equal to the total average repayment rate.

As shown in figure 4 and in lines 76 to 88 of table 1, the total repayment rate averages 28 percent annually from 1991 to 2004, ranging from less than 14 percent in 1995 to more than 59 percent in 2003. As one would expect, the repayment rate shows a strong positive correlation with the share of refinancings in total originations, which, in turn, is correlated inversely with mortgage interest rates. For example, from 1991 to 2004 the correlation at the quarterly frequency between the repayment rate and the thirty-year fixed mortgage rate a quarter earlier is negative 0.71 .

The annual repayment rate on RMDO at the GSEs (line 84) averaged 26 percent during the 1991-2004 period, about 5 percentage points less than the average at banks and thrift institutions (line 78), apparently because depository institutions relative to the GSEs hold a much larger share of subprime loans and ARMs, which tend to repay faster than conforming loans. ${ }^{7}$ The repayment rate on private MBS averaged about 42 percent during the same period, more than 1-1/2 times larger than the average at the GSEs. ${ }^{8}$

\section{Total Originations and Repayments}

By construction, our estimate of total regular mortgage originations equals the sum of originations at the five types of originators. Equivalently, as noted above, total originations equals the change in total RMDO plus total repayments. Figure 5 shows

\footnotetext{
${ }^{7}$ One indication that subprime loans repay faster than other types of loans is their higher share of refinancing: According to the HMDA data, the refinance share for subprime loans averaged 67 percent from 1993 to 2004, compared with about 50 percent for both jumbo and conforming loans.

${ }^{8}$ Reasons why private MBS tend to repay faster than conforming loans at the GSEs are discussed in Bendt, and others, 2001; Bruskin and Sykes, 2001; Westoff and others, 2001; and Fan, 2001. Jumbo loans, which constitute close to one-half of non-agency MBS, probably repay at a faster rate than conforming loans because, owing to their larger size, borrowers have a greater monetary incentive to refinance them.

Borrowers with subprime loans, which constitute close to one-third of non-agency MBS, tend to refinance often in order to consolidate debts; in addition, subprime loans have a higher foreclosure rate than conforming loans. Finally, ARMs, which constitute a much larger share of private MBS than of the loans held or securitized by the GSEs, tend to repay faster than fixed-term loans.
} 
originations, repayments, and the change in RMDO and figure 6 shows the split of total originations into home purchase and refinance originations.

\section{Testing the Results}

Given the many sources of data uncertainty and the inevitable approximations we used in estimation, we sought to check the overall accuracy of our system, in part, by evaluating two of its key levers. First, we compared two, independently derived estimates of homepurchase originations as a share of the value of home purchases. Second, we compared total repayments to the sum of the separately estimated components (D) to (G). The first comparison exhibits an unresolved anomaly. The second adds confirmation to the result of a long series of calculations, each of which has an unknown error term.

\section{The Value of Homes Purchased Implied by Purchase Originations}

We estimate the mortgaged share of home purchases in units and the ratio of the average price of non-mortgaged compared with mortgaged properties from the 2000 Residential Finance Survey (RFS) and American Housing Surveys from 1989 to 2003. These imply that the mortgaged share in dollars trended up from close to 86 percent in 1991 to a bit more than 89 percent in recent years. ${ }^{9}$ We independently calculate the average loan-toprice ratio (LTP) of mortgaged homes as a weighted average of the LTPs for conventional loans and loans backed by the Federal Housing Administration (FHA) and the Veterans Administration (VA). ${ }^{10}$ The weighted LTP ratio averages about 80 percent during the 1991-2004 period, ranging from about 75-1/2 percent in 2003 to a bit more than 82 percent in 1994 (lines 18 to 20 of table 1 ).

Our estimates of home purchase originations adjusted by those ratios imply an overall value of home purchases. As shown in figure 7 and line 17 of table 1, this value is significantly lower, on average, than the value of purchases based on data from the

\footnotetext{
${ }^{9}$ The numerator of the mortgaged share excludes homes purchased with an assumed mortgage, which trended down over the sample period.

${ }^{10}$ The LTP for conventional loans is from the Federal Housing Finance Board's Monthly Interest Rate Survey. The LTPs for FHA and VA loans are based on data from those agencies and are at full coverage.
} 
Census Bureau and the National Association of Realtors (NAR). ${ }^{11}$ At this point, we cannot explain this large divergence, but hope in the future to narrow the discrepancy.

\section{The Mortgage Repayments Residual}

Total repayments in our mortgage system is equal to the sum of repayments across all of the categories of mortgagees. As noted above, we developed a separate estimate of total repayments equal to the sum of refinance repayments, sellers' debt cancellation, amortization, and unscheduled repayments (the latter three types hereafter are referred to as non-refinance repayments; see lines 92 to 105 of table 1 and figure 8). The "repayments discrepancy," defined as total repayments in our mortgage system minus the sum of the four components provides an indication of the overall accuracy of total repayments. The residual averaged close to zero over the 1991 to 2004 period (lines 99 and 105). Nevertheless, the annual discrepancies deviate considerably from zero in some years, ranging from 1.9 percent of beginning-of-year RMDO in 1991 to -1.4 percent in 2002.

\section{Estimating Originations in the Post-HMDA Quarters}

For the quarters beyond the range of the HMDA, originations and repayments are available only at the aggregate level. We employ data on the change in RMDO and repayments on mortgages held or securitized by the GSEs, Ginnie Mae, and private MBS conduits. We estimate repayments at depository institutions and credit unions by multiplying the level of their RMDO at the end of the previous quarter by a projected

\footnotetext{
${ }^{11}$ Let $\mathrm{h}_{\mathrm{m}}$ : number of homes purchased that are mortgaged $(\mathrm{m}), \mathrm{h}_{\mathrm{n}}$ : number of homes purchased without a mortgage (n), $p_{n}$ : price of mortgaged homes, $p_{n}$ : price of non-mortgaged homes, $\mathrm{O}^{\mathrm{p}}$ : home purchase originations, and $\mathrm{V}$ the total value of homes purchased. Then, $\mathrm{V} \equiv\left(\mathrm{h}_{\mathrm{m}}{ }^{*} \mathrm{p}_{\mathrm{m}}\right)+\left(\mathrm{h}_{\mathrm{n}}{ }^{*} \mathrm{p}_{\mathrm{n}}\right)$

Define $\alpha \equiv\left(\mathrm{p}_{\mathrm{n}} / \mathrm{p}_{\mathrm{m}}\right), \beta \equiv\left(\mathrm{h}_{\mathrm{n}} / \mathrm{h}_{\mathrm{m}}\right)$, LTV $\equiv\left(\mathrm{O}^{\mathrm{p}} / \mathrm{V}\right)$. Substituting, $\mathrm{V}=\left(\mathrm{h}_{\mathrm{m}}{ }^{*} \mathrm{p}_{\mathrm{m}}\right)+\left(\beta \mathrm{h}_{\mathrm{m}} * \alpha \mathrm{p}_{\mathrm{m}}\right)=\left(\mathrm{h}_{\mathrm{m}}{ }^{*} \mathrm{p}_{\mathrm{m}}\right) *(1+\beta \alpha)=\left(\mathrm{O}^{\mathrm{p}} / \mathrm{LTV}\right) *(1+\beta \alpha)$

Therefore, $\{(1+\beta \alpha) / \mathrm{LTV}\}=\left(\mathrm{V} / \mathrm{O}^{\mathrm{p}}\right)$. For example, in 2004:Q2, $\beta=0.1765, \alpha=0.7800, \mathrm{O}^{\mathrm{p}}=\$ 353.7, \mathrm{LTV}=0.7535$. Thus, the implied value of total purchases consistent with our originations and the other parameters in the equations above is $\$ 534.0$ billion. The comparable estimate of the value of homes purchased based on Census and NAR data is $\$ 571.5$ billion.
} 
repayment rate, estimated from an equation with the following explanatory variables: the repayment rate at the GSEs and Ginnie Mae and the levels of the thirty-year fixed mortgage rate and the one-year adjustable mortgage rate. ${ }^{12}$ Table 2 shows estimates of originations in the first quarter of this year using the method described above.

\section{Direction of Future Work}

Because of multiple sources of data, we inevitably uncover contradictory results that require judgments of the relative accuracy of different approaches. These contradictions will be the main focus of additional efforts at fine-tuning and expanding the system. We intend to address the following gaps and anomalies in the data and shortfalls in our estimation procedures:

- Our adjustments to HMDA data on sales of mortgages (described in appendix A) assume that the quarterly adjustment factors that correct for both truncation and timing-of-sale bias are the same in each year, but those factors vary over time. For example, originators are less likely to sell ARMs than fixed-rate loans; in itself, this suggests that the factors used to correct for truncation bias should vary inversely with the ARM share. In addition, the share of loans both acquired and sold in the current quarter may vary directly with the volume of acquisitions. For example, during the refinancing boom of 2002 and 2003, the lag between the time of origination and sale may have stretched out because of bottlenecks in the industry.

- HMDA sales exclude loans originated in one year and sold in a subsequent year ("seasoned loans"). Our adjustments to HMDA sales currently do not contain a correction for sales of seasoned loans, although we hope to incorporate such an adjustment in the future. ${ }^{13}$ In itself, the exclusion of sales of seasoned loans imparts an upward bias to repayments, and thus to originations.

\footnotetext{
${ }^{12}$ A description of and summary statistics for the model described in this section are available upon request. We use a separate system of equations to project the refinance share.

${ }^{13}$ OFHEO is developing a new database that may provide data on seasoned-loan sales. Specifically, OFHEO has asked Fannie and Freddie to provide detailed information on the loans they have purchased since 1979, including the date the seller acquired the loan.
} 
- FOF bases its estimate of RMDO at mortgage companies on SMLA through 1997; since the demise of SMLA, FOF has held this sector's RMDO constant. However, the mortgage flows in the HMDA data suggest that RMDO at mortgage companies has trended up since 1997 (see appendix D). More generally, if the typical lag between the time of origination and sale is approximately stable, a rise in originations implies an increase in the number of loans on the books of mortgage companies awaiting sale, and thus a higher level of RMDO. Federal Reserve Board staff hope to develop data sources such as Securities and Exchange Commission filings and annual reports filed by large mortgage companies that will improve measurement of RMDO at mortgage companies.

- HMDA forms ask respondents to report all loans sold during the year, but in a few documented cases in the early 1990s, respondents mistakenly understated sales (those respondents subsequently submitted corrected reports). ${ }^{14}$ Underreporting of sales apparently has not been significant in recent years.

- Our mortgage system measures flows of regular mortgages. However, in addition to regular mortgages used to purchase homes or to refinance existing loans, HMDA includes second liens taken out at the time of purchase; these second liens sometimes are referred to as "piggyback loans." Beginning in 2004, HMDA identifies piggybacks loans. Before then, we filtered out piggybacks by excluding all home purchase loans for less than $\$ 25,000$. However, the average size of piggyback loans for home purchase in 2004 was $\$ 46,000$, suggesting that the threshold should be raised above $\$ 25,000$ in recent years. ${ }^{15}$

- We do not separately estimate regular mortgage originations on debt-free properties. According to the RFS, such loans represent a small share of RMDO - As mentioned in appendix B, we assumed that none of the members of the other mortgagees group originates loans. In the future, we hope to find data that will allow us to estimate the originations in the category.

\footnotetext{
${ }^{14}$ For example, see Scheessele (1998). The paper contains references to other studies that discuss the potential underreporting of HMDA sales.

${ }^{15}$ Piggyback loans often are used to achieve an 80 percent loan-to-price ratio to avoid paying for mortgage insurance or to reduce the size of the loan below the conforming threshold. Appendix B describes how the inclusion of piggyback loans would affect originations in our mortgage system.
} 
Figure 1

The Components of Gross Equity Extraction

Billions of dollars

(1991:Q1-2005:Q1, seasonally adjusted annual rate)

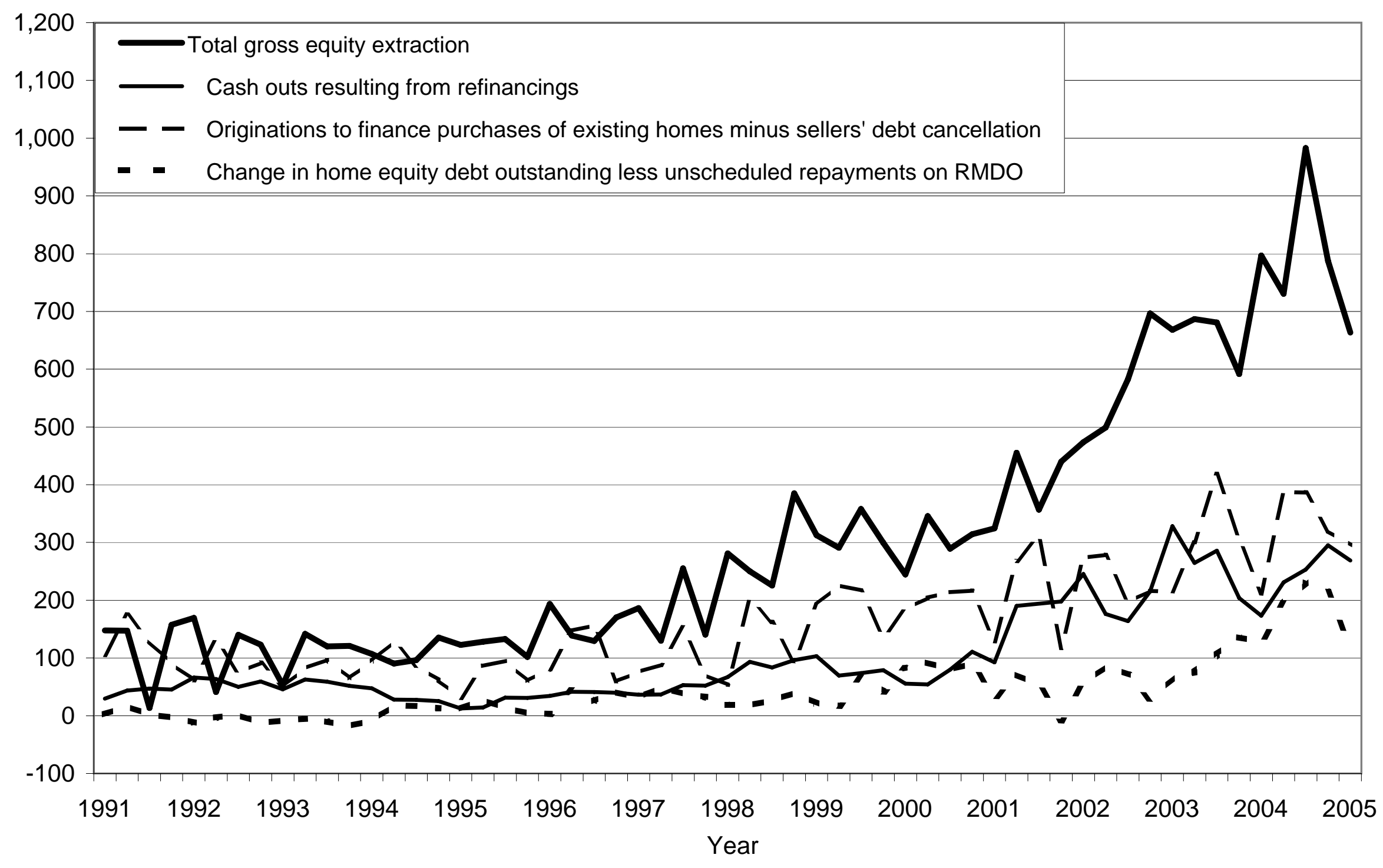


Figure 2

Equity Extraction

Billions of dollars

(1991:Q1-2005:Q1, seasonally adjusted annual rate)

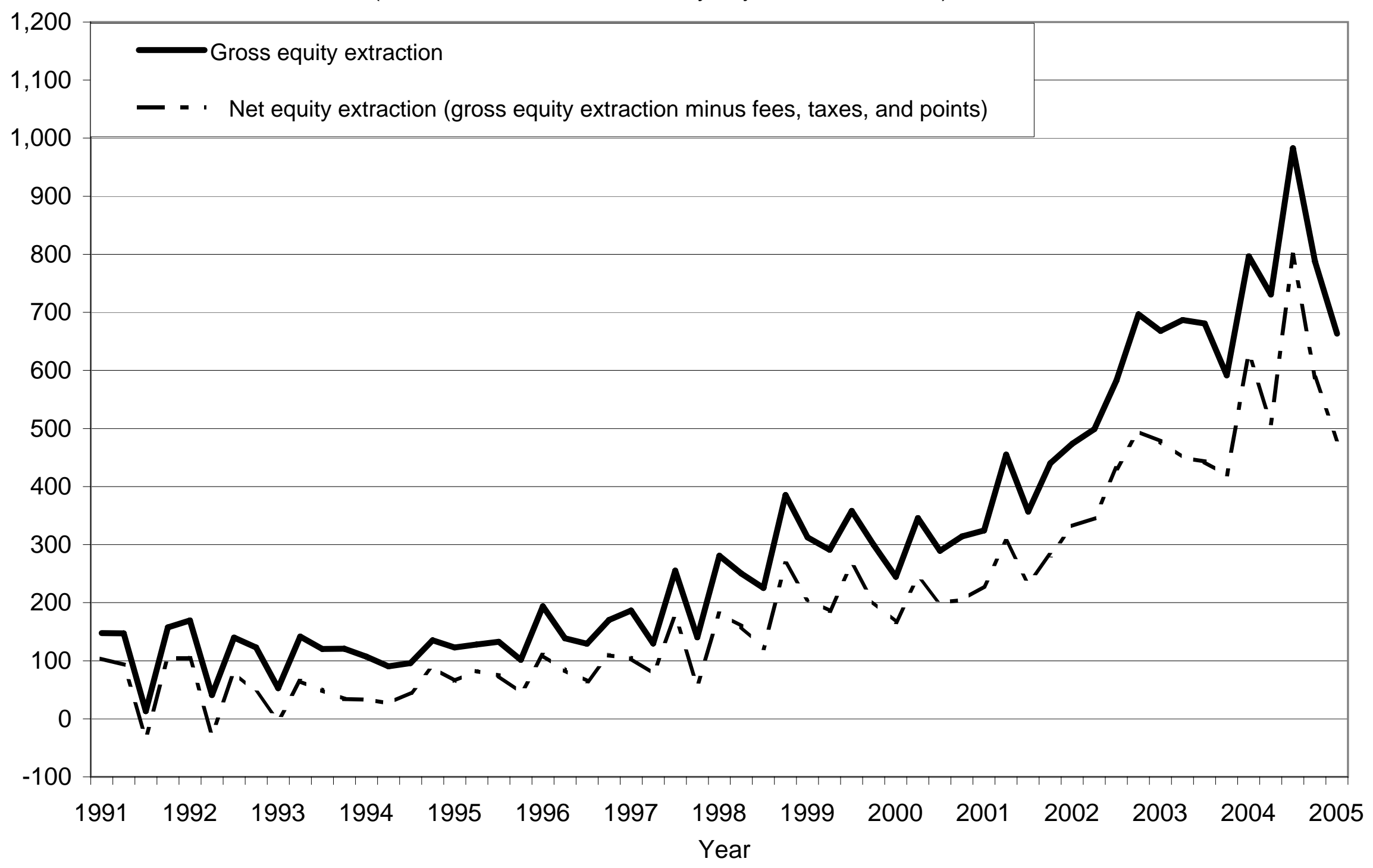


Figure 3

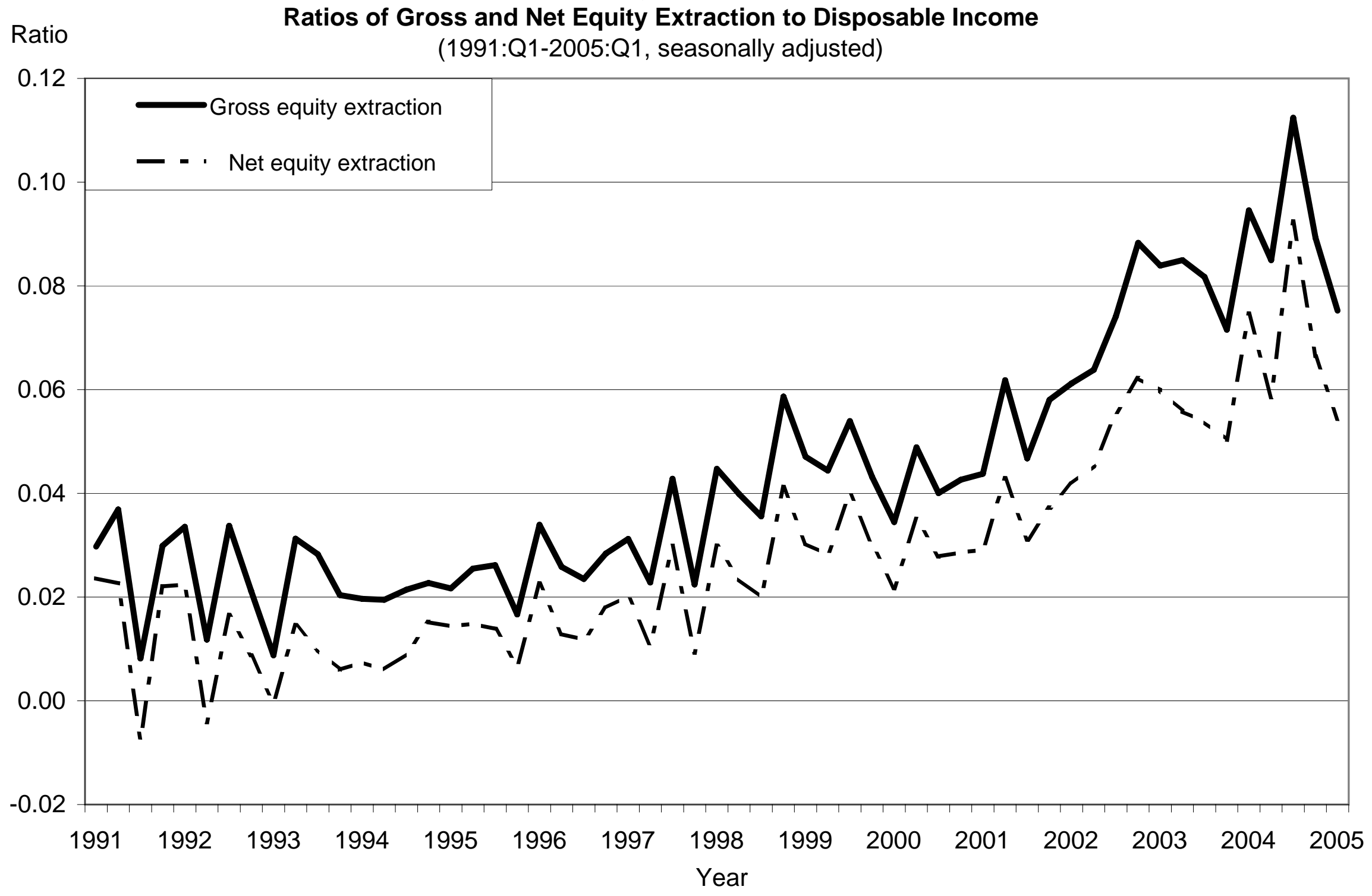


Figure 4

Repayment Rates on Regular Mortgage Debt

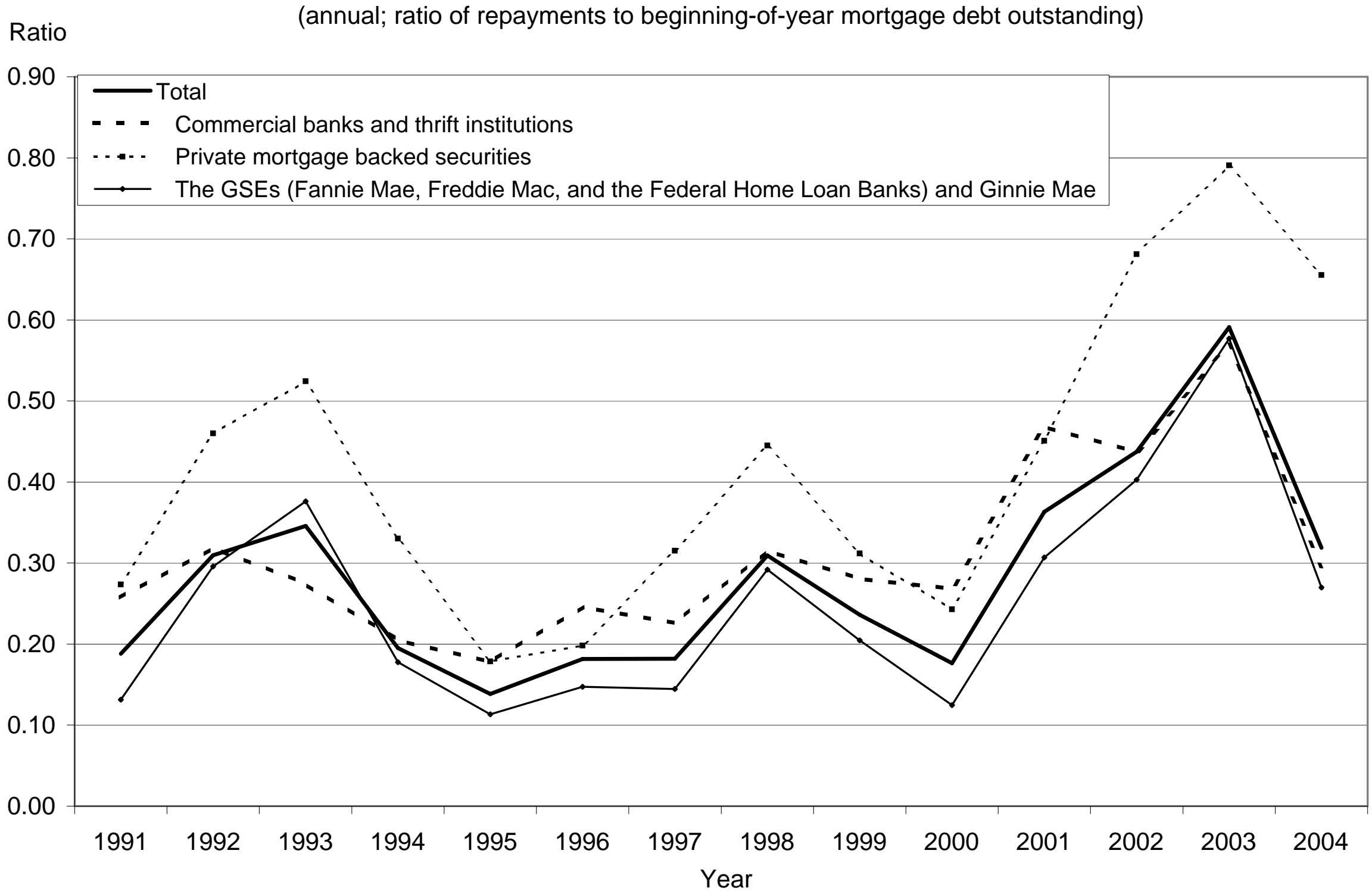


Figure 5

Regular Mortgages: Originations, Repayments, and the Change in Mortgage Debt

Outstanding

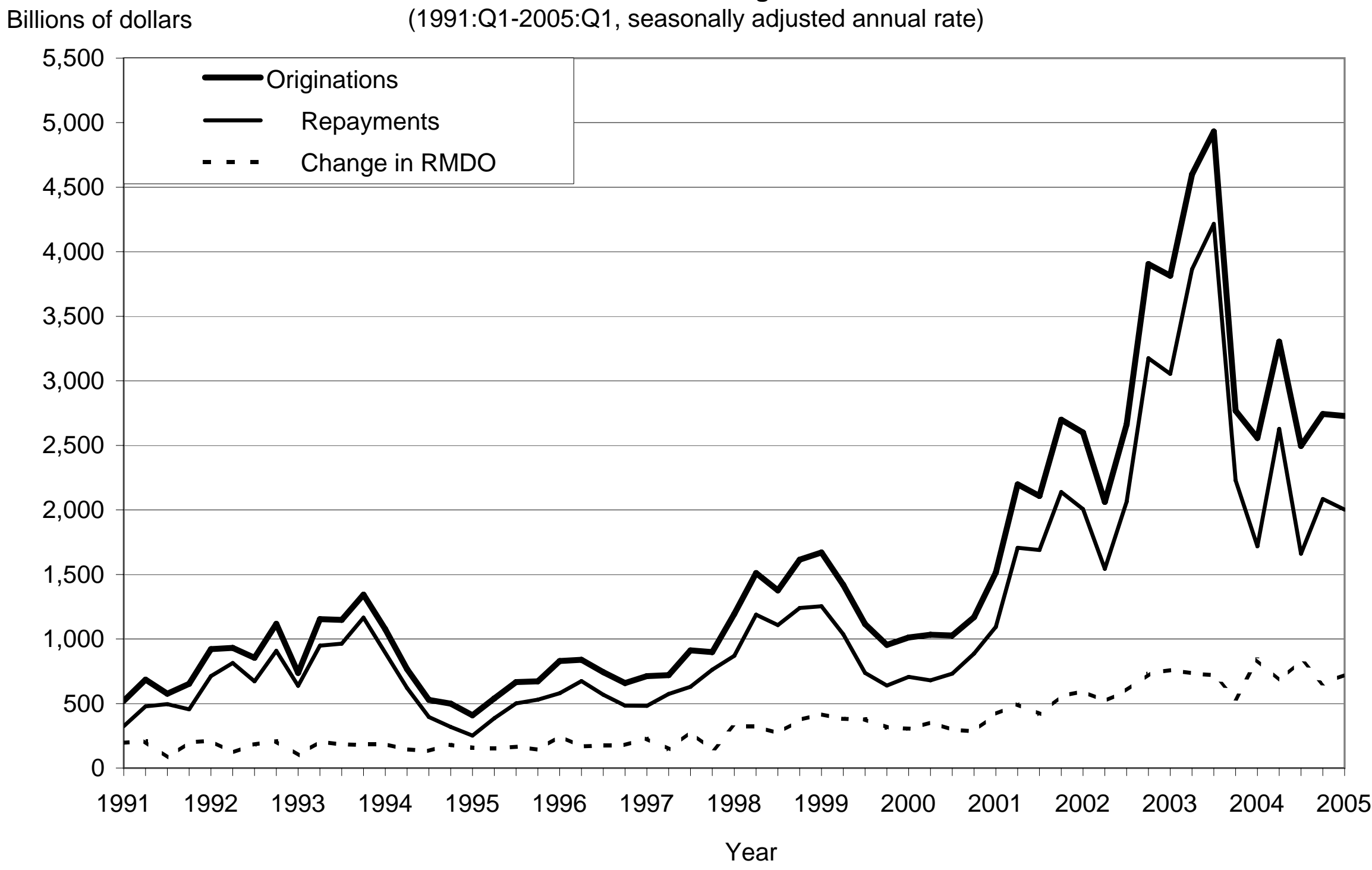


Figure 6

Regular Mortgage Originations to Purchase Homes and to Refinance Existing Loans

Billions of dollars

(1991:Q1-2005:Q1, seasonally adjusted annual rate)

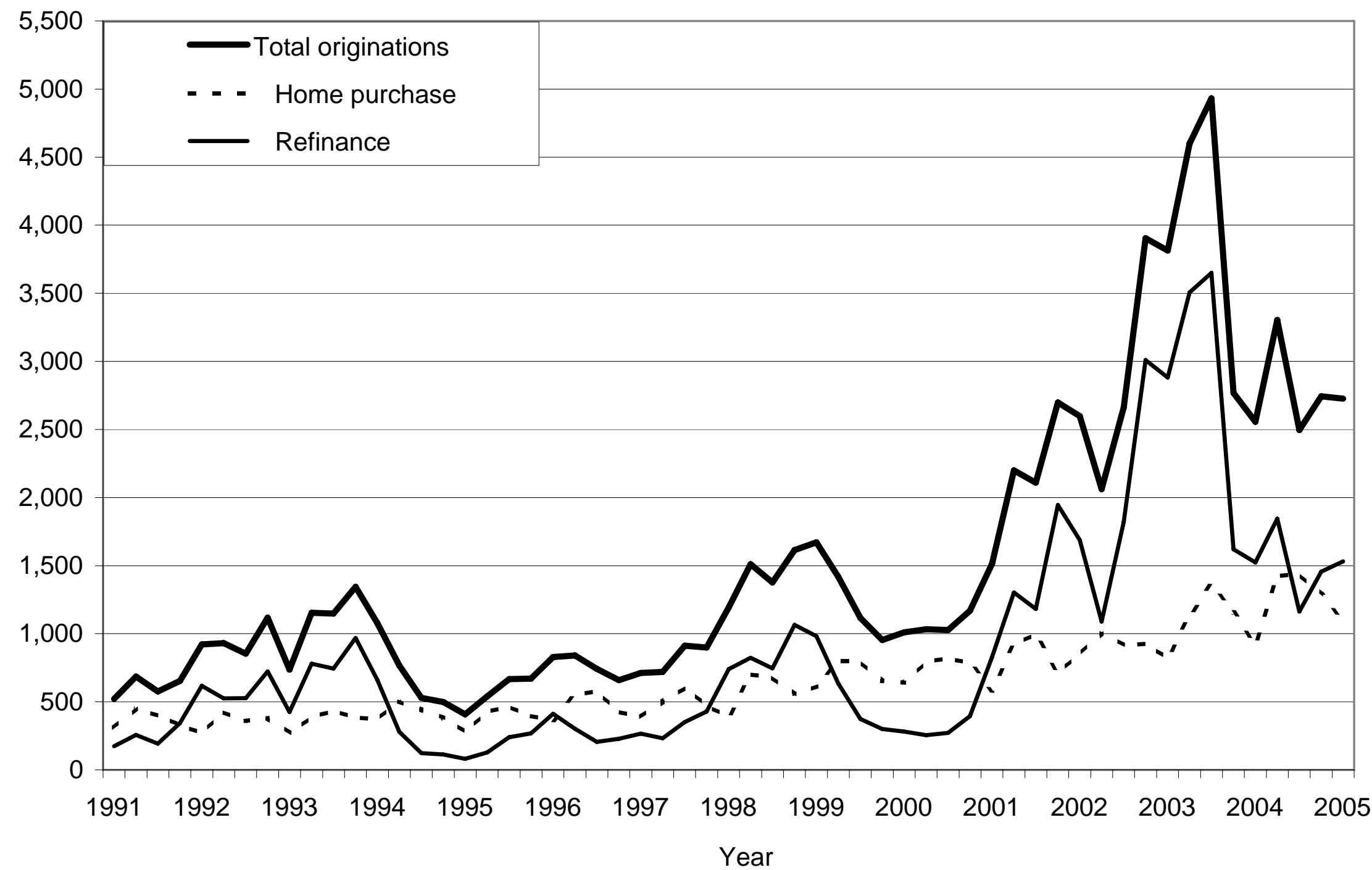


Figure 7

Billions of dollars

The Value of Homes Purchased

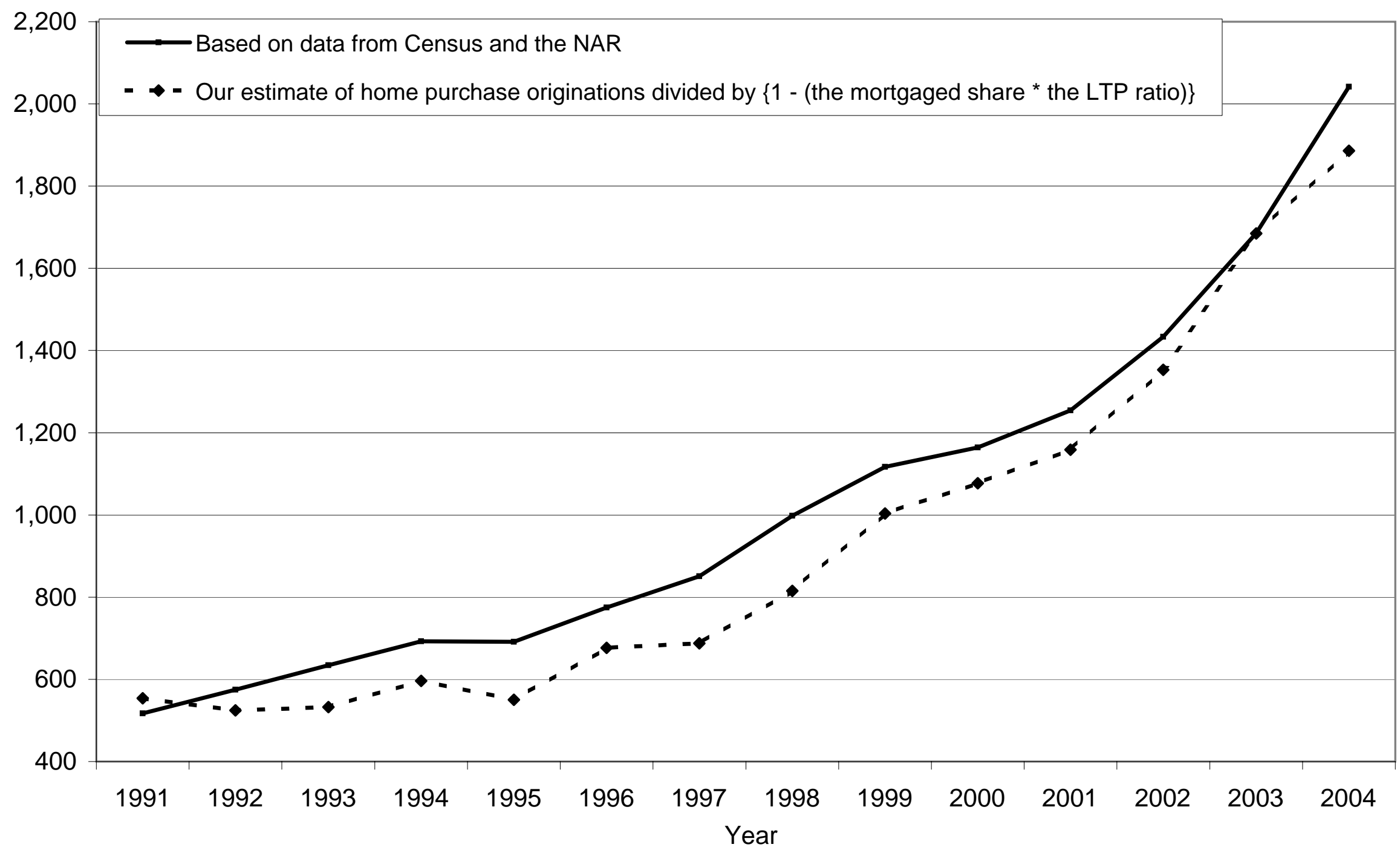


Figure 8

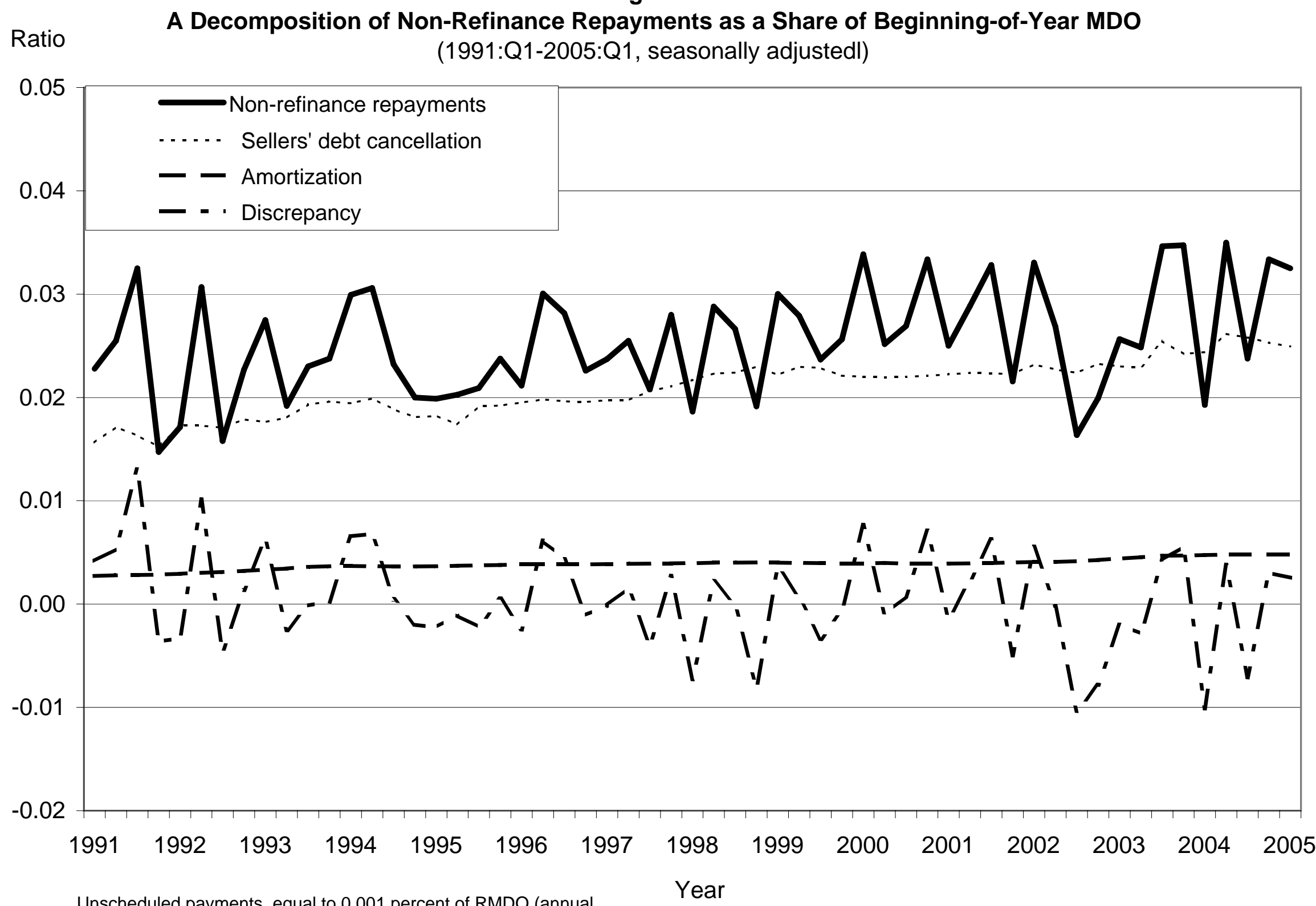

rate), are not shown separatley above. 
Table 1

Estimates of Mortgage Originations Derived from Mortgage Debt Outstanding and Repayments

(billions of dollars, except where noted; levels are end-of-year values, except where noted)

1991

1992

1993

1994

1995

1996

1997

1998

1999

\section{All Mortgagees}

(1) Net sales

(2) 1-4 mortgage debt outstanding, end of year (FOF)

(3) Construction loans

(4) Home equity loans

(5) Regular mortgages [(2)-(3)-(4)]

(6) Change $[(5)-(5)\{t-1\}]$

(8) Repayments $[(138)+(168)+(198)+(228)+(296)+(310)+(321)+(340)]$

(7) Repayment rate $[(8) /(5)\{t-1\}]$

(9) Total originations $[(6)+(8)]$

(10) Refinance originations $[(140)+(170)+(200)+(230)+(344)]$

(11) Refinance share $[(10) /(9)]$

(12) Purchase originations [(9)-(10)]

(13) GSE \& Ginnie Mae purchases / total originations [(286)/(9)]

(14) GSE, Ginnie Mae, \& private MBS purchases / originations [(326)/(9)]

(15) Value of homes purchased (source: Census and NAR)

(16) Value of homes purchased implied by purchase orig., the mort. share, and the wted. loan-to-price ratio $\left[\{(12) /(19)\}^{*}\left(1+0.78^{\star}\{(1-(18)) /(18)\}\right]\right.$ (sum of quart)

Price of non-mortgaged homes / price of mortgaged homes, fixed at 0.78

(17) Implied value of homes purchased / Census \& NAR value [(16)/(15)]

(18) Number of mortgaged purchases / total number of purchases

(19) Weighed loan-to-price ratio of homes purchased

(20) Mortgaged share * Ioan-to-price ratio [(18)*(19)]

(21) Gross equity extraction $[(6)+\{(4)-(4)\{t-1\}\}-(25)+(94)]$

(22) As a percent of disposable income

(23) Net equity extraction [(21)-(26)]

(24) As a percent of disposable income

(25) Memo: Originations to purchase new homes (see appendix E)

(26) Closing costs (see footnote 6 in main paper)

$\begin{array}{rrrrrrrrr}0.0 & 0.0 & 0.0 & 0.0 & 0.0 & 0.0 & 0.0 & 0.0 & 0.0 \\ 2,783.1 & 2,951.1 & 3,112.8 & 3,292.6 & 3,463.7 & 3,691.2 & 3,932.5 & 4,291.7 & 4,714.3 \\ 36.8 & 28.5 & 28.3 & 34.9 & 34.3 & 39.0 & 39.9 & 46.7 & 57.4 \\ 240.7 & 236.7 & 229.2 & 242.0 & 259.5 & 290.9 & 332.0 & 361.4 & 401.8 \\ \mathbf{2}, \mathbf{5 0 5 . 6} & \mathbf{2 , 6 8 5 . 9} & \mathbf{2 , 8 5 5 . 3} & \mathbf{3 , 0 1 5 . 8} & \mathbf{3 , 1 7 0 . 0} & \mathbf{3 , 3 6 1 . 2} & \mathbf{3 , 5 6 0 . 6} & \mathbf{3 , 8 8 3 . 6} & \mathbf{4 , 2 5 5 . 1} \\ 174.8 & 180.3 & 169.5 & 160.5 & 154.2 & 191.3 & 199.3 & \mathbf{3 2 3 . 1} & \mathbf{3 7 1 . 5} \\ & & & & & & & & \mathbf{9 1 6 . 7} \\ \mathbf{4 3 7 . 9} & \mathbf{7 7 6 . 0} & \mathbf{9 2 8 . 9} & \mathbf{5 5 7 . 0} & \mathbf{4 1 7 . 1} & \mathbf{5 7 6 . 0} & \mathbf{6 1 1 . 8} & \mathbf{1 , 1 0 1 . 7} & \mathbf{9 1 6 . 7} \\ \mathbf{0 . 1 8 7 9} & \mathbf{0 . 3 0 9 7} & \mathbf{0 . 3 4 5 8} & \mathbf{0 . 1 9 5 1} & \mathbf{0 . 1 3 8 3} & \mathbf{0 . 1 8 1 7} & \mathbf{0 . 1 8 2 0} & \mathbf{0 . 3 0 9 4} & \mathbf{0 . 2 3 6 0} \\ & & & & & & & & \\ \mathbf{6 1 2 . 7} & \mathbf{9 5 6 . 3} & \mathbf{1 , 0 9 8 . 3} & \mathbf{7 1 7 . 5} & \mathbf{5 7 1 . 3} & \mathbf{7 6 7 . 3} & \mathbf{8 1 1 . 2} & \mathbf{1 , 4 2 4 . 8} & \mathbf{1 , 2 8 8 . 2} \\ 241.8 & 598.4 & 729.1 & 293.5 & 179.8 & 286.9 & 319.6 & 843.5 & 572.5 \\ 0.3946 & 0.6258 & 0.6638 & 0.4091 & 0.3147 & 0.3740 & 0.3940 & 0.5921 & 0.4444 \\ 370.9 & 357.9 & 369.2 & 424.0 & 391.5 & 480.4 & 491.6 & 581.2 & 715.7 \\ & & & & & & & & \\ 0.4803 & 0.5441 & 0.5958 & 0.5434 & 0.5016 & 0.5028 & 0.4644 & 0.5373 & 0.5423 \\ 0.5745 & 0.6405 & 0.6902 & 0.6469 & 0.5838 & 0.5872 & 0.6056 & 0.6747 & 0.6545 \\ & & & & & & & & \\ 517.2 & 574.6 & 634.5 & 692.5 & 691.4 & 775.0 & 850.9 & 998.2 & 1,116.7 \\ & & & & & & & & \\ 554.0 & 524.6 & 532.6 & 596.5 & 550.3 & 676.9 & 687.4 & 815.0 & 1,003.2 \\ & & & & & & & & \\ 1.0712 & 0.9130 & 0.8394 & 0.8613 & 0.7959 & 0.8734 & 0.8079 & 0.8164 & 0.8983 \\ 0.8234 & 0.8267 & 0.8300 & 0.8333 & 0.8367 & 0.8400 & 0.8433 & 0.8467 & 0.8500 \\ 0.7812 & 0.7926 & 0.8035 & 0.8213 & 0.8200 & 0.8150 & 0.8189 & 0.8136 & 0.8116 \\ 0.6432 & 0.6553 & 0.6669 & 0.6844 & 0.6861 & 0.6846 & 0.6906 & 0.6889 & 0.6899 \\ 116.5 & 118.6 & 109.2 & 107.4 & 121.4 & 157.8 & 178.6 & \mathbf{2 8 6 . 1} & 316.0 \\ 2.61 & 2.50 & 2.22 & 2.08 & 2.25 & 2.77 & 2.98 & 4.47 & 4.72 \\ 67.0 & 49.5 & 34.0 & 48.0 & 67.3 & 93.0 & 106.6 & 182.7 & 216.2 \\ 1.50 & 1.04 & 0.69 & 0.93 & 1.24 & 1.63 & 1.78 & 2.86 & 3.23 \\ 91.9 & 89.2 & 91.1 & 108.5 & 95.9 & 114.8 & 115.5 & 125.1 & 159.9 \\ 49.6 & 69.1 & 75.2 & 59.4 & 54.1 & 64.9 & 72.0 & 103.5 & 99.8 \\ & & & & & & & & \end{array}$


Table 1

Estimates of Mortgage Originations Derived from Mortgage Debt Outstanding and Repayments

(billions of dollars, except where noted; levels are end-of-year values, except where noted)

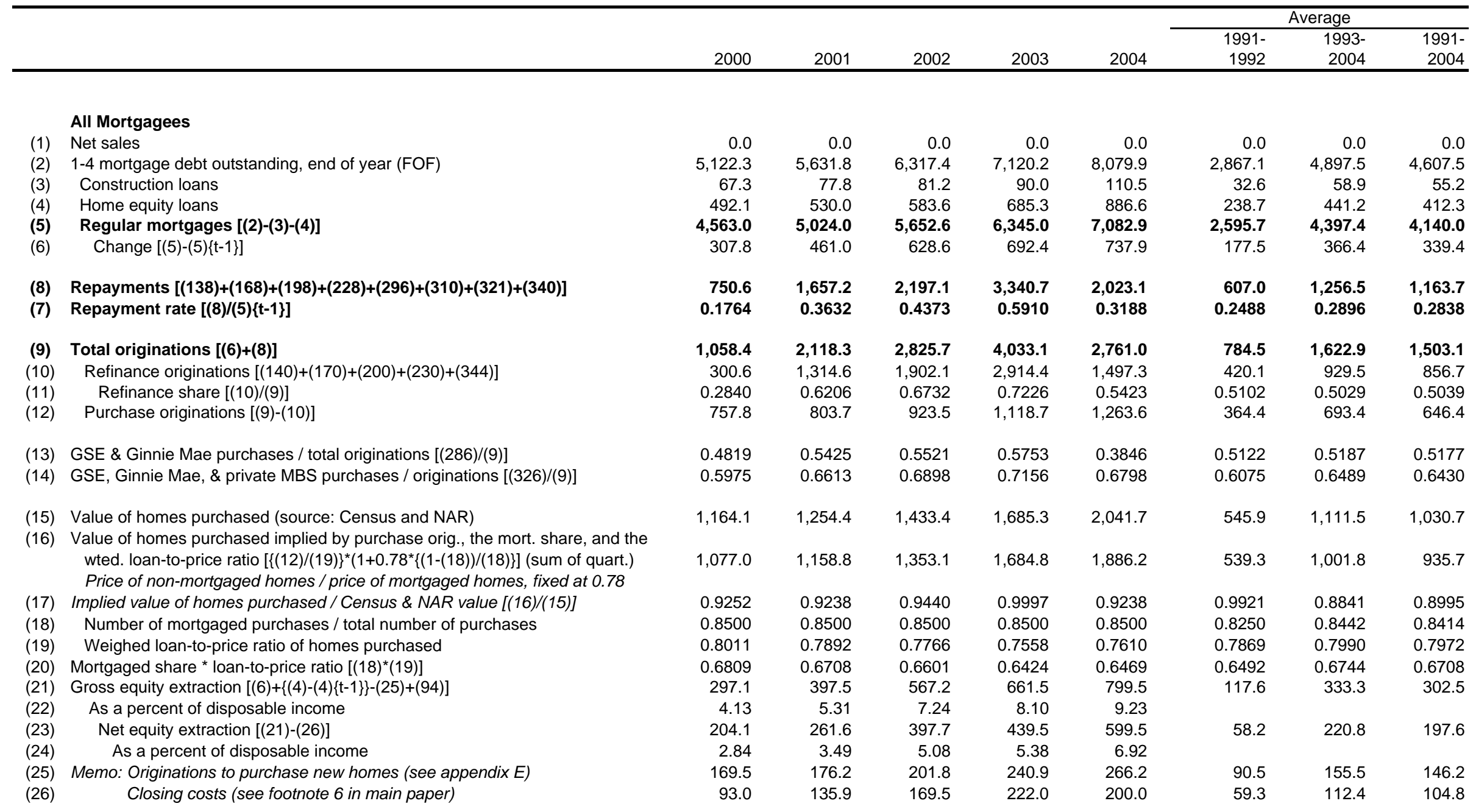


Table 1

Estimates of Mortgage Originations Derived from Mortgage Debt Outstanding and Repayments (billions of dollars, except where noted; levels are end-of-year values, except where noted)

\begin{tabular}{|c|c|c|c|c|c|c|c|c|c|c|}
\hline & & 1991 & 1992 & 1993 & 1994 & 1995 & 1996 & 1997 & 1998 & 1999 \\
\hline (27) & Our Originations [(9)] & 612.7 & 956.3 & $1,098.3$ & 717.5 & 571.3 & 767.3 & 811.2 & $1,424.8$ & $1,288.2$ \\
\hline (28) & Purchase [(12)] & 370.9 & 357.9 & 369.2 & 424.0 & 391.5 & 480.4 & 491.6 & 581.2 & 715.7 \\
\hline (29) & Refinance $[(10)]$ & 241.8 & 598.4 & 729.1 & 293.5 & 179.8 & 286.9 & 319.6 & 843.5 & 572.5 \\
\hline (30) & HMDA: total originations & 374.6 & 648.9 & 964.7 & 588.9 & 490.0 & 612.7 & 691.7 & $1,262.7$ & $1,040.1$ \\
\hline (31) & Purchase & 218.9 & 237.5 & 323.2 & 351.1 & 335.3 & 381.0 & 418.7 & 511.2 & 578.6 \\
\hline (32) & Refinance & 155.7 & 411.4 & 641.5 & 237.8 & 154.7 & 231.6 & 273.0 & 751.5 & 461.5 \\
\hline (33) & Ratio of HMDA to our originations $[(30) /(27)]$ & 0.6113 & 0.6785 & 0.8784 & 0.8208 & 0.8577 & 0.7985 & 0.8527 & 0.8863 & 0.8074 \\
\hline (34) & Home purchase originations $[(31) /(28)]$ & 0.5901 & 0.6636 & 0.8753 & 0.8282 & 0.8564 & 0.7932 & 0.8516 & 0.8795 & 0.8085 \\
\hline \multirow[t]{2}{*}{ (35) } & Refinance originations $[(32) /(29)]$ & 0.6439 & 0.6875 & 0.8799 & 0.8101 & 0.8606 & 0.8072 & 0.8543 & 0.8909 & 0.8061 \\
\hline & FHA Originations (purchase and refinance) & & & & & & & & & \\
\hline (36) & Source: HMDA & 41.0 & 38.6 & 106.1 & 53.8 & 48.1 & 63.5 & 70.8 & 98.7 & 99.3 \\
\hline (37) & As as share of total HMDA originations $[(36) /(30)]$ & 0.1095 & 0.0595 & 0.1100 & 0.0913 & 0.0981 & 0.1036 & 0.1024 & 0.0782 & 0.0954 \\
\hline (38) & Source: FHA & 47.0 & 48.3 & 79.1 & 91.6 & 45.3 & 71.6 & 74.2 & 103.2 & 122.3 \\
\hline \multirow[t]{2}{*}{ (39) } & HMDA coverage of FHA originations [(36)/(38)] & 0.8728 & 0.7994 & 1.3412 & 0.5869 & 1.0610 & 0.8866 & 0.9543 & 0.9567 & 0.8118 \\
\hline & VA Originations (purchase and refinance) & & & & & & & & & \\
\hline (41) & As as share of total HMDA originations $[(40) /(30)]$ & 0.0352 & 0.0335 & 0.0489 & 0.0513 & 0.0507 & 0.0452 & 0.0389 & 0.0381 & 0.0292 \\
\hline (42) & Source: VA & 15.9 & 25.1 & 41.6 & 49.5 & 23.9 & 33.4 & 26.9 & 42.6 & 49.6 \\
\hline \multirow[t]{2}{*}{ (43) } & HMDA coverage of VA originations [(40)/(42)] & 0.8268 & 0.8659 & 1.1328 & 0.6103 & 1.0378 & 0.8295 & 1.0018 & 1.1302 & 0.6120 \\
\hline & Comparison of Refinance Shares: & & & & & & & & & \\
\hline (44) & HMDA originations refinance share $[(32) /(30)]$ & 0.4156 & 0.6340 & 0.6650 & 0.4038 & 0.3157 & 0.3781 & 0.3947 & 0.5952 & 0.4437 \\
\hline (45) & Difference: Our implied refinance share - HMDA [(11)-(44)] & -0.0210 & -0.0082 & -0.0012 & 0.0053 & -0.0010 & -0.0041 & -0.0007 & -0.0031 & 0.0007 \\
\hline (46) & MBA mortgage applications refinance share (ave. of quarterly ratios) & 0.3409 & 0.4973 & 0.5274 & 0.2513 & 0.2268 & 0.2985 & 0.2978 & 0.5112 & 0.3565 \\
\hline (47) & Difference: Our implied refinance share - MBA [(11)-(46)] & 0.0537 & 0.1285 & 0.1365 & 0.1578 & 0.0879 & 0.0754 & 0.0962 & 0.0809 & 0.0879 \\
\hline (48) & Freddie Mac mortg. applications refi. share (ave. of quarterly ratios) & 0.4500 & 0.5150 & 0.5767 & 0.2692 & 0.2192 & 0.2967 & 0.3117 & 0.5192 & 0.3192 \\
\hline \multirow[t]{2}{*}{ (49) } & Difference: Our implied refinance share - Freddie Mac [(11)-(48)] & -0.0554 & 0.1108 & 0.0872 & 0.1400 & 0.0955 & 0.0773 & 0.0823 & 0.0729 & 0.1253 \\
\hline & FHA Originations Refinance Share (based on the $\$$ volume of orig.) & & & & & & & & & \\
\hline (50) & All FHA loans (source FHA) & 0.0562 & 0.2340 & 0.4512 & 0.2376 & 0.0951 & 0.1452 & 0.1385 & 0.3183 & 0.1941 \\
\hline (51) & FHA loans in HMDA & 0.0788 & 0.2677 & 0.5010 & 0.2441 & 0.1015 & 0.1387 & 0.1352 & 0.3278 & 0.1717 \\
\hline
\end{tabular}


Table 1

Estimates of Mortgage Originations Derived from Mortgage Debt Outstanding and Repayments (billions of dollars, except where noted; levels are end-of-year values, except where noted)

\begin{tabular}{|c|c|c|c|c|c|c|c|c|c|}
\hline & & & & & & & \multicolumn{3}{|c|}{ Average } \\
\hline & & 2000 & 2001 & 2002 & 2003 & 2004 & $\begin{array}{r}1991- \\
1992 \\
\end{array}$ & $\begin{array}{r}1993- \\
2004 \\
\end{array}$ & $\begin{array}{r}1991- \\
2004 \\
\end{array}$ \\
\hline (27) & Our Originations [(9)] & $1,058.4$ & $2,118.3$ & $2,825.7$ & $4,033.1$ & $2,761.0$ & 784.5 & $1,622.9$ & $1,503.1$ \\
\hline (28) & Purchase [(12)] & 757.8 & 803.7 & 923.5 & $1,118.7$ & $1,263.6$ & 364.4 & 693.4 & 646.4 \\
\hline (29) & Refinance $[(10)]$ & 300.6 & $1,314.6$ & $1,902.1$ & $2,914.4$ & $1,497.3$ & 420.1 & 929.5 & 856.7 \\
\hline (30) & HMDA: total originations & 846.1 & $1,760.7$ & $2,372.7$ & $3,301.1$ & $2,354.7$ & 511.7 & $1,357.2$ & $1,236.4$ \\
\hline (31) & Purchase & 606.8 & 665.3 & 764.7 & 901.1 & $1,077.2$ & 228.2 & 576.2 & 526.5 \\
\hline (32) & Refinance & 239.2 & $1,095.4$ & $1,608.0$ & $2,400.0$ & $1,277.4$ & 283.5 & 781.0 & 709.9 \\
\hline (33) & Ratio of HMDA to our originations $[(30) /(27)]$ & 0.7994 & 0.8312 & 0.8397 & 0.8185 & 0.8528 & 0.6449 & 0.8369 & 0.8095 \\
\hline (34) & Home purchase originations $[(31) /(28)]$ & 0.8007 & 0.8278 & 0.8280 & 0.8055 & 0.8525 & 0.6268 & 0.8339 & 0.8044 \\
\hline \multirow[t]{2}{*}{ (35) } & Refinance originations $[(32) /(29)]$ & 0.7959 & 0.8333 & 0.8454 & 0.8235 & 0.8532 & 0.6657 & 0.8384 & 0.8137 \\
\hline & FHA Originations (purchase and refinance) & & & & & & & & \\
\hline (36) & Source: HMDA & 87.1 & 138.9 & 127.3 & 143.4 & 75.6 & 39.8 & 92.7 & 85.2 \\
\hline (37) & As as share of total HMDA originations $[(36) /(30)]$ & 0.1030 & 0.0789 & 0.0536 & 0.0434 & 0.0321 & 0.0845 & 0.0825 & 0.0828 \\
\hline (38) & Source: FHA & 92.0 & 133.4 & 145.1 & 165.3 & 93.7 & 47.7 & 101.4 & 93.7 \\
\hline \multirow[t]{2}{*}{$(39)$} & HMDA coverage of FHA originations [(36)/(38)] & 0.9471 & 1.0415 & 0.8775 & 0.8675 & 0.8071 & 0.8361 & 0.9283 & 0.9151 \\
\hline & VA Originations (purchase and refinance) & & & & & & & & \\
\hline (40) & Source: HMDA & 20.6 & 37.4 & 39.9 & 59.0 & 29.7 & 17.5 & 35.2 & 32.6 \\
\hline (41) & As as share of total HMDA originations $[(40) /(30)]$ & 0.0243 & 0.0212 & 0.0168 & 0.0179 & 0.0126 & 0.0 & 0.0 & 0.0 \\
\hline (42) & Source: VA & 22.2 & 35.4 & 41.9 & 66.1 & 35.3 & 20.5 & 39.0 & 36.4 \\
\hline \multirow[t]{2}{*}{$(43)$} & HMDA coverage of VA originations $[(40) /(42)]$ & 0.9266 & 1.0553 & 0.9519 & 0.8925 & 0.8401 & 0.8464 & 0.9184 & 0.9081 \\
\hline & Comparison of Refinance Shares: & & & & 202.4557 & & & & \\
\hline (44) & HMDA originations refinance share $[(32) /(30)]$ & 0.2828 & 0.6221 & 0.6777 & 0.7270 & 0.5425 & 0.5248 & 0.5040 & 0.5070 \\
\hline (45) & Difference: Our implied refinance share - HMDA [(11)-(44)] & 0.0012 & -0.0015 & -0.0045 & -0.0044 & -0.0002 & -0.0146 & -0.0011 & -0.0031 \\
\hline (46) & MBA mortgage applications refinance share (ave. of quarterly ratios) & 0.2042 & 0.5503 & 0.5696 & 0.6426 & 0.4372 & 0.4191 & 0.4061 & 0.4080 \\
\hline (47) & Difference: Our implied refinance share - MBA [(11)-(46)] & 0.0798 & 0.0703 & 0.1035 & 0.0800 & 0.1052 & 0.0911 & 0.0968 & 0.0960 \\
\hline (48) & Freddie Mac mortg. applications refi. share (ave. of quarterly ratios) & 0.2125 & 0.5675 & 0.5883 & 0.6475 & 0.4400 & 0.4825 & 0.4140 & 0.4238 \\
\hline \multirow[t]{3}{*}{ (49) } & Difference: Our implied refinance share - Freddie Mac [(11)-(48)] & 0.0715 & 0.0531 & 0.0848 & 0.0751 & 0.1023 & 0.0277 & 0.0889 & 0.0802 \\
\hline & & & & & & & & $1999-2003$ & \\
\hline & FHA Originations Refinance Share (based on the $\$$ volume of orig.) & & & & & & & & \\
\hline (50) & All FHA loans (source FHA) & 0.0792 & 0.3337 & 0.3430 & 0.4696 & 0.3269 & 0.1451 & 0.2610 & 0.2445 \\
\hline (51) & FHA loans in HMDA & 0.0736 & 0.3425 & 0.3559 & 0.4903 & 0.3198 & 0.1732 & 0.2668 & 0.2535 \\
\hline
\end{tabular}


Table 1

Estimates of Mortgage Originations Derived from Mortgage Debt Outstanding and Repayments (billions of dollars, except where noted; levels are end-of-year values, except where noted)

\begin{tabular}{|c|c|c|c|c|c|c|c|c|c|c|}
\hline & & 1991 & 1992 & 1993 & 1994 & 1995 & 1996 & 1997 & 1998 & 1999 \\
\hline (52) & Repayments $[(8)]$ & 437.9 & 776.0 & 928.9 & 557.0 & 417.1 & 576.0 & 611.8 & $1,101.7$ & 916.7 \\
\hline (53) & Excluding mortgage companies and "other" mortgagees & 371.9 & 670.7 & 821.6 & 498.9 & 377.8 & 526.2 & 564.1 & $1,026.0$ & 858.6 \\
\hline (54) & Commercial banks and thrift institutions & 208.0 & 247.3 & 210.6 & 165.0 & 152.2 & 222.7 & 214.0 & 315.4 & 295.6 \\
\hline (55) & Commercial banks [(138)] & 78.9 & 91.2 & 78.3 & 73.0 & 67.7 & 133.7 & 106.5 & 148.7 & 157.1 \\
\hline (56) & Thrifts regulated by OTS [(168)] & 113.2 & 132.3 & 108.6 & 73.5 & 68.7 & 71.8 & 89.2 & 139.1 & 115.5 \\
\hline (57) & Savings banks $[(198)]$ & 15.9 & 23.9 & 23.7 & 18.5 & 15.8 & 17.2 & 18.3 & 27.7 & 23.0 \\
\hline (58) & Credit unions $[(228)]$ & 3.8 & 8.1 & 8.3 & 5.6 & 5.3 & 6.3 & 7.4 & 12.6 & 12.1 \\
\hline (59) & GSEs and Ginnie Mae [(296)] & 145.0 & 370.3 & 526.7 & 271.3 & 186.3 & 257.0 & 271.0 & 577.6 & 443.1 \\
\hline (61) & Private MBS $[(310)]$ & 15.1 & 45.0 & 76.0 & 57.0 & 33.9 & 40.2 & 71.7 & 120.3 & 107.8 \\
\hline (62) & Other mortgagees that do not originate loans [(321)] & 54.2 & 88.0 & 93.5 & 49.9 & 34.2 & 42.5 & 40.5 & 69.1 & 52.1 \\
\hline (63) & Mortgage companies $[(340)]$ & 11.9 & 17.4 & 13.7 & 8.3 & 5.1 & 7.3 & 7.3 & 6.6 & 6.0 \\
\hline (64) & Level of regular mortgage debt $[(5)]$ & $2,505.6$ & $2,685.9$ & $2,855.3$ & $3,015.8$ & $3,170.0$ & $3,361.2$ & $3,560.6$ & $3,883.6$ & $4,255.1$ \\
\hline (65) & Excluding mortgage companies and "other" mortgagees & $2,157.3$ & $2,346.3$ & $2,541.1$ & $2,731.7$ & $2,897.8$ & $3,100.8$ & $3,307.2$ & $3,634.5$ & $4,006.4$ \\
\hline (66) & Commercial banks and thrift institutions & 775.9 & 767.3 & 804.6 & 855.2 & 905.7 & 948.5 & $1,000.0$ & $1,054.1$ & $1,124.3$ \\
\hline (67) & Commercial banks [(135)] & 308.3 & 341.8 & 395.8 & 439.5 & 487.0 & 502.7 & 545.7 & 589.0 & 650.5 \\
\hline (68) & Thrifts regulated by OTS [(165)] & 380.7 & 341.3 & 323.0 & 326.8 & 323.3 & 345.4 & 354.6 & 363.0 & 368.8 \\
\hline (71) & GSEs and Ginnie Mae [(293)] & $1,252.3$ & $1,400.7$ & $1,527.2$ & $1,645.4$ & $1,745.6$ & $1,874.3$ & $1,979.8$ & $2,167.4$ & $2,421.8$ \\
\hline (72) & GSEs $[(251)+(261)+(282)]$ & 839.3 & 992.8 & $1,124.5$ & $1,206.0$ & $1,285.5$ & $1,381.5$ & $1,457.3$ & $1,645.3$ & $1,856.9$ \\
\hline (73) & Private MBS [308)] & 97.7 & 145.0 & 172.7 & 189.9 & 202.9 & 227.5 & 270.3 & 345.9 & 382.7 \\
\hline (74) & Other mortgagees that do not originate loans [(318)] & 295.2 & 286.8 & 262.0 & 255.8 & 248.4 & 228.4 & 231.5 & 227.3 & 226.9 \\
\hline (75) & Mortgage companies $[(337)]$ & 53.1 & 52.7 & 52.2 & 28.3 & 23.8 & 32.0 & 21.8 & 21.8 & 21.8 \\
\hline (76) & Repayment rate $[(52) /(64)\{t-1\}]$ & 0.1879 & 0.3097 & 0.3458 & 0.1951 & 0.1383 & 0.1817 & 0.1820 & 0.3094 & 0.2360 \\
\hline (77) & Excluding mort. companies and "other" mortgagees $[(53) /(65)\{t-1\}]$ & 0.1862 & 0.3109 & 0.3502 & 0.1963 & 0.1383 & 0.1816 & 0.1819 & 0.3102 & 0.2362 \\
\hline (78) & Commercial banks and thrift institutions $[(54) /(66)\{t-1\}]$ & 0.2575 & 0.3188 & 0.2744 & 0.2050 & 0.1780 & 0.2459 & 0.2256 & 0.3154 & 0.2804 \\
\hline (79) & Commercial banks $[(55) /(67)\{t-1\}]$ & 0.2765 & 0.2957 & 0.2292 & 0.1844 & 0.1541 & 0.2747 & 0.2119 & 0.2725 & 0.2667 \\
\hline (80) & Thrifts regulated by OTS $[(56) /(68)\{t-1\}]$ & 0.2589 & 0.3474 & 0.3181 & 0.2274 & 0.2101 & 0.2219 & 0.2582 & 0.3922 & 0.3183 \\
\hline (81) & Savings banks $[(57) /(69)\{t-1\}]$ & 0.1869 & 0.2754 & 0.2809 & 0.2156 & 0.1782 & 0.1806 & 0.1819 & 0.2774 & 0.2253 \\
\hline (82) & Credit unions $[(58) /(70)\{t-1\}]$ & 0.1275 & 0.2570 & 0.2505 & 0.1530 & 0.1294 & 0.1439 & 0.1459 & 0.2212 & 0.1809 \\
\hline (83) & GSEs and Ginnie Mae $[(59) /(71)\{t-1\}]$ & 0.1313 & 0.2957 & 0.3760 & 0.1777 & 0.1132 & 0.1472 & 0.1446 & 0.2917 & 0.2044 \\
\hline (84) & GSEs $[(60) /(72)]$ & 0.1484 & 0.3392 & 0.3887 & 0.1769 & 0.1131 & 0.1488 & 0.1441 & 0.2951 & 0.2050 \\
\hline (85) & Private MBS $[(61) /(73)\{\mathrm{t}-1\}]$ & 0.2736 & 0.4601 & 0.5243 & 0.3300 & 0.1786 & 0.1981 & 0.3152 & 0.4451 & 0.3116 \\
\hline (86) & Other mortgagees that do not originate loans $[(62) /(74)\{t-1\}]$ & 0.1853 & 0.2982 & 0.3259 & 0.1904 & 0.1337 & 0.1710 & 0.1773 & 0.2984 & 0.2293 \\
\hline (87) & Mortgage companies $[(63) /(75)\{t-1\}]$ & 0.2869 & 0.3271 & 0.2607 & 0.1591 & 0.1804 & 0.3090 & 0.2270 & 0.3039 & 0.2732 \\
\hline (88) & Memo: commercial banks, thrift institutions, and private MBS & 0.2586 & 0.3346 & 0.3141 & 0.2271 & 0.1781 & 0.2372 & 0.2429 & 0.3430 & 0.2881 \\
\hline
\end{tabular}


Table 1

Estimates of Mortgage Originations Derived from Mortgage Debt Outstanding and Repayments

(billions of dollars, except where noted; levels are end-of-year values, except where noted)

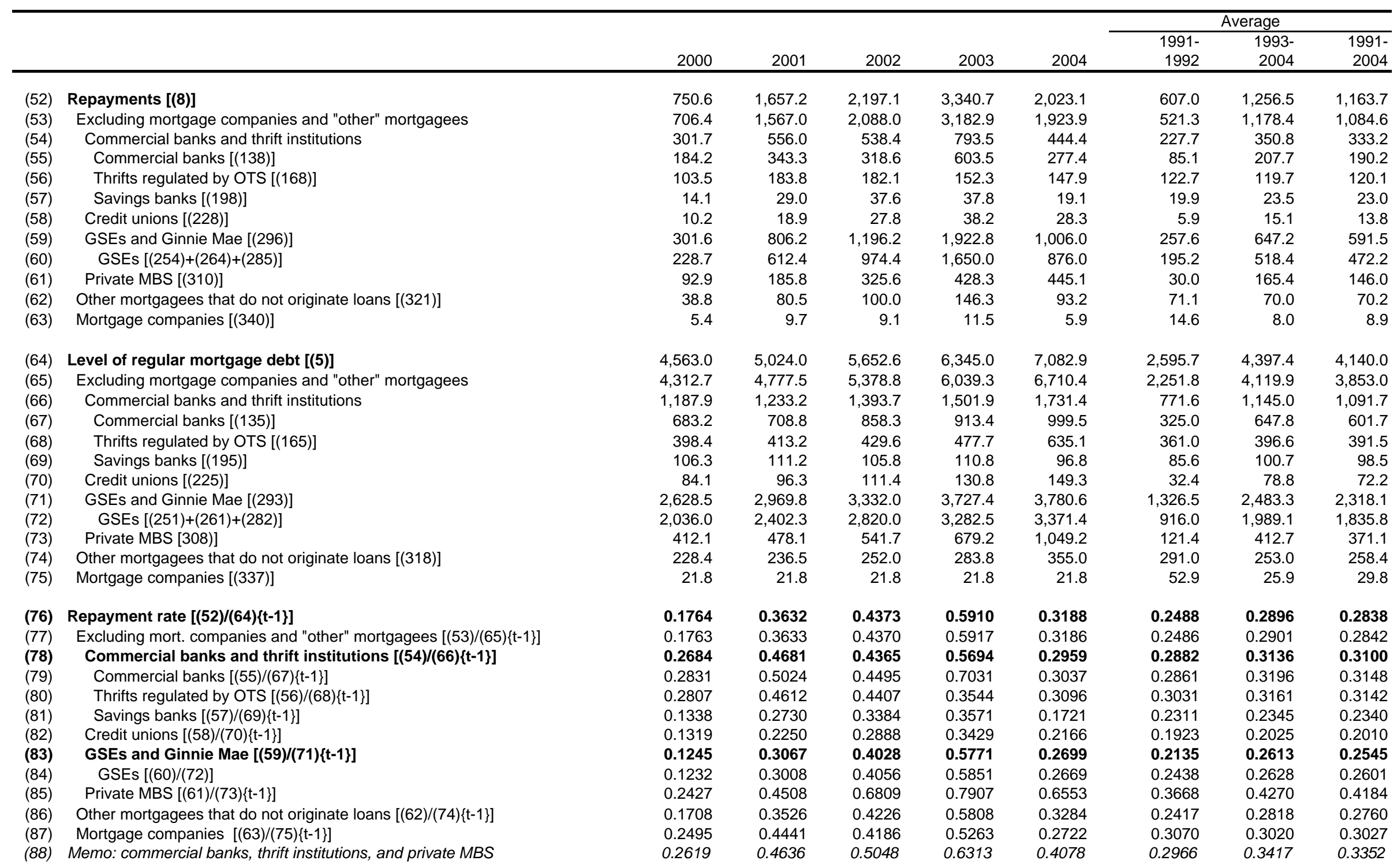


Table 1

Estimates of Mortgage Originations Derived from Mortgage Debt Outstanding and Repayments

(billions of dollars, except where noted; levels are end-of-year values, except where noted)

1991

1992

1993

1994

1995

1996

1997

1998

1999

\section{Decompostion of Repayments}

(89) Total repayments [(8)]

(90) Repayments resulting from refinance originations [(10)-(124)]

(91) As a share of beginning-of-year MDO [(90)/(5)\{t-1\}]

(92) Repayments excluding refinancings [(89)-(90)]

(93) As a share of beginning-of-year MDO [(92)/(5)\{t-1\}]

\begin{tabular}{|c|c|c|c|c|c|c|c|c|}
\hline 437.9 & 776.0 & 928.9 & 557.0 & 417.1 & 576.0 & 611.8 & $1,101.7$ & 916.7 \\
\hline 209.7 & 555.1 & 674.0 & 255.7 & 155.6 & 243.9 & 272.9 & 756.9 & 482.4 \\
\hline 0.0900 & 0.2216 & 0.2509 & 0.0896 & 0.0516 & 0.0769 & 0.0812 & 0.2126 & 0.1242 \\
\hline 228.2 & 220.9 & 254.8 & 301.3 & 261.5 & 332.2 & 338.9 & 344.8 & 434.3 \\
\hline 0.0979 & 0.0882 & 0.0949 & 0.1055 & 0.0867 & 0.1048 & 0.1008 & 0.0968 & 0.1118 \\
\hline 26.8 & 31.6 & 38.3 & 42.6 & 45.7 & 49.9 & 53.6 & 58.8 & 64.0 \\
\hline 153.7 & 177.7 & 203.4 & 222.0 & 228.1 & 256.0 & 281.0 & 331.1 & 366.2 \\
\hline 11.0 & 12.4 & 12.8 & 15.2 & 15.0 & 15.1 & 18.0 & 20.5 & 20.7 \\
\hline 142.7 & 165.4 & 190.6 & 206.9 & 213.1 & 240.9 & 263.0 & 310.6 & 345.5 \\
\hline 2.4 & 2.6 & 2.7 & 2.9 & 3.1 & 3.2 & 3.4 & 3.7 & 4.0 \\
\hline 45.3 & 9.1 & 10.4 & 33.8 & -15.3 & 23.0 & 0.8 & -48.8 & 0.2 \\
\hline 0.0115 & 0.0126 & 0.0143 & 0.0149 & 0.0152 & 0.0157 & 0.0160 & 0.0165 & 0.0165 \\
\hline 0.0659 & 0.0709 & 0.0757 & 0.0778 & 0.0756 & 0.0808 & 0.0836 & 0.0930 & 0.0943 \\
\hline 0.0047 & 0.0049 & 0.0048 & 0.0053 & 0.0050 & 0.0048 & 0.0054 & 0.0057 & 0.0053 \\
\hline 0.0612 & 0.0660 & 0.0710 & 0.0724 & 0.0707 & 0.0760 & 0.0782 & 0.0872 & 0.0890 \\
\hline 0.0010 & 0.0010 & 0.0010 & 0.0010 & 0.0010 & 0.0010 & 0.0010 & 0.0010 & 0.0010 \\
\hline 0.0194 & 0.0036 & 0.0039 & 0.0118 & -0.0051 & 0.0072 & 0.0002 & -0.0137 & 0.0000 \\
\hline 9.155 & 8.398 & 7.250 & 8.478 & 7.716 & 7.943 & 7.528 & 6.891 & 7.581 \\
\hline 9.664 & 9.137 & 8.531 & 8.094 & 8.076 & 7.937 & 7.885 & 7.630 & 7.373 \\
\hline 23.734 & 23.619 & 23.222 & 23.423 & 23.264 & 23.124 & 23.049 & 23.179 & 23.675 \\
\hline 258.2 & 266.2 & 271.8 & 278.3 & 293.7 & 307.3 & 324.4 & 338.8 & 360.7 \\
\hline 231.4 & 234.6 & 233.4 & 235.7 & 247.9 & 257.4 & 270.8 & 280.0 & 296.7 \\
\hline 26.8 & 31.6 & 38.3 & 42.6 & 45.7 & 49.9 & 53.6 & 58.8 & 64.0 \\
\hline 82.3 & 85.9 & 89.6 & 94.5 & 97.0 & 101.4 & 107.5 & 112.1 & 117.4 \\
\hline 40.7 & 39.2 & 37.5 & 36.7 & 36.3 & 37.7 & 39.8 & 43.5 & 47.1 \\
\hline 97.9 & 100.9 & 104.0 & 108.6 & 110.9 & 115.9 & 122.8 & 128.8 & 135.4 \\
\hline 84.8 & 87.4 & 90.1 & 94.0 & 96.0 & 100.4 & 106.3 & 111.5 & 117.3 \\
\hline 506.2 & 555.3 & 558.3 & 632.0 & 611.5 & 589.7 & 663.3 & 719.0 & 690.6 \\
\hline 151.9 & 166.6 & 167.5 & 189.6 & 183.4 & 176.9 & 199.0 & 215.7 & 207.2 \\
\hline 12.9 & 14.6 & 15.1 & 17.8 & 17.6 & 17.8 & 21.2 & 24.1 & 24.3 \\
\hline na & 4.2 & 4.3 & 4.2 & 3.8 & 4.3 & 5.2 & 5.8 & 1.3 \\
\hline & 0.3402 & 0.3391 & 0.2771 & 0.2550 & 0.2858 & 0.2865 & 0.2831 & 0.0633 \\
\hline 1.7 & 1.7 & 1.7 & 1.6 & 1.5 & 1.6 & 2.1 & 2.4 & 1.8 \\
\hline 0.1561 & 0.1406 & 0.1310 & 0.1067 & 0.0989 & 0.1080 & 0.1167 & 0.1184 & 0.0870 \\
\hline 0.1430 & 0.0746 & 0.0839 & 0.1753 & 0.1578 & 0.1667 & 0.1596 & 0.1099 & 0.1843 \\
\hline 32.1 & 43.3 & 55.1 & 37.8 & 24.2 & 43.1 & 46.7 & 86.6 & 90.1 \\
\hline 0.1327 & 0.0724 & 0.0756 & 0.1289 & 0.1346 & 0.1501 & 0.1460 & 0.1027 & 0.1574 \\
\hline 5.0 & 24.9 & 79.2 & 27.0 & 10.2 & 18.4 & 19.8 & 70.8 & 36.3 \\
\hline
\end{tabular}

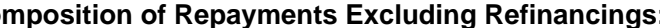

(94) Amortization [(111)]

(95) Sellers' debt cancellation

(96) Resulting from foreclosure sales [(118)]

(97) Resulting from non-foreclosure sales

(98) Unscheduled repayments $(0.001 * \mathrm{MDO}\{\mathrm{t}-1\})$

(99) Discrepancy [(92)-(94)-(95)-(98)]

Non-Refinance Repayments as a Share of Beginning-of-Year MDO:

(100) Amortization $[(94) /(5)\{t-1\})]$

(101) Sellers' debt cancellation $[(95) /(5)\{t-1\})]$

(102) Resulting from foreclosure sales [(131)]

(103) Resulting from non-foreclosure sales

(104) Unscheduled payments $(0.001 * \mathrm{MDO}\{\mathrm{t}-1\})$

(105) Discrepancy $[(99) /(5)\{t-1\})]$

Memo:

\section{Calculation of Amortization:}

(106) Thirty-year fixed mortgage rate (contract rate, average of quarterly)

(107) Weighted-average coupon rate (our estimate, average of quarterly)

(108) Average term to maturity of outstanding mortgages (years, Citigroup)

(109) Total scheduled payments [fct((107),(108),(5\{t-1\}))] billions of dollars

(110) Interest $\left[\left(0.01^{*}(107)^{\star}(5)\{t-1\} / 4\right]\right.$ (sum of quarterly)

(111) Principal (amortization) [(109)-(110)]

Foreclosures (see appendix $\mathrm{E}$ for more information):

(112) Average acquisition price of all units sold in period (thou. \$, our estimate)

(113) Average realized capital gain, all units sold in period (our estimate)

(114) Average acquisition price, foreclosed units $\left[0.85^{*}(112)+(113)^{\star}(1-.6166)\right]$

(115) Debt cancellation per foreclosure sale [(114)*0.9*.96219]

(116) Exits from foreclosure (thousands of units, our estimate based on MBA data)

(117) Number of foreclosed units assuming a cure rate of $70 \%\left[(116)^{\star} 0.3\right]$

(118) Chargeoffs, foreclosed units (mil. \$) $\left[0.001 *(115)^{\star}(117)\right]$

(119) Chargeoffs of FHA loans (billions of dollars)

(120) As a share of total foreclosure chargeoffs [(119)/(96)]

(121) Chargeoffs of VA loans (billions of dollars)

(122) As a share of total foreclosure chargeoffs [(121)/(96)]

Separating Refinance Originations into the Gross Cash Out and Repayment of the Retired Loan

(123) Gross cash out share, conventional loans (source: Freddie Mac, ave. quart.)

(124) Gross cash out (sum of quarterly) $\left[\left\{(123)^{*}((10)-(126))\right\}+\left\{(126)^{*} 0.03\right\}\right]$

(125) Gross cash out / refinance originations [(124)/(10)]

(126) Memo: FHA and VA refinance originations 
Table 1

Estimates of Mortgage Originations Derived from Mortgage Debt Outstanding and Repayments

(billions of dollars, except where noted; levels are end-of-year values, except where noted)

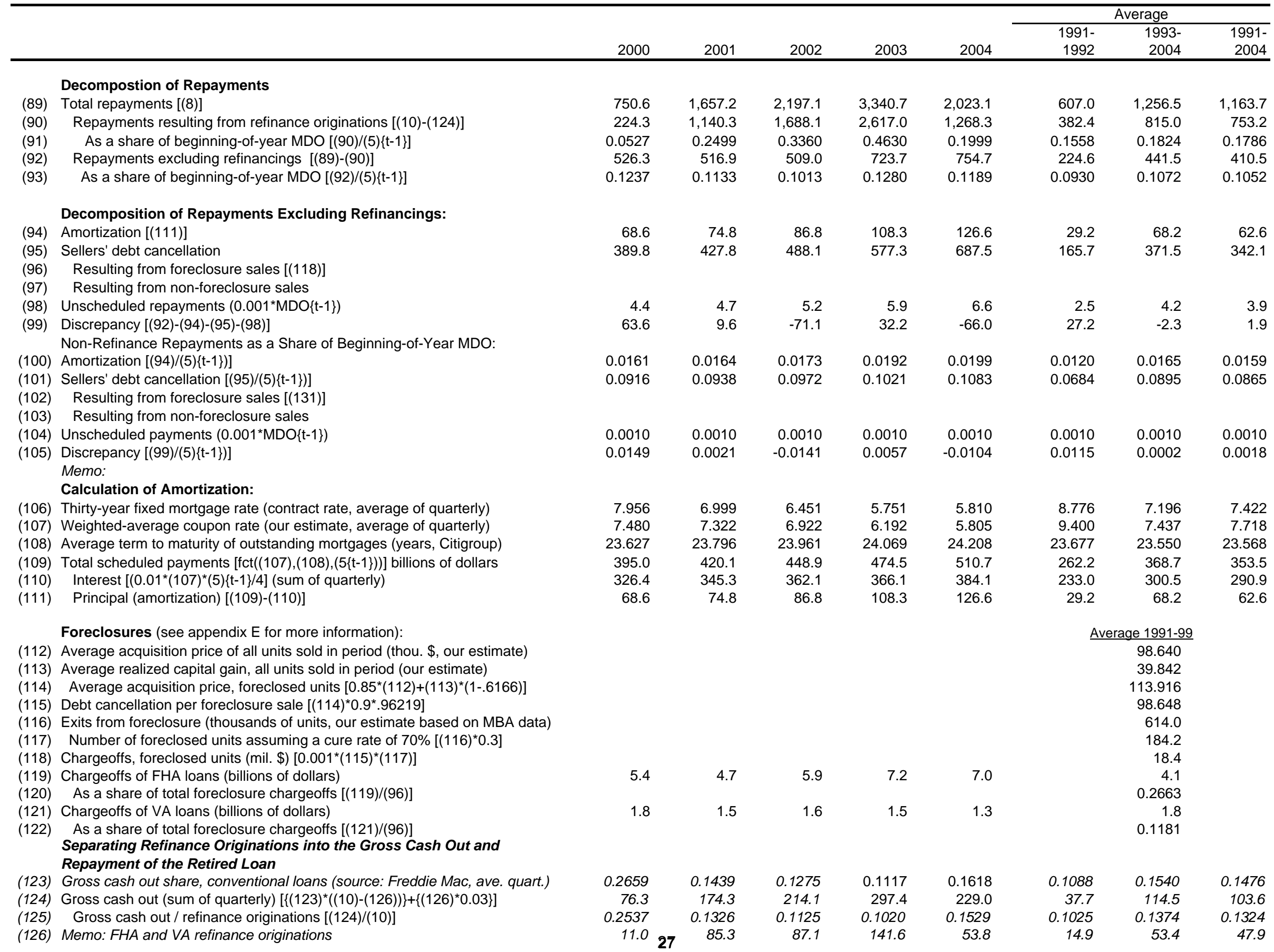


Table 1

Estimates of Mortgage Originations Derived from Mortgage Debt Outstanding and Repayments

(billions of dollars, except where noted; levels are end-of-year values, except where noted)

1991 1992 1993 1994 1995 1996 1997 1998 1999

\section{Commercial Banks}

(127) Originations [(128)*(144)] (sum of quarterly)

(128) Blowup factor: (135)\{t-1\}/(150) (average of quarterly)

(129) Purchases $\left[(128)^{\star}(146)\right]$ (sum of quarterly)

(130) Sales $\left[(128)^{\star}(148)\right]$ (sum of quarterly)

(131) Net sales [(130)-(129)]

(132) 1-4 mortgage debt outstanding, end of year (FOF)

(133) Construction loans

(134) Home equity loans

(135) Regular mortgage debt [(132)-(133)-(134)]

(136) Change

(137) Repayment rate $[(138) /(135)\{t-1\}]$

(138) Repayments (sum of quarterly)

(139) HMDA: Refinance share of originations

(140) Refinance originations [(139)*(127)]

(141) Percent to payoff retired loans [1-(125)]

(142) Repayments net of refinancings $\left[(93)^{\star}(135)\{t-1\}\right]$ (sum of quarterly)

(143) Repayment rate net of refinancings [(142)/(135)\{t-1\})]

Memo:

(144) HMDA originations

(145) HMDA originations / total originations at commercial banks [(144)/(127)]

(146) HMDA purchases

(147) HMDA sales

(148) Adjusted and temporally reallocated

(149) Raw HMDA sales / adjusted HMDA sales [(147)/(148)]

(150) Regular mort. debt outstanding beg. of year (call reports, HMDA filers)

(151) Regular mort. debt outstanding end of year (call reports, HMDA filers)

(152) Change [(151)-(151)\{t-1\}]

(153) Resulting from M\&A and changes in HMDA sample [(150)-(151)\{t-1\}]

(154) Resulting from acquisitions net of sales and repayments [(151)-(150)]

(155) Mergers and acquisitions $\left[(136)-(128)^{*}(154)=(136)-(127)+(131)+(138)\right]$

(156) Sales / (originations + purchases) $[(130) /\{(127)+(129)\}]$

\begin{tabular}{|c|c|c|c|c|c|c|c|c|}
\hline 107.5 & 203.4 & 279.5 & 192.0 & 178.1 & 212.9 & 227.0 & 428.2 & 384.9 \\
\hline 1.1471 & 1.1749 & 1.1639 & 1.1373 & 1.1315 & 1.1277 & 1.1003 & 1.0980 & 1.0915 \\
\hline 58.3 & 88.3 & 91.4 & 65.7 & 60.0 & 104.2 & 108.0 & 179.8 & 187.9 \\
\hline 93.1 & 177.3 & 253.4 & 156.3 & 138.5 & 187.9 & 217.9 & 438.1 & 381.1 \\
\hline 34.9 & 89.0 & 162.0 & 90.6 & 78.6 & 83.7 & 109.9 & 258.3 & 193.2 \\
\hline 452.4 & 478.8 & 532.2 & 590.0 & 646.6 & 677.6 & 745.5 & 797.0 & 879.6 \\
\hline 21.4 & 14.4 & 15.2 & 20.7 & 19.6 & 21.1 & 25.8 & 31.2 & 39.6 \\
\hline 122.8 & 122.7 & 121.3 & 129.7 & 140.0 & 153.8 & 174.0 & 176.9 & 189.5 \\
\hline 308.3 & 341.8 & 395.8 & 439.5 & 487.0 & 502.7 & 545.7 & 589.0 & 650.5 \\
\hline 22.7 & 33.5 & 54.0 & 43.8 & 47.4 & 15.7 & 43.0 & 43.3 & 61.5 \\
\hline 0.2765 & 0.2957 & 0.2292 & 0.1844 & 0.1541 & 0.2747 & 0.2119 & 0.2725 & 0.2667 \\
\hline 78.9 & 91.2 & 78.3 & 73.0 & 67.7 & 133.7 & 106.5 & 148.7 & 157.1 \\
\hline 0.4585 & 0.6563 & 0.6670 & 0.4069 & 0.3298 & 0.4021 & 0.4051 & 0.6177 & 0.4702 \\
\hline 49.3 & 133.5 & 186.4 & 78.1 & 58.7 & 85.6 & 92.0 & 264.5 & 181.0 \\
\hline 0.8673 & 0.9276 & 0.9244 & 0.8711 & 0.8654 & 0.8499 & 0.8540 & 0.8973 & 0.8426 \\
\hline 28.0 & 27.6 & 33.1 & 41.8 & 39.4 & 50.1 & 51.2 & 52.8 & 63.4 \\
\hline 0.0981 & 0.0894 & 0.0968 & 0.1057 & 0.0898 & 0.1029 & 0.1018 & 0.0967 & 0.1076 \\
\hline 93.1 & 173.1 & 239.5 & 169.3 & 157.1 & 189.0 & 205.7 & 389.5 & 353.0 \\
\hline 0.8659 & 0.8509 & 0.8570 & 0.8815 & 0.8818 & 0.8876 & 0.9061 & 0.9096 & 0.9172 \\
\hline 50.5 & 75.1 & 78.4 & 57.9 & 52.9 & 92.3 & 97.9 & 163.4 & 172.3 \\
\hline 75.1 & 140.2 & 199.2 & 116.9 & 113.1 & 152.9 & 181.7 & 371.6 & 307.6 \\
\hline 80.6 & 150.7 & 217.3 & 137.9 & 122.1 & 166.7 & 197.3 & 398.3 & 349.7 \\
\hline 0.9319 & 0.9301 & 0.9166 & 0.8475 & 0.9266 & 0.9168 & 0.9208 & 0.9327 & 0.8798 \\
\hline 262.5 & 266.6 & 298.9 & 352.7 & 393.7 & 439.2 & 468.5 & 504.2 & 543.6 \\
\hline 256.3 & 286.4 & 332.3 & 377.5 & 422.0 & 435.1 & 478.0 & 523.4 & 575.0 \\
\hline 11.3 & 30.1 & 45.9 & 45.2 & 44.4 & 13.1 & 43.0 & 45.4 & 51.5 \\
\hline 17.4 & 10.3 & 12.5 & 20.5 & 16.2 & 17.3 & 33.4 & 26.2 & 20.2 \\
\hline-6.1 & 19.8 & 33.3 & 24.8 & 28.3 & -4.2 & 9.6 & 19.2 & 31.4 \\
\hline 29.1 & 10.2 & 14.8 & 15.3 & 15.6 & 20.3 & 32.4 & 22.1 & 26.9 \\
\hline 0.5619 & 0.6077 & 0.6833 & 0.6065 & 0.5819 & 0.5926 & 0.6504 & 0.7206 & 0.6654 \\
\hline
\end{tabular}


Table 1

Estimates of Mortgage Originations Derived from Mortgage Debt Outstanding and Repayments

(billions of dollars, except where noted; levels are end-of-year values, except where noted)

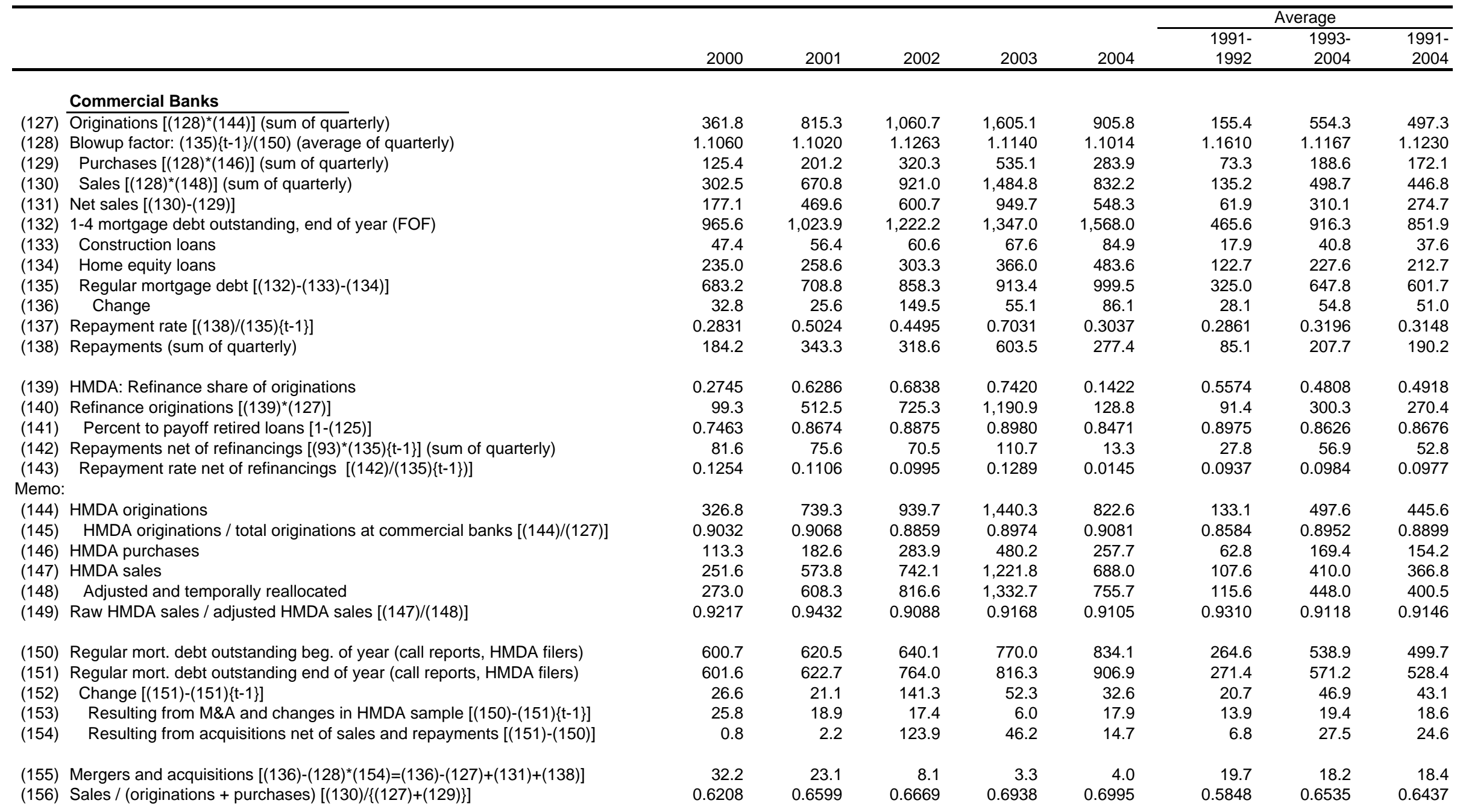


Table 1

Estimates of Mortgage Originations Derived from Mortgage Debt Outstanding and Repayments

(billions of dollars, except where noted; levels are end-of-year values, except where noted)

1991

Thrift Institutions Regulated by OTS

(157) Originations $\left[(158)^{\star}(174)\right]$ (sum of quarterly)

(158) Blowup factor: (165)\{t-1\}/(180) (average of quarterly)

(159) Purchases [(158)*(176)] (sum of quarterly)

(160) Sales $\left[(158)^{\star}(178)\right]$ (sum of quarterly)

(161) Net sales [(160)-(159)]

(162) 1-4 mortgage debt outstanding, end of year (FOF)

(163) Construction loans

(164) Home equity loans

(165) Regular mortgage debt [(162)-(163)-(164)]

(166) Change

(167) Repayment rate $[(168) /(165)\{t-1\}]$

(168) Repayments (sum of quarterly)

(169) HMDA: Refinance share of originations

(170) Refinance originations $\left[(169)^{\star}(157)\right]$

(171) Percent to payoff retired loans [1-(125)]

(172) Repayments net of refinancings $\left[(93)^{\star}(165)\{t-1\}\right]$ (sum of quarterly)

(173) Repayment rate net of refinancings $[(172) /(165)\{t-1\})]$

Memo:

(174) HMDA originations

(175) HMDA originations / total orig. at thrifts regulated by OTS [(174)/(157)

(176) HMDA purchases

(177) HMDA sales

(178) Adjusted and temporally reallocated

(179) Raw HMDA sales / adjusted HMDA sales [(177)/(178)]

(180) Regular mort. debt outstanding beg. of year (call reports, HMDA filers)

(181) Regular mort. debt outstanding end of year (call reports, HMDA filers)

(182) Change [(181)-(181)\{t-1\}]

(183) Resulting from M\&A and changes in HMDA sample [(180)-(181)\{t-1\}]

(184) Resulting from acquisitions net of sales and repayments [(181)-(180)

(185) Mergers and acquisitions $\left[(166)-(158)^{\star}(184)=(166)-(157)+(161)+(168)\right]$

(186) Sales / (originations + purchases) $[(160) /\{(157)+(159)\}]$

1992

1993

1994

1995

1996

1997

1998

1999

$\begin{array}{rrrrrrrrr}160.6 & 230.5 & 220.1 & 146.8 & 123.4 & 153.2 & 180.8 & 315.2 & 254.6 \\ 1.1989 & 1.1400 & 1.1383 & 1.1151 & 1.1149 & 1.0932 & 1.1195 & 1.1085 & 1.1521 \\ 35.4 & 37.4 & 29.3 & 31.2 & 27.4 & 35.5 & 44.6 & 58.7 & 77.9 \\ 98.0 & 147.8 & 140.4 & 76.8 & 62.9 & 84.1 & 108.6 & 208.7 & 184.8 \\ 62.5 & 110.4 & 111.1 & 45.6 & 35.5 & 48.6 & 64.0 & 150.0 & 106.9 \\ 440.7 & 395.5 & 373.8 & 378.0 & 374.5 & 399.3 & 408.5 & 418.2 & 428.6 \\ 11.0 & 9.7 & 10.2 & 10.8 & 11.2 & 12.9 & 8.0 & 9.0 & 10.7 \\ 49.0 & 44.5 & 40.5 & 40.4 & 40.0 & 41.0 & 45.9 & 46.1 & 49.1 \\ 380.7 & 341.3 & 323.0 & 326.8 & 323.3 & 345.4 & 354.6 & 363.0 & 368.8 \\ -56.4 & -39.4 & -18.3 & 3.8 & -3.5 & 22.1 & 9.2 & 8.4 & 5.8 \\ 0.2589 & 0.3474 & 0.3181 & 0.2274 & 0.2101 & 0.2219 & 0.2582 & 0.3922 & 0.3183 \\ 113.2 & 132.3 & 108.6 & 73.5 & 68.7 & 71.8 & 89.2 & 139.1 & 115.5 \\ & & & & & & & & 0.5 \\ 0.4523 & 0.6461 & 0.6617 & 0.3878 & 0.3131 & 0.3730 & 0.3840 & 0.6134 & 0.4600 \\ 72.6 & 148.9 & 145.6 & 56.9 & 38.6 & 57.2 & 69.4 & 193.4 & 117.1 \\ 0.8673 & 0.9276 & 0.9244 & 0.8711 & 0.8654 & 0.8499 & 0.8540 & 0.8973 & 0.8426 \\ 39.5 & 31.4 & 31.4 & 32.8 & 28.2 & 34.2 & 34.5 & 33.4 & 39.8 \\ 0.0902 & 0.0824 & 0.0920 & 0.1017 & 0.0862 & 0.1058 & 0.1000 & 0.0943 & 0.1096 \\ & & & & & & & & \\ 134.6 & 202.3 & 194.1 & 131.4 & 111.0 & 140.2 & 161.8 & 284.7 & 220.9 \\ 0.8379 & 0.8777 & 0.8819 & 0.8952 & 0.8990 & 0.9148 & 0.8952 & 0.9032 & 0.8674 \\ 29.7 & 32.9 & 25.8 & 28.1 & 24.7 & 32.5 & 40.0 & 53.1 & 67.6 \\ 74.7 & 118.4 & 107.9 & 55.2 & 51.7 & 69.4 & 88.2 & 173.7 & 140.8 \\ 82.1 & 129.8 & 123.8 & 68.5 & 56.6 & 76.9 & 97.3 & 188.5 & 160.2 \\ 0.9101 & 0.9118 & 0.8720 & 0.8056 & 0.9127 & 0.9023 & 0.9058 & 0.9213 & 0.8784 \\ 348.9 & & & & & & & & 313 \\ 336.3 & 323.9 & 291.6 & 284.2 & 287.7 & 292.6 & 303.6 & 314.4 & 313.7 \\ -29.3 & -23.1 & 300.8 & 309.5 & 304.9 & 322.6 & 328.2 & 338.0 & 341.7 \\ -16.7 & -12.4 & -21.0 & 8.7 & -4.6 & 17.7 & 5.6 & 9.8 & 3.7 \\ -12.7 & -10.7 & 0.5 & -16.6 & -21.8 & -12.3 & -19.0 & -13.8 & -24.3 \\ & & 25.3 & 17.2 & 30.0 & 24.6 & 23.6 & 28.0 \\ -41.3 & -27.3 & -18.7 & -23.9 & -22.8 & -10.8 & -18.3 & -17.8 & -26.4 \\ 0.4997 & 0.5516 & 0.5629 & 0.4315 & 0.4169 & 0.4456 & 0.4821 & 0.5581 & 0.5558 \\ & & & & & & & & \end{array}$


Table 1

Estimates of Mortgage Originations Derived from Mortgage Debt Outstanding and Repayments

(billions of dollars, except where noted; levels are end-of-year values, except where noted)

\begin{tabular}{|c|c|c|c|c|c|c|c|c|c|}
\hline & & \multirow[b]{2}{*}{2000} & \multirow[b]{2}{*}{2001} & \multirow[b]{2}{*}{2002} & \multirow[b]{2}{*}{2003} & \multirow[b]{2}{*}{2004} & \multicolumn{3}{|c|}{ Average } \\
\hline & & & & & & & $\begin{array}{r}1991- \\
1992 \\
\end{array}$ & $\begin{array}{r}1993- \\
2004 \\
\end{array}$ & $\begin{array}{r}1991- \\
2004 \\
\end{array}$ \\
\hline & \multicolumn{9}{|l|}{ Thrift Institutions Regulated by OTS } \\
\hline (157) & Originations [(158)*(174)] (sum of quarterly) & 210.1 & 399.1 & 514.7 & 647.0 & 457.2 & 195.6 & 301.9 & 286.7 \\
\hline (158) & Blowup factor: (165)\{t-1\}/(180) (average of quarterly) & 1.0892 & 1.1150 & 1.0879 & 1.1095 & 0.9927 & 1.1695 & 1.1030 & 1.1125 \\
\hline (159) & Purchases $\left[(158)^{*}(176)\right]$ (sum of quarterly) & 78.1 & 187.5 & 213.1 & 294.4 & 252.9 & 36.4 & 110.9 & 100.2 \\
\hline (160) & Sales $\left[(158)^{\star}(178)\right]$ (sum of quarterly) & 149.6 & 366.2 & 528.6 & 742.1 & 451.0 & 122.9 & 258.6 & 239.3 \\
\hline (161) & Net sales [(160)-(159)] & 71.5 & 178.7 & 315.5 & 447.8 & 198.1 & 86.4 & 147.8 & 139.0 \\
\hline (162) & 1-4 mortgage debt outstanding, end of year (FOF) & 472.4 & 490.9 & 504.9 & 566.5 & 754.5 & 418.1 & 464.2 & 457.6 \\
\hline (163) & Construction loans & 12.5 & 12.6 & 12.0 & 12.8 & 15.9 & 10.4 & 11.6 & 11.4 \\
\hline (164) & Home equity loans & 61.5 & 65.0 & 63.3 & 75.9 & 103.6 & 46.7 & 56.0 & 54.7 \\
\hline (165) & Regular mortgage debt [(162)-(163)-(164)] & 398.4 & 413.2 & 429.6 & 477.7 & 635.1 & 361.0 & 396.6 & 391.5 \\
\hline$(166)$ & Change & 29.6 & 14.8 & 16.4 & 48.2 & 157.3 & -47.9 & 24.5 & 14.1 \\
\hline (167) & Repayment rate $[(168) /(165)\{t-1\}]$ & 0.2807 & 0.4612 & 0.4407 & 0.3544 & 0.3096 & 0.3031 & 0.3161 & 0.3142 \\
\hline$(168)$ & Repayments (sum of quarterly) & 103.5 & 183.8 & 182.1 & 152.3 & 147.9 & 122.7 & 119.7 & 120.1 \\
\hline$(169)$ & HMDA: Refinance share of originations & 0.3047 & 0.6594 & 0.7163 & 0.7594 & 0.5973 & 0.5492 & 0.5192 & 0.5234 \\
\hline (170) & Refinance originations [(169)*(157)] & 64.0 & 263.2 & 368.7 & 491.3 & 273.1 & 110.8 & 178.2 & 168.6 \\
\hline (171) & Percent to payoff retired loans [1-(125)] & 0.7463 & 0.8674 & 0.8875 & 0.8980 & 0.8471 & 0.8975 & 0.8626 & 0.8676 \\
\hline (172) & Repayments net of refinancings $[(93) \star(165)\{t-1\}]$ (sum of quarterly) & 46.2 & 44.7 & 39.8 & 55.7 & 62.5 & 35.4 & 40.3 & 39.6 \\
\hline (173) & Repayment rate net of refinancings [(172)/(165)\{t-1\})] & 0.1254 & 0.1121 & 0.0963 & 0.1296 & 0.1308 & 0.0863 & 0.1070 & 0.1040 \\
\hline \multicolumn{10}{|c|}{ 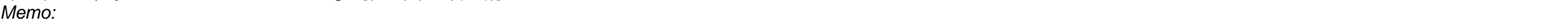 } \\
\hline$(174)$ & HMDA originations & 193.1 & 358.4 & 474.0 & 583.2 & 459.5 & 168.4 & 276.0 & 260.6 \\
\hline (175) & HMDA originations / total orig. at thrifts regulated by OTS [(174)/(157)] & 0.9189 & 0.8980 & 0.9209 & 0.9014 & 1.0050 & 0.8578 & 0.9084 & 0.9012 \\
\hline$(176)$ & HMDA purchases & 71.7 & 168.5 & 196.2 & 265.3 & 254.1 & 31.3 & 102.3 & 92.2 \\
\hline (177) & HMDA sales & 125.7 & 306.9 & 437.0 & 599.9 & 416.0 & 96.6 & 214.4 & 197.5 \\
\hline (178) & Adjusted and temporally reallocated & 137.5 & 329.1 & 486.6 & 668.9 & 453.2 & 106.0 & 237.3 & 218.5 \\
\hline$(179)$ & Raw HMDA sales / adjusted HMDA sales [(177)/(178)] & 0.9144 & 0.9328 & 0.8979 & 0.8968 & 0.9179 & 0.9110 & 0.8965 & 0.8986 \\
\hline (180) & Regular mort. debt outstanding beg. of year (call reports, HMDA filers) & 334.5 & 353.2 & 375.3 & 387.9 & 506.0 & 336.4 & 337.1 & 337.0 \\
\hline (181) & Regular mort. debt outstanding end of year (call reports, HMDA filers) & 366.6 & 385.9 & 391.3 & 430.2 & 617.8 & 324.7 & 369.8 & 363.4 \\
\hline$(182)$ & Change $[(181)-(181)\{t-1\}]$ & 25.0 & 19.3 & 5.4 & 38.9 & 187.5 & -26.2 & 24.7 & 17.4 \\
\hline (183) & Resulting from M\&A and changes in HMDA sample [(180)-(181)\{t-1\}] & -7.2 & -13.5 & -10.6 & -3.5 & 75.8 & -14.6 & -7.4 & -8.4 \\
\hline$(184)$ & Resulting from acquisitions net of sales and repayments [(181)-(180)] & 32.2 & 32.8 & 16.0 & 42.4 & 111.8 & -11.7 & 32.0 & 25.8 \\
\hline$(185)$ & Mergers and acquisitions $\left[(166)-(158)^{\star}(184)=(166)-(157)+(161)+(168)\right]$ & -5.5 & -21.8 & -0.7 & 1.2 & 46.1 & -34.3 & -10.0 & -13.4 \\
\hline (186) & Sales / (originations + purchases) $[(160) /\{(157)+(159)\}]$ & 0.5189 & 0.6243 & 0.7263 & 0.7883 & 0.6351 & 0.5256 & 0.5621 & 0.5569 \\
\hline
\end{tabular}


Table 1

Estimates of Mortgage Originations Derived from Mortgage Debt Outstanding and Repayments

(billions of dollars, except where noted; levels are end-of-year values, except where noted)

1991

(187) Originations $\left[(188)^{\star}(204)\right]$ (sum of quarterly)

(188) Blowup factor: (195)\{t-1\}/(210) (average of quarterly)

(189) Purchases $\left[(188)^{\star}(206)\right]$ (sum of quarterly)

(190) Sales $\left[(188)^{\star}(208)\right]$ (sum of quarterly)

(191) Net sales [(190)-(189)]

(192) 1-4 mortgage debt outstanding, end of year (FOF)

(193) Construction loans

(194) Home equity loans

(195) Regular mortgages [(192)-(193)-(194)]

(196) Change

(197) Repayment rate $[(198) /(195)\{t-1\}]$

(198) Repayments (sum of quarterly)

(199) HMDA: Refinance share of originations

(200) Refinance originations [(199)*(187)]

(201) Percent to payoff retired loans [1-(125)]

(202) Repayments net of refinancings [(93)*(195)\{t-1\}] (sum of quarterly)

(203) Repayment rate net of refinancings [(202)/(195)\{t-1\})]

Memo:

(204) HMDA originations

(205) HMDA originations / total originations at savings banks [(204)/(187)]

(206) HMDA purchases

(207) HMDA sales

(208) Adjusted and temporally reallocated

(209) Raw HMDA sales / adjusted HMDA sales [(207)/(208)]

(210) Regular mort. debt outstanding beg. of year (call reports, HMDA filers)

(211) Regular mort. debt outstanding end of year (call reports, HMDA filers)

(212) Change [(211)-(211) \{t-1\}]

(213) Resulting from M\&A and changes in HMDA sample [(210)-(211)\{t-1\}]

(214) Resulting from acquisitions net of sales and repayments [(211)-(210)

(215) Mergers and acquisitions $\left[(196)-(188)^{\star}(214)=(196)-(187)+(191)+(198)\right]$

(216) Sales / (originations + purchases) $[(190) /\{(187)+(189)\}]$

1992

1993

1994

1995

1996

1997

1998

1999

\begin{tabular}{|c|c|c|c|c|c|c|c|c|}
\hline 20.8 & 42.7 & 50.6 & 33.2 & 26.2 & 37.9 & 38.2 & 62.2 & 51.7 \\
\hline 2.9917 & 1.9721 & 1.1755 & 1.0275 & 0.9964 & 1.0536 & 1.1111 & 1.0773 & 1.0554 \\
\hline 1.4 & 4.2 & 8.6 & 7.8 & 4.2 & 5.2 & 7.5 & 11.2 & 8.4 \\
\hline 6.3 & 17.6 & 28.5 & 18.7 & 10.9 & 17.1 & 19.4 & 39.0 & 32.8 \\
\hline 4.8 & 13.4 & 19.9 & 10.9 & 6.8 & 11.9 & 12.0 & 27.8 & 24.4 \\
\hline 97.7 & 94.1 & 96.2 & 99.6 & 107.9 & 114.4 & 112.2 & 115.3 & 119.6 \\
\hline 1.0 & 0.7 & 1.1 & 1.7 & 1.9 & 2.7 & 2.9 & 3.4 & 4.0 \\
\hline 9.8 & 9.1 & 9.3 & 9.1 & 10.5 & 11.2 & 9.5 & 9.8 & 10.6 \\
\hline 86.9 & 84.2 & 85.8 & 88.8 & 95.4 & 100.5 & 99.7 & 102.1 & 105.1 \\
\hline 1.9 & -2.7 & 1.6 & 3.0 & 6.6 & 5.1 & -0.8 & 2.4 & 2.9 \\
\hline 0.1869 & 0.2754 & 0.2809 & 0.2156 & 0.1782 & 0.1806 & 0.1819 & 0.2774 & 0.2253 \\
\hline 15.9 & 23.9 & 23.7 & 18.5 & 15.8 & 17.2 & 18.3 & 27.7 & 23.0 \\
\hline 0.4381 & 0.6207 & 0.6618 & 0.3854 & 0.3214 & 0.3680 & 0.3721 & 0.5942 & 0.4400 \\
\hline 9.1 & 26.5 & 33.5 & 12.8 & 8.4 & 14.0 & 14.2 & 36.9 & 22.7 \\
\hline 0.8673 & 0.9276 & 0.9244 & 0.8711 & 0.8654 & 0.8499 & 0.8540 & 0.8973 & 0.8426 \\
\hline 8.4 & 7.3 & 7.7 & 8.9 & 7.7 & 9.9 & 9.9 & 9.5 & 11.2 \\
\hline 0.0986 & 0.0838 & 0.0910 & 0.1033 & 0.0862 & 0.1040 & 0.0985 & 0.0951 & 0.1094 \\
\hline 7.0 & 21.9 & 45.3 & 32.3 & 26.3 & 36.0 & 34.5 & 57.7 & 48.9 \\
\hline 0.3354 & 0.5118 & 0.8948 & 0.9723 & 1.0039 & 0.9491 & 0.9031 & 0.9286 & 0.9468 \\
\hline 0.5 & 2.0 & 7.8 & 7.5 & 4.2 & 4.9 & 6.7 & 10.4 & 7.9 \\
\hline 2.0 & 8.8 & 25.1 & 15.7 & 10.3 & 15.2 & 16.5 & 34.4 & 28.0 \\
\hline 2.1 & 9.0 & 25.9 & 18.1 & 11.0 & 16.2 & 17.6 & 36.2 & 31.0 \\
\hline 0.9688 & 0.9717 & 0.9692 & 0.8637 & 0.9378 & 0.9404 & 0.9383 & 0.9509 & 0.9011 \\
\hline 27.8 & 37.3 & 50.8 & 83.4 & 89.0 & 89.1 & 87.9 & 91.1 & 96.4 \\
\hline 30.1 & 46.7 & 82.8 & 87.0 & 92.6 & 97.5 & 95.1 & 97.3 & 100.4 \\
\hline-0.8 & 9.9 & 9.8 & 4.2 & 5.5 & 4.9 & -2.4 & 2.2 & 3.0 \\
\hline-0.8 & 7.2 & 4.1 & 0.5 & 2.0 & -3.4 & -9.6 & -4.0 & -1.0 \\
\hline 0.0 & 2.7 & 5.7 & 3.7 & 3.6 & 8.4 & 7.2 & 6.2 & 4.0 \\
\hline 1.9 & -8.1 & -5.5 & -0.7 & 3.0 & -3.8 & -8.8 & -4.3 & -1.3 \\
\hline 0.2825 & 0.3751 & 0.4805 & 0.4562 & 0.3605 & 0.3957 & 0.4249 & 0.5316 & 0.5463 \\
\hline
\end{tabular}


Table 1

Estimates of Mortgage Originations Derived from Mortgage Debt Outstanding and Repayments

(billions of dollars, except where noted; levels are end-of-year values, except where noted)

\begin{tabular}{|c|c|c|c|c|c|c|c|c|c|}
\hline & & & & & & & \multicolumn{3}{|c|}{ Average } \\
\hline & & 2000 & 2001 & 2002 & 2003 & 2004 & $\begin{array}{r}1991- \\
1992 \\
\end{array}$ & $\begin{array}{r}1993- \\
2004 \\
\end{array}$ & $\begin{array}{r}1991- \\
2004 \\
\end{array}$ \\
\hline & \multicolumn{9}{|l|}{ Savings Banks (thrift institutions regulated by the FDIC) } \\
\hline (187) & Originations [(188)*(204)] (sum of quarterly) & 39.9 & 79.6 & 98.1 & 123.4 & 95.6 & 31.7 & 61.4 & 57.2 \\
\hline (188) & Blowup factor: (195)\{t-1\}/(210) (average of quarterly) & 1.0703 & 1.0038 & 1.0529 & 1.0180 & 1.1629 & 2.4819 & 1.0671 & 1.2692 \\
\hline (189) & Purchases $\left[(188)^{\star}(206)\right]$ (sum of quarterly) & 6.3 & 12.4 & 17.1 & 25.8 & 10.3 & 2.8 & 10.4 & 9.3 \\
\hline (190) & Sales $\left[(188)^{\star}(208)\right]$ (sum of quarterly) & 22.9 & 56.3 & 79.6 & 103.6 & 76.3 & 11.9 & 42.1 & 37.8 \\
\hline (191) & Net sales [(190)-(189)] & 16.6 & 44.0 & 62.6 & 77.8 & 66.0 & 9.1 & 31.7 & 28.5 \\
\hline (192) & 1-4 mortgage debt outstanding, end of year (FOF) & 121.8 & 129.7 & 126.5 & 136.9 & 121.3 & 95.9 & 116.8 & 113.8 \\
\hline (193) & Construction loans & 4.2 & 5.6 & 5.5 & 6.4 & 6.6 & 0.9 & 3.8 & 3.4 \\
\hline (194) & Home equity loans & 11.3 & 12.9 & 15.2 & 19.7 & 17.9 & 9.5 & 12.2 & 11.9 \\
\hline (195) & Regular mortgages [(192)-(193)-(194)] & 106.3 & 111.2 & 105.8 & 110.8 & 96.8 & 85.6 & 100.7 & 98.5 \\
\hline (196) & Change & 1.2 & 5.0 & -5.4 & 5.0 & -14.0 & -0.4 & 1.0 & 0.8 \\
\hline (197) & Repayment rate $[(198) /(195)\{t-1\}]$ & 0.1338 & 0.2730 & 0.3384 & 0.3571 & 0.1721 & 0.2311 & 0.2345 & 0.2340 \\
\hline (198) & Repayments (sum of quarterly) & 14.1 & 29.0 & 37.6 & 37.8 & 19.1 & 19.9 & 23.5 & 23.0 \\
\hline (199) & HMDA: Refinance share of originations & 0.2657 & 0.6120 & 0.6749 & 0.7209 & 0.5161 & 0.5294 & 0.4944 & 0.4994 \\
\hline (200) & Refinance originations $\left[(199)^{\star}(187)\right]$ & 10.6 & 48.7 & 66.2 & 88.9 & 49.3 & 17.8 & 33.9 & 31.6 \\
\hline (201) & Percent to payoff retired loans [1-(125)] & 0.7463 & 0.8674 & 0.8875 & 0.8980 & 0.8471 & 0.8975 & 0.8626 & 0.8676 \\
\hline (202) & Repayments net of refinancings $\left[(93)^{\star}(195)\{t-1\}\right]$ (sum of quarterly) & 12.9 & 12.0 & 10.6 & 13.3 & 12.1 & 7.8 & 10.5 & 10.1 \\
\hline$(203)$ & Repayment rate net of refinancings $[(202) /(195)\{t-1\})]$ & 0.1232 & 0.1131 & 0.0955 & 0.1256 & 0.1092 & 0.0912 & 0.1045 & 0.1026 \\
\hline \multicolumn{10}{|c|}{ Memo: } \\
\hline (204) & HMDA originations & 37.3 & 79.2 & 93.3 & 121.2 & 82.3 & 14.4 & 57.9 & 51.6 \\
\hline (205) & HMDA originations / total originations at savings banks [(204)/(187)] & 0.9350 & 0.9947 & 0.9506 & 0.9819 & 0.8605 & 0.4236 & 0.9434 & 0.8692 \\
\hline$(206)$ & HMDA purchases & 5.9 & 12.3 & 16.2 & 25.4 & 8.9 & 1.2 & 9.9 & 8.6 \\
\hline (207) & HMDA sales & 19.8 & 54.1 & 70.4 & 92.2 & 60.7 & 5.4 & 36.9 & 32.4 \\
\hline$(208)$ & Adjusted and temporally reallocated & 21.4 & 56.0 & 75.7 & 101.7 & 65.5 & 5.6 & 39.7 & 34.8 \\
\hline (209) & Raw HMDA sales / adjusted HMDA sales [(207)/(208)] & 0.9264 & 0.9663 & 0.9297 & 0.9058 & 0.9261 & 0.9702 & 0.9296 & 0.9354 \\
\hline (210) & Regular mort. debt outstanding beg. of year (call reports, HMDA filers) & 96.5 & 107.6 & 104.7 & 102.9 & 89.6 & 32.6 & 90.7 & 82.4 \\
\hline (211) & Regular mort. debt outstanding end of year (call reports, HMDA filers) & 105.2 & 114.1 & 102.7 & 110.6 & 98.8 & 38.4 & 98.7 & 90.1 \\
\hline (212) & Change $[(211)-(211)\{t-1\}]$ & 4.8 & 9.0 & -11.4 & 7.8 & -11.8 & 4.6 & 2.1 & 2.5 \\
\hline (213) & Resulting from M\&A and changes in HMDA sample [(210)-(211)\{t-1\}] & -3.8 & 2.4 & -9.5 & 0.1 & -21.0 & 3.2 & -3.6 & -2.6 \\
\hline$(214)$ & Resulting from acquisitions net of sales and repayments [(211)-(210)] & 8.7 & 6.6 & -2.0 & 7.7 & 9.2 & 1.3 & 5.7 & 5.1 \\
\hline$(215)$ & Mergers and acquisitions $[(196)-(188) \star(214)=(196)-(187)+(191)+(198)]$ & -8.1 & -1.7 & -3.3 & -2.8 & -24.6 & -3.1 & -5.2 & -4.9 \\
\hline$(216)$ & Sales / (originations + purchases) $[(190) /\{(187)+(189)\}]$ & 0.4947 & 0.6123 & 0.6914 & 0.6943 & 0.7202 & 0.3288 & 0.5340 & 0.5047 \\
\hline
\end{tabular}


Table 1

Estimates of Mortgage Originations Derived from Mortgage Debt Outstanding and Repayments

(billions of dollars, except where noted; levels are end-of-year values, except where noted)

\begin{tabular}{|c|c|c|c|c|c|c|c|c|c|c|}
\hline & & 1991 & 1992 & 1993 & 1994 & 1995 & 1996 & 1997 & 1998 & 1999 \\
\hline & \multicolumn{10}{|l|}{ Credit Unions } \\
\hline (217) & Originations $[(221)+(226)+(228)]$ & 9.5 & 17.1 & 21.3 & 14.1 & 10.9 & 17.0 & 18.6 & 34.1 & 29.9 \\
\hline$(218)$ & Blowup factor: (225)\{t-1\}/(238)\{t-1\} (average of quarterly) & 1.0882 & 1.0775 & 1.0926 & 1.1015 & 1.0852 & 1.0829 & 1.0828 & 1.0742 & 1.0702 \\
\hline (219) & Purchases $\left[(218)^{\star}(241)\right]$ (sum of quarterly) & 0.2 & 0.3 & 0.4 & 0.2 & 0.1 & 0.2 & 0.2 & 0.3 & 0.4 \\
\hline (220) & Sales $\left[(218)^{\star}(242)\right]$ (sum of quarterly) & 4.0 & 7.4 & 9.9 & 4.1 & 3.4 & 4.0 & 4.9 & 11.7 & 7.7 \\
\hline (221) & Net sales [(220)-(219)] & 3.9 & 7.1 & 9.5 & 4.0 & 3.3 & 3.8 & 4.6 & 11.3 & 7.3 \\
\hline (222) & 1-4 mortgage debt outstanding, end of year (FOF) & 52.8 & 53.8 & 56.0 & 62.1 & 66.5 & 76.0 & 86.0 & 96.9 & 111.0 \\
\hline (223) & Construction loans & 0.0 & 0.0 & 0.0 & 0.0 & 0.0 & 0.0 & 0.0 & 0.0 & 0.0 \\
\hline (224) & Home equity loans & 21.4 & 20.5 & 19.3 & 20.9 & 22.9 & 25.5 & 29.0 & 29.7 & 33.4 \\
\hline (225) & Regular mortgages [(222)-(223)-(224)] & 31.4 & 33.3 & 36.7 & 41.3 & 43.6 & 50.5 & 57.0 & 67.1 & 77.6 \\
\hline (226) & Change & 1.9 & 1.9 & 3.4 & 4.6 & 2.3 & 6.9 & 6.6 & 10.1 & 10.5 \\
\hline$(227)$ & Repayment rate $[(228) /(225)\{t-1\}]$ & 0.1275 & 0.2570 & 0.2505 & 0.1530 & 0.1294 & 0.1439 & 0.1459 & 0.2212 & 0.1809 \\
\hline (228) & Repayments (sum of quarterly) & 3.8 & 8.1 & 8.3 & 5.6 & 5.3 & 6.3 & 7.4 & 12.6 & 12.1 \\
\hline (229) & HMDA: Refinance share of originations & 0.6037 & 0.8075 & 0.7753 & 0.5405 & 0.4631 & 0.5220 & 0.4822 & 0.7101 & 0.5500 \\
\hline (230) & Refinance originations $[(229) \star(217)]$ & 5.7 & 13.8 & 16.5 & 7.6 & 5.1 & 8.9 & 9.0 & 24.2 & 16.5 \\
\hline (231) & Percent to payoff retired loans [1-(125)] & 0.8673 & 0.9276 & 0.9244 & 0.8711 & 0.8654 & 0.8499 & 0.8540 & 0.8973 & 0.8426 \\
\hline (232) & Repayments net of refinancings $\left[(93)^{\star}(225)\{t-1\}\right]$ (sum of quarterly) & 2.8 & 2.7 & 3.2 & 4.0 & 3.6 & 4.8 & 5.2 & 5.7 & 7.7 \\
\hline$(233)$ & Repayment rate net of refinancings $[(232) /(225)\{t-1\})]$ & 0.0962 & 0.0848 & 0.0964 & 0.1088 & 0.0866 & 0.1093 & 0.1032 & 0.0998 & 0.1142 \\
\hline \multicolumn{11}{|c|}{ Memo: } \\
\hline (234) & HMDA originations & 5.4 & 15.5 & 19.4 & 13.3 & 8.3 & 13.0 & 13.7 & 25.7 & 22.5 \\
\hline$(235)$ & HMDA originations / total originations at credit unions $[(234) /(217)]$ & 0.5711 & 0.9073 & 0.9142 & 0.9418 & 0.7605 & 0.7627 & 0.7378 & 0.7538 & 0.7506 \\
\hline$(236)$ & HMDA purchases & 0.0 & 0.0 & 0.2 & 0.2 & 0.1 & 0.1 & 0.1 & 0.1 & 0.1 \\
\hline$(237)$ & HMDA sales (unadjusted) & 2.0 & 5.4 & 6.4 & 2.3 & 2.0 & 2.8 & 3.4 & 6.6 & 4.8 \\
\hline$(238)$ & Regular Mortgage debt outstanding (NCUA filers) & 28.0 & 30.4 & 33.5 & 38.1 & 40.1 & 46.4 & 52.7 & 62.3 & 72.1 \\
\hline$(239)$ & NCUA originations & 7.5 & 16.6 & 19.4 & 13.2 & 10.0 & 15.6 & 17.3 & 31.9 & 28.0 \\
\hline$(240)$ & NCUA originations / total originations at credit unions $[(239) /(217)]$ & 0.7885 & 0.9696 & 0.9146 & 0.9374 & 0.9105 & 0.9199 & 0.9328 & 0.9372 & 0.9366 \\
\hline$(241)$ & NCUA purchases & 0.1 & 0.3 & 0.4 & 0.1 & 0.1 & 0.2 & 0.2 & 0.3 & 0.3 \\
\hline (242) & NCUA sales & 3.7 & 6.9 & 9.1 & 3.7 & 3.1 & 3.7 & 4.5 & 10.9 & 7.2 \\
\hline (243) & HMDA originations / NCUA originations & 0.7243 & 0.9358 & 0.9995 & 1.0047 & 0.8353 & 0.8291 & 0.7910 & 0.8044 & 0.8014 \\
\hline$(244)$ & Sales /(originations + purchases) $[(220) /\{(217)+(219)\}]$ & 0.4155 & 0.4284 & 0.4571 & 0.2890 & 0.3069 & 0.2351 & 0.2595 & 0.3395 & 0.2531 \\
\hline
\end{tabular}


Table 1

Estimates of Mortgage Originations Derived from Mortgage Debt Outstanding and Repayments

(billions of dollars, except where noted; levels are end-of-year values, except where noted)

\begin{tabular}{|c|c|c|c|c|c|c|c|c|c|}
\hline & & \multirow[b]{2}{*}{2000} & \multirow[b]{2}{*}{2001} & \multirow[b]{2}{*}{2002} & \multirow[b]{2}{*}{2003} & \multirow[b]{2}{*}{2004} & \multicolumn{3}{|c|}{ Average } \\
\hline & & & & & & & $\begin{array}{r}1991- \\
1992\end{array}$ & $\begin{array}{r}1993- \\
2004\end{array}$ & $\begin{array}{r}1991- \\
2004\end{array}$ \\
\hline & \multicolumn{9}{|l|}{ Credit Unions } \\
\hline$(217)$ & $\overline{\text { Originations }[(221)+(226)+(228)]}$ & 22.6 & 48.4 & 69.3 & 95.9 & 67.0 & 13.3 & 37.4 & 34.0 \\
\hline (218) & Blowup factor: (225)\{t-1\}/(238)\{t-1\} (average of quarterly) & 1.0707 & 1.0760 & 1.0784 & 1.0866 & 1.1011 & 1.0828 & 1.0835 & 1.0834 \\
\hline (219) & Purchases [(218)*(241)] (sum of quarterly) & 0.6 & 0.6 & 0.7 & 2.6 & 1.9 & 0.2 & 0.7 & 0.6 \\
\hline (220) & Sales [(218)*(242)] (sum of quarterly) & 6.4 & 17.8 & 27.1 & 40.8 & 22.1 & 5.7 & 13.3 & 12.2 \\
\hline (221) & Net sales [(220)-(219)] & 5.8 & 17.3 & 26.4 & 38.3 & 20.2 & 5.5 & 12.7 & 11.6 \\
\hline (222) & 1-4 mortgage debt outstanding, end of year (FOF) & 124.9 & 141.3 & 159.4 & 182.6 & 213.2 & 53.3 & 114.7 & 105.9 \\
\hline (223) & Construction loans & 0.0 & 0.0 & 0.0 & 0.0 & 0.0 & 0.0 & 0.0 & 0.0 \\
\hline (224) & Home equity loans & 40.7 & 44.9 & 48.1 & 51.8 & 64.0 & 21.0 & 35.8 & 33.7 \\
\hline (225) & Regular mortgages [(222)-(223)-(224)] & 84.1 & 96.3 & 111.4 & 130.8 & 149.3 & 32.4 & 78.8 & 72.2 \\
\hline (226) & Change & 6.5 & 12.2 & 15.1 & 19.4 & 18.5 & 1.9 & 9.7 & 8.6 \\
\hline (227) & Repayment rate $[(228) /(225)\{t-1\}]$ & 0.1319 & 0.2250 & 0.2888 & 0.3429 & 0.2166 & 0.1923 & 0.2025 & 0.2010 \\
\hline (228) & Repayments (sum of quarterly) & 10.2 & 18.9 & 27.8 & 38.2 & 28.3 & 5.9 & 15.1 & 13.8 \\
\hline (229) & HMDA: Refinance share of originations & 0.3420 & 0.7058 & 0.7613 & 0.8190 & 0.6132 & 0.7056 & 0.6070 & 0.6211 \\
\hline (230) & Refinance originations $\left[(229)^{\star}(217)\right]$ & 7.7 & 34.2 & 52.7 & 78.5 & 41.1 & 9.8 & 25.2 & 23.0 \\
\hline (231) & Percent to payoff retired loans [1-(125)] & 0.7463 & 0.8674 & 0.8875 & 0.8980 & 0.8471 & 0.8975 & 0.8626 & 0.8676 \\
\hline (232) & Repayments net of refinancings $\left[(93)^{\star}(225)\{t-1\}\right]$ (sum of quarterly) & 9.7 & 9.6 & 10.0 & 14.5 & 15.7 & 2.8 & 7.8 & 7.1 \\
\hline$(233)$ & Repayment rate net of refinancings $[(232) /(225)\{t-1\})]$ & 0.1244 & 0.1146 & 0.1037 & 0.1302 & 0.1199 & 0.0905 & 0.1093 & 0.1066 \\
\hline \multicolumn{10}{|c|}{ Memo: } \\
\hline (234) & HMDA originations & 16.5 & 40.8 & 55.5 & 78.3 & 47.3 & 10.5 & 29.5 & 26.8 \\
\hline (235) & HMDA originations / total originations at credit unions [(234)/(217)] & 0.7309 & 0.8420 & 0.8017 & 0.8166 & 0.7059 & 0.7392 & 0.7932 & 0.7855 \\
\hline (236) & HMDA purchases & 0.1 & 0.2 & 0.3 & 0.8 & 0.9 & 0.0 & 0.3 & 0.2 \\
\hline$(237)$ & HMDA sales (unadjusted) & 3.9 & 9.5 & 18.1 & 26.8 & 16.2 & 3.7 & 8.6 & 7.9 \\
\hline (238) & Regular Mortgage debt outstanding (NCUA filers) & 77.7 & 90.7 & 102.4 & 120.1 & 133.2 & 29.2 & 72.4 & 66.3 \\
\hline (239) & NCUA originations & 20.6 & 46.6 & 61.9 & 88.1 & 57.2 & 12.0 & 34.2 & 31.0 \\
\hline (240) & NCUA originations / total originations at credit unions [(239)/(217)] & 0.9116 & 0.9627 & 0.8933 & 0.9189 & 0.8540 & 0.8790 & 0.9191 & 0.9134 \\
\hline (241) & NCUA purchases & 0.5 & 0.5 & 0.6 & 2.4 & 1.7 & 0.2 & 0.6 & 0.6 \\
\hline$(242)$ & NCUA sales & 5.9 & 16.6 & 25.1 & 37.6 & 20.1 & 5.3 & 12.3 & 11.3 \\
\hline (243) & HMDA originations / NCUA originations & 0.8018 & 0.8746 & 0.8974 & 0.8886 & 0.8265 & 0.8300 & 0.8629 & 0.8582 \\
\hline (244) & Sales /(originations + purchases) $[(220) /\{(217)+(219)\}]$ & 0.2750 & 0.3644 & 0.3871 & 0.4149 & 0.3205 & 0.4219 & 0.3252 & 0.3390 \\
\hline
\end{tabular}


Table 1

Estimates of Mortgage Originations Derived from Mortgage Debt Outstanding and Repayments

(billions of dollars, except where noted; levels are end-of-year values, except where noted)

1991

1992

1993

1994

1995

1996

1997

1998

1999

\section{The GSEs and Ginnie Mae}

Fannie Mae

(246) Sales

(247) Net sales [(246)-(245)]

(248) 1-4 MDO, end of quarter (held in porfolio or in securitized pools)

(249) Construction loans

(250) Home equity loans

(251) Regular mortgages [(248)-(249)-(250)]

(252) Change

(253) Repayment rate $[(254) /(251)\{t-1\}]$

(254) Repayments [(245)-(246)-(252)]

\section{Freddie Mac}

(255) Purchases

(256) Sales

(257) Net sales [(256)-(255)]

(258) 1-4 MDO, end of quarter (held in porfolio or in securitized pools)

(259) Construction loans

(260) Home equity loans

(261) Regular mortgages [(258)-(259)-(260)]

(262) Change

(263) Repayment rate $[(264) /(261)\{t-1\}]$

(264) Repayments [(255)-(256)-(262)]

(265)a Memo: Purchases by Fannie and Freddie as a share of originations Ginnie Mae

(265) Purchases (issuance)

(266) Sales

(267) Net sales [(266)-(265)]

(268) 1-4 MDO, end of quarter (securitized pools)

(269) Construction loans

(270) Home equity loans

(271) Regular mortgages [(268)-(269)-(270)]

(272) Change

(273) Repayment rate $[(274) /(271)\{t-1\}]$

(274) Repayments [(265)-(266)-(272)]

(275) GNM purchases / (VA and FHA originations)

$\begin{array}{rrrrrrrrr}133.6 & 248.6 & 289.8 & 158.2 & 126.0 & 164.5 & 159.9 & 354.9 & 316.1 \\ 0.7 & 0.9 & 0.7 & 0.2 & 0.0 & 0.0 & 0.1 & 0.2 & 0.6 \\ -132.8 & -247.7 & -289.1 & -158.0 & -126.0 & -164.4 & -159.8 & -354.7 & -315.5 \\ 463.3 & 560.2 & 637.5 & 679.4 & 733.4 & 788.2 & 837.8 & 951.8 & 1,066.1 \\ 0.0 & 0.0 & 0.0 & 0.0 & 0.0 & 0.0 & 0.0 & 0.0 & 0.0 \\ 0.0 & 0.0 & 0.0 & 0.0 & 0.0 & 0.0 & 0.0 & 0.0 & 0.0 \\ 463.3 & 560.2 & 637.5 & 679.4 & 733.4 & 788.2 & 837.8 & 951.8 & 1,066.1 \\ 77.7 & 96.9 & 77.3 & 41.9 & 53.9 & 54.8 & 49.6 & 114.0 & 114.3 \\ 0.1429 & 0.3255 & 0.3781 & 0.1822 & 0.1060 & 0.1494 & 0.1398 & 0.2874 & 0.2114 \\ 55.1 & 150.8 & 211.8 & 116.1 & 72.0 & 109.6 & 110.2 & 240.8 & 201.2 \\ & & & & & & & & \\ 99.7 & 191.1 & 229.1 & 122.6 & 90.0 & 122.9 & 115.2 & 263.5 & 232.6 \\ 0.5 & 0.7 & 0.5 & 0.2 & 0.0 & 0.0 & 0.1 & 0.1 & 0.4 \\ -99.2 & -190.4 & -228.5 & -122.4 & -89.9 & -122.8 & -115.1 & -263.4 & -232.2 \\ 376.0 & 432.6 & 487.0 & 526.6 & 552.1 & 593.3 & 619.5 & 692.6 & 788.9 \\ 0.0 & 0.0 & 0.0 & 0.0 & 0.0 & 0.0 & 0.0 & 0.0 & 0.0 \\ 0.0 & 0.0 & 0.0 & 0.0 & 0.0 & 0.0 & 0.0 & 0.0 & 0.0 \\ 376.0 & 432.6 & 487.0 & 526.6 & 552.1 & 593.3 & 619.5 & 692.6 & 788.9 \\ 48.5 & 56.5 & 54.4 & 39.7 & 25.5 & 41.1 & 26.2 & 73.1 & 96.4 \\ 0.1549 & 0.3561 & 0.4025 & 0.1700 & 0.1223 & 0.1480 & 0.1498 & 0.3071 & 0.1961 \\ 50.7 & 133.9 & 174.1 & 82.8 & 64.4 & 81.7 & 88.9 & 190.3 & 135.8 \\ 0.3807 & 0.4598 & 0.4724 & 0.3913 & 0.3781 & 0.3744 & 0.3391 & 0.4340 & 0.4260 \\ & & & & & & & & \\ 61.0 & 80.6 & 135.5 & 109.1 & 70.6 & 98.5 & 101.6 & 147.1 & 148.6 \\ 0.0 & 0.0 & 0.0 & 0.0 & 0.0 & 0.0 & 0.0 & 0.0 & 0.0 \\ -61.0 & -80.6 & -135.5 & -109.1 & -70.6 & -98.5 & -101.6 & -147.1 & -148.6 \\ 413.0 & 408.0 & 402.7 & 439.4 & 460.1 & 492.9 & 522.5 & 522.1 & 564.9 \\ 0.0 & 0.0 & 0.0 & 0.0 & 0.0 & 0.0 & 0.0 & 0.0 & 0.0 \\ 0.0 & 0.0 & 0.0 & 0.0 & 0.0 & 0.0 & 0.0 & 0.0 & 0.0 \\ 413.0 & 408.0 & 402.7 & 439.4 & 460.1 & 492.9 & 522.5 & 522.1 & 564.9 \\ 21.8 & -5.0 & -5.3 & 36.7 & 20.7 & 32.8 & 29.7 & -0.5 & 42.9 \\ 0.1001 & 0.2073 & 0.3451 & 0.1798 & 0.1136 & 0.1428 & 0.1460 & 0.2823 & 0.2025 \\ 39.2 & 85.6 & 140.8 & 72.4 & 49.9 & 65.7 & 72.0 & 147.5 & 105.7 \\ 0.9692 & 1.0976 & 1.1221 & 0.7732 & 1.0194 & 0.9380 & 1.0051 & 1.0091 & 0.8646\end{array}$


Table 1

Estimates of Mortgage Originations Derived from Mortgage Debt Outstanding and Repayments

(billions of dollars, except where noted; levels are end-of-year values, except where noted)

\begin{tabular}{|c|c|c|c|c|c|c|c|c|c|}
\hline & & \multirow[b]{2}{*}{2000} & \multirow[b]{2}{*}{2001} & \multirow[b]{2}{*}{2002} & \multirow[b]{2}{*}{2003} & \multirow[b]{2}{*}{2004} & \multicolumn{3}{|c|}{ Average } \\
\hline & & & & & & & $\begin{array}{r}1991- \\
1992\end{array}$ & $\begin{array}{r}1993- \\
2004\end{array}$ & $\begin{array}{r}1991- \\
2004\end{array}$ \\
\hline \multicolumn{10}{|c|}{ The GSEs and Ginnie Mae } \\
\hline (245) & Purchases & 227.1 & 567.7 & 800.3 & $1,322.0$ & 587.9 & 191.1 & 422.9 & 389.8 \\
\hline$(246)$ & Sales & 1.1 & 0.9 & 1.0 & 1.4 & 1.6 & 0.8 & 0.7 & 0.7 \\
\hline (247) & Net sales [(246)-(245)] & -226.0 & -566.8 & -799.4 & $-1,320.7$ & -586.3 & -190.3 & -422.2 & -389.1 \\
\hline (248) & 1-4 MDO, end of quarter (held in porfolio or in securitized pools) & $1,160.5$ & $1,393.5$ & $1,650.8$ & $2,000.8$ & $2,042.1$ & 511.7 & $1,161.8$ & $1,069.0$ \\
\hline (249) & Construction loans & 0.0 & 0.0 & 0.0 & 0.0 & 0.0 & 0.0 & 0.0 & 0.0 \\
\hline (250) & Home equity loans & 0.0 & 0.0 & 0.0 & 0.0 & 0.0 & 0.0 & 0.0 & 0.0 \\
\hline (251) & Regular mortgages [(248)-(249)-(250)] & $1,160.5$ & $1,393.5$ & $1,650.8$ & $2,000.8$ & $2,042.1$ & 511.7 & $1,161.8$ & $1,069.0$ \\
\hline (252) & Change & 94.4 & 233.0 & 257.3 & 349.9 & 41.3 & 87.3 & 123.5 & 118.3 \\
\hline (253) & Repayment rate $[(254) /(251)\{t-1\}]$ & 0.1234 & 0.2876 & 0.3890 & 0.5880 & 0.2724 & 0.2342 & 0.2596 & 0.2559 \\
\hline (254) & Repayments [(245)-(246)-(252)] & 131.6 & 333.8 & 542.1 & 970.7 & 545.0 & 102.9 & 298.7 & 270.8 \\
\hline \multicolumn{10}{|c|}{ Freddie Mac } \\
\hline$(255)$ & Purchases & 168.0 & 393.2 & 547.7 & 701.5 & 354.8 & 145.4 & 278.4 & 259.4 \\
\hline (256) & Sales & 0.8 & 0.6 & 0.7 & 0.8 & 1.0 & 0.6 & 0.4 & 0.5 \\
\hline (257) & Net sales [(256)-(255)] & -167.2 & -392.5 & -547.0 & -700.7 & -353.8 & -144.8 & -278.0 & -259.0 \\
\hline (258) & 1-4 MDO, end of quarter (held in porfolio or in securitized pools) & 859.5 & 981.2 & $1,108.8$ & $1,168.5$ & $1,215.5$ & 404.3 & 799.5 & 743.0 \\
\hline (259) & Construction loans & 0.0 & 0.0 & 0.0 & 0.0 & 0.0 & 0.0 & 0.0 & 0.0 \\
\hline (260) & Home equity loans & 0.0 & 0.0 & 0.0 & 0.0 & 0.0 & 0.0 & 0.0 & 0.0 \\
\hline (261) & Regular mortgages [(258)-(259)-(260)] & 859.5 & 981.2 & $1,108.8$ & $1,168.5$ & $1,215.5$ & 404.3 & 799.5 & 743.0 \\
\hline$(262)$ & Change & 70.5 & 121.8 & 127.6 & 59.7 & 47.0 & 52.5 & 65.2 & 63.4 \\
\hline (263) & Repayment rate $[(264) /(261)\{t-1\}]$ & 0.1226 & 0.3151 & 0.4275 & 0.5858 & 0.2588 & 0.2555 & 0.2671 & 0.2655 \\
\hline$(264)$ & Repayments [(255)-(256)-(262)] & 96.7 & 270.8 & 419.4 & 641.0 & 306.8 & 92.3 & 212.7 & 195.5 \\
\hline \multicolumn{10}{|c|}{ Ginnie Mae } \\
\hline$(265)$ & Purchases (issuance) & 100.4 & 168.8 & 166.3 & 205.6 & 94.4 & 70.8 & 128.9 & 120.6 \\
\hline (266) & Sales & 0.0 & 0.0 & 0.0 & 0.0 & 0.0 & 0.0 & 0.0 & 0.0 \\
\hline (267) & Net sales [(266)-(265)] & -100.4 & -168.8 & -166.3 & -205.6 & -94.4 & -70.8 & -128.9 & -120.6 \\
\hline (268) & 1-4 MDO, end of quarter (securitized pools) & 592.5 & 567.6 & 512.0 & 444.8 & 409.2 & 410.5 & 494.2 & 482.3 \\
\hline (269) & Construction loans & 0.0 & 0.0 & 0.0 & 0.0 & 0.0 & 0.0 & 0.0 & 0.0 \\
\hline$(270)$ & Home equity loans & 0.0 & 0.0 & 0.0 & 0.0 & 0.0 & 0.0 & 0.0 & 0.0 \\
\hline (271) & Regular mortgages [(268)-(269)-(270)] & 592.5 & 567.6 & 512.0 & 444.8 & 409.2 & 410.5 & 494.2 & 482.3 \\
\hline (272) & Change & 27.6 & -25.0 & -55.5 & -67.2 & -35.6 & 8.4 & 0.1 & 1.3 \\
\hline (273) & Repayment rate $[(274) /(271)\{t-1\}]$ & 0.1289 & 0.3270 & 0.3908 & 0.5328 & 0.2924 & 0.1537 & 0.2570 & 0.2423 \\
\hline (274) & Repayments [(265)-(266)-(272)] & 72.8 & 193.8 & 221.8 & 272.8 & 130.1 & 62.4 & 128.8 & 119.3 \\
\hline (275) & GNM purchases / (VA and FHA originations) & 0.8793 & 1.0000 & 0.8893 & 0.8882 & 0.7323 & 1.0334 & 0.9267 & 0.9420 \\
\hline
\end{tabular}


Table 1

Estimates of Mortgage Originations Derived from Mortgage Debt Outstanding and Repayments

(billions of dollars, except where noted; levels are end-of-year values, except where noted)

1991

1992

1993

1994

1995

1996

1997

1998

1999

The GSEs and Ginnie Mae

Federal Home Loan Banks

(276) Purchases

(277) Sales

(278) Net sales [(277)-(276)]

(279) 1-4 mortgage debt outstanding, end of quarter

(280) Construction loans

(281) Home equity loans

(282) Regular mortgages [(279)-(280)-(281)]

(283) Change

(284) Repayment rate $[(285) /(282)\{t-1\}]$

(285) Repayments [(276)-(277)-(283)]

\section{GSEs and Ginnie Mae}

(286) Purchases [(245)+(255)+(265)+(276)]]

(287) Excluding Federal Home Loan Banks

(288) Sales $[(246)+(256)+(266)+(277)]]$

(289) Net sales [(288)-(286)]

(290) 1-4 MDO, end of quarter (held in porfolio or in securitized pools)

(291) Construction loans

(292) Home equity loans

(293) Regular mortgages [(290)-(291)-(292)]

(294) Change

(295) Repayment rate $[(296) /(293)\{t-1\}]$

(296) Repayments [(286)-(288)-(294)]

(297) Repayments net of refinancings $\left[(293)^{\star}(93)\{t-1\}\right]$ (sum of quarterly)

(298) Repayment rate net of refinancings $[(297) /(293)\{t-1\})]$

\section{Private MBS}

(299) Non-agency MBS excluding subprime and second liens

(300) Subprime

(301) Home equity loans

(302) Non-agency MBS regular mortgages [(299)+(300)]

(303) Sales

(304) Net sales [ $-1 *(302)]$

(305) 1-4 mortgage debt outstanding, end of quarter"

(306) Construction loans

(307) Home equity loans

(308) Regular mortgage debt [(305)-(306)-(307)]

(309) Change $[(308)-(308)\{t-1\})]$

(310) Repayments $[(-1) \star\{(309)+(304)\}]$

(311) Repayment rate $[(310) /(308)\{t-1\}]$

(312) Repayments net of refinancings [(307)*(93)\{t-1\}] (sum of quarterly)

(314) Repayment rate net of refinancings $[(312) /(307)\{t-1\})]$

0.0
0.0
0.0
0.0
0.0000

\section{0}

0.0

0.0

0.0

0.0

0.0

0.0

0.0

0.0000

0.0

0.0
0.0

0.0

0.0
0.0

0.0
0.0

0.0
0.0

0.0000

0000
0.0

$294.3 \quad 520.3$

294.3

1.2

$-293.0$

$1,252.3$

520.3
1.6

-518.7
1.400 .7

$1,400.7$

0.0
0.0

$1,252.3$

148.0

0.1313

145.0

110.1

0.0997

0.0
0.0
$1,400.7$
148.4

0.2957

370.3

112.3

0.0897

$\begin{array}{rr}49.4 & 89.5 \\ 1.5 & 2.8 \\ 0.6 & 0.9 \\ 57.7 & 92.2 \\ 0.0 & 0.0 \\ -57.7 & -92.2 \\ 98.2 & 146.1 \\ 0.0 & 0.0 \\ 0.4 & 1.1 \\ 97.7 & 145.0 \\ 42.6 & 47.3\end{array}$

0.0
0.0
0.0
0.0
0.0
0.0
0.0
0.0000

0.0
0.0
0.0
0.0
0.0
0.0
0.0
0.0
0.0000
0.0

0.0
0.0
0.0
0.0
0.0
0.0
0.0
0.0
0.0000
0.0

0.0
0.0
0.0
0.0
0.0
0.0
0.0
0.0
0.0000
0.0

0.0

0.0
0.0

$$
\begin{aligned}
& 0.0 \\
& 0.0
\end{aligned}
$$$$
0.0
$$$$
\begin{aligned}
& 0.0 \\
& 0.0
\end{aligned}
$$$$
\begin{aligned}
& 0.0 \\
& 0.0
\end{aligned}
$$$$
\begin{aligned}
& 0.0 \\
& 0.0
\end{aligned}
$$$$
0.0
$$

0.0000

0.0

0.0

0.0
0.0
0.0

0.0

1.0
0.0

0.0
0.0

1.0

0.0000
-0.9

$654.4 \quad 389.9$

286.6

654.4

389.9
389.9

$-653.1$

1,527.2

0.3
-389.6

$1,645.4$

286.6

0.1
-286.5

$1,745.6$

385.8
385.8
0.0
-385.8
$1,874.3$

0.0
0.0
$1,527.2$

0.0

$1,527.2$
126.4

0.3760

$1,645.4$

118.3

0.0
0.0

1,745.6

100.2

0.1132

0.1777

134.5

0.0961

0.1074

186.3

142.0
0.0863

15.1

0.2736

45.0
0.4601

0.4601
9.9

0.1164

0.1014
98.5

5.2
1.0
103.7

1.0
103.7

103.7
0.0

$-103.7$

174.4

0.0
1.8

1.8
172.7

172.7
27.7

76.0
0.5243

0.5243
14.4

0.0995
63.2

11.1
1.5

1.5
74.2

0.0

$-74.2$

192.4

0.0
2.5

189.9

17.3

29.1

17.8
2.0

46.9

0.0

$-46.9$

206.2

0.0
3.3

202.9

13.0

33.9
0.1786

0.3300

19.0

0.1786

16.6
0.0873
376.7

376.7

0.2

$-376.5$

$1,979.8$

0.0

0.0
$1,874.3$

128.8
0.874 .3

0.1472

257.0

183.6

0.1052

0.0

1,979.8

105.5

0.1446

271.0

188.3

0.1005

\section{5}

765.5

0.3
-765.2

2,167.4

0.0

0.0
0.0
$2,167.4$

187.6

0.2917

577.6
191.6

57.6
0.0968

0.0968

34.0

57.6

120.0

56.9
4.6

30.8
5.1
64.8
0.0

0.0

231.8

0.0

4.4
227.5

227.5
24.6

40.2

0.1981

22.0

0.1085

114.6
0.0

-114.6
276.4

0.0

6.1

270.3

42.9

75.8
7.4

195.8

0.0

$-195.8$

-195.8
354.7

345.9

75.5

71.7
0.3152

120.3
0.4451

239

0.4451

0.1052

0.1040
$526.7 \quad 271.3$

39.3

0.1136 
Table 1

Estimates of Mortgage Originations Derived from Mortgage Debt Outstanding and Repayments

(billions of dollars, except where noted; levels are end-of-year values, except where noted)

\begin{tabular}{|c|c|c|c|c|c|c|c|c|c|}
\hline & & \multirow[b]{2}{*}{2000} & \multirow[b]{2}{*}{2001} & \multirow[b]{2}{*}{2002} & \multirow[b]{2}{*}{2003} & \multirow[b]{2}{*}{2004} & \multicolumn{3}{|c|}{ Average } \\
\hline & & & & & & & $\begin{array}{r}1991- \\
1992 \\
\end{array}$ & $\begin{array}{r}1993- \\
2004 \\
\end{array}$ & $\begin{array}{r}1991- \\
2004 \\
\end{array}$ \\
\hline \multicolumn{10}{|c|}{ The GSEs and Ginnie Mae } \\
\hline (276) & Purchases & 14.6 & 19.4 & 45.7 & 91.2 & 24.7 & & & 39.1 \\
\hline (277) & Sales & 0.0 & 0.0 & 0.0 & 0.0 & 0.0 & & & 0.0 \\
\hline (278) & Net sales [(277)-(276)] & -14.6 & -19.4 & -45.7 & -91.2 & -24.7 & & & -39.1 \\
\hline (279) & 1-4 mortgage debt outstanding, end of quarter & 15.9 & 27.5 & 60.3 & 113.3 & 113.8 & & & 66.2 \\
\hline (280) & Construction loans & 0.0 & 0.0 & 0.0 & 0.0 & 0.0 & & & 0.0 \\
\hline (281) & Home equity loans & 0.0 & 0.0 & 0.0 & 0.0 & 0.0 & & & 0.0 \\
\hline (282) & Regular mortgages [(279)-(280)-(281)] & 15.9 & 27.5 & 60.3 & 113.3 & 113.8 & & & 66.2 \\
\hline (283) & Change & 14.1 & 11.6 & 32.8 & 53.0 & 0.6 & & & 22.4 \\
\hline (284) & Repayment rate $[(285) /(282)\{\mathrm{t}-1\}]$ & 0.2589 & 0.4938 & 0.4700 & 0.6338 & 0.2133 & & & 0.4139 \\
\hline$(285)$ & Repayments [(276)-(277)-(283)] & 0.5 & 7.9 & 12.9 & 38.2 & 24.2 & & & 16.7 \\
\hline \multicolumn{10}{|c|}{ GSEs and Ginnie Mae } \\
\hline (286) & Purchases [(245)+(255)+(265)+(276)]] & 510.1 & $1,149.0$ & $1,560.0$ & $2,320.3$ & $1,061.9$ & 407.3 & 846.6 & 783.8 \\
\hline (287) & Excluding Federal Home Loan Banks & 495.5 & $1,129.6$ & $1,514.3$ & $2,229.1$ & $1,037.2$ & 407.3 & 830.2 & 769.7 \\
\hline (288) & Sales [(246)+(256)+(266)+(277)]] & 1.9 & 1.5 & 1.6 & 2.1 & 2.6 & 1.4 & 1.1 & 1.1 \\
\hline (289) & Net sales [(288)-(286)] & -508.2 & $-1,147.5$ & $-1,558.4$ & $-2,318.1$ & $-1,059.3$ & -405.9 & -845.5 & -782.7 \\
\hline (290) & 1-4 MDO, end of quarter (held in porfolio or in securitized pools) & $2,628.5$ & $2,969.8$ & $3,332.0$ & $3,727.4$ & $3,780.6$ & $1,326.5$ & $2,483.3$ & $2,318.1$ \\
\hline (291) & Construction loans & 0.0 & 0.0 & 0.0 & 0.0 & 0.0 & 0.0 & 0.0 & 0.0 \\
\hline (292) & Home equity loans & 0.0 & 0.0 & 0.0 & 0.0 & 0.0 & 0.0 & 0.0 & 0.0 \\
\hline (293) & Regular mortgages [(290)-(291)-(292)] & $2,628.5$ & $2,969.8$ & $3,332.0$ & $3,727.4$ & $3,780.6$ & $1,326.5$ & $2,483.3$ & $2,318.1$ \\
\hline (294) & Change & 206.7 & 341.3 & 362.2 & 395.3 & 53.2 & 148.2 & 198.3 & 191.2 \\
\hline (295) & Repayment rate $[(296) /(293)\{t-1\}]$ & 0.1245 & 0.3067 & 0.4028 & 0.5771 & 0.2699 & 0.2135 & 0.2613 & 0.2545 \\
\hline (296) & Repayments [(286)-(288)-(294)] & 301.6 & 806.2 & $1,196.2$ & $1,922.8$ & $1,006.0$ & 257.6 & 647.2 & 591.5 \\
\hline (297) & Repayments net of refinancings $\left[(293)^{\star}(93)\{t-1\}\right]$ (sum of quarterly) & 298.8 & 299.9 & 304.0 & 423.4 & 426.6 & 111.2 & 250.2 & 230.4 \\
\hline (298) & Repayment rate net of refinancings [(297)/(293)\{t-1\})] & 0.1234 & 0.1141 & 0.1024 & 0.1271 & 0.1145 & 0.0947 & 0.1073 & 0.1055 \\
\hline \multicolumn{10}{|c|}{ Private MBS } \\
\hline (299) & Non-agency MBS excluding subprime and second liens & 74.2 & 164.8 & 266.5 & 370.9 & na & 69.4 & 124.3 & 115.9 \\
\hline (300) & Subprime & 48.1 & 87.1 & 122.7 & 195.0 & na & 2.1 & 64.2 & 54.7 \\
\hline (301) & Home equity loans & 3.8 & 15.5 & 24.8 & 20.4 & na & 0.8 & 8.1 & 7.0 \\
\hline (302) & Non-agency MBS regular mortgages [(299)+(300)] & 122.3 & 251.8 & 389.2 & 565.9 & 815.0 & 75.0 & 240.7 & 217.1 \\
\hline (303) & Sales & 0.0 & 0.0 & 0.0 & 0.0 & 0.0 & 0.0 & 0.0 & 0.0 \\
\hline (304) & Net sales [ $-1 *(302)]$ & -122.3 & -251.8 & -389.2 & -565.9 & -815.0 & -75.0 & -240.7 & -217.1 \\
\hline (305) & 1-4 mortgage debt outstanding, end of quarter" & 425.1 & 494.7 & 560.5 & 698.9 & $1,076.4$ & 122.1 & 423.8 & 380.7 \\
\hline (306) & Construction loans & 0.0 & 0.0 & 0.0 & 0.0 & 0.0 & 0.0 & 0.0 & 0.0 \\
\hline (307) & Home equity loans & 13.0 & 16.5 & 18.8 & 19.6 & 27.2 & 0.7 & 11.1 & 9.6 \\
\hline (308) & Regular mortgage debt [(305)-(306)-(307)] & 412.1 & 478.1 & 541.7 & 679.2 & $1,049.2$ & 121.4 & 412.7 & 371.1 \\
\hline (309) & Change $[(308)-(308)\{t-1\})]$ & 29.4 & 66.0 & 63.6 & 137.5 & 369.9 & 44.9 & 75.3 & 71.0 \\
\hline (310) & Repayments $[(-1) \star\{(309)+(304)\}]]$ & 92.9 & 185.8 & 325.6 & 428.3 & 445.1 & 30.0 & 165.4 & 146.0 \\
\hline (311) & Repayment rate $[(310) /(308)\{t-1\}]$ & 0.2427 & 0.4508 & 0.6809 & 0.7907 & 0.6553 & 0.3668 & 0.4270 & 0.4184 \\
\hline (312) & Repayments net of refinancings $\left[(307)^{\star}(93)\{t-1\}\right]$ (sum of quarterly) & 47.2 & 47.5 & 49.0 & 71.8 & 92.3 & 8.2 & 39.3 & 34.8 \\
\hline (314) & Repayment rate net of refinancings [(312)/(307)\{t-1\})] & 0.1233 & 0.1152 & 0.1026 & 0.1326 & 0.1359 & 0.1089 & 0.1115 & 0.1111 \\
\hline
\end{tabular}


Table 1

Estimates of Mortgage Originations Derived from Mortgage Debt Outstanding and Repayments

(billions of dollars, except where noted; levels are end-of-year values, except where noted)

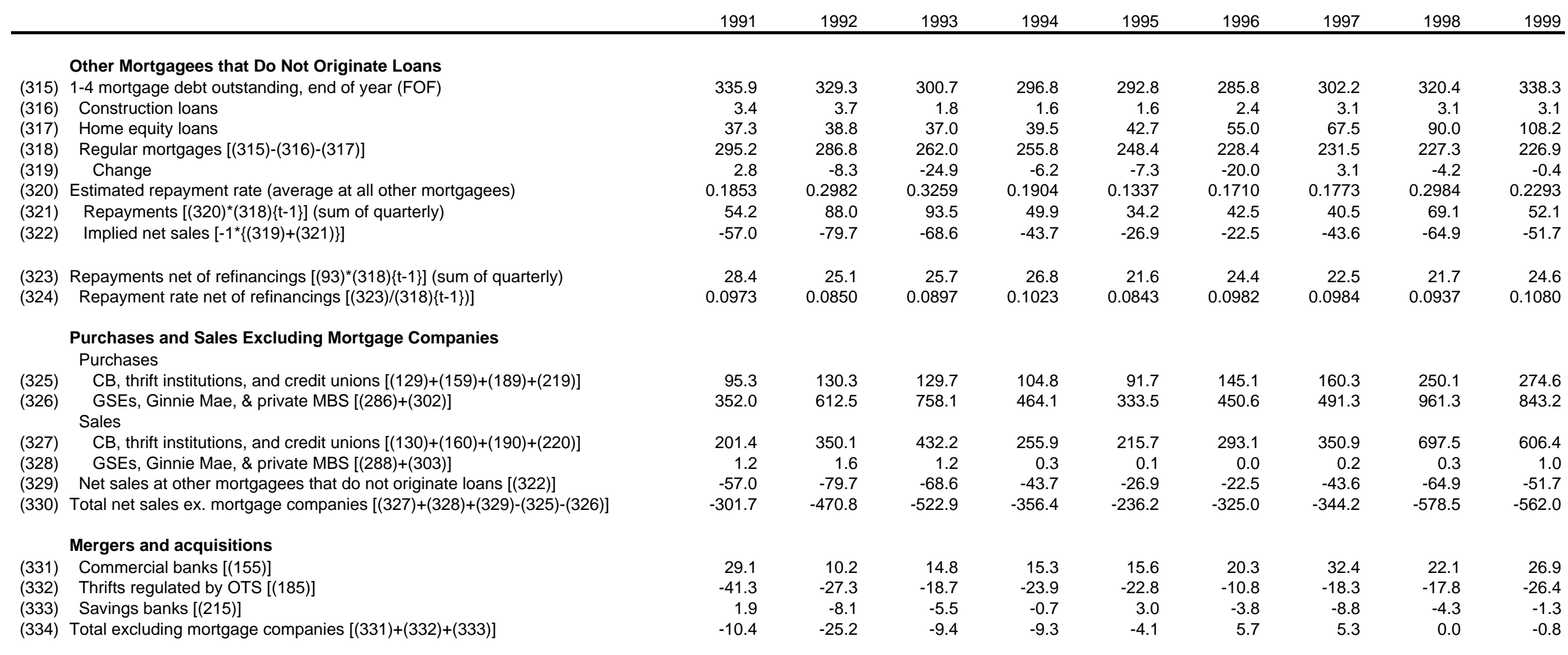


Table 1

Estimates of Mortgage Originations Derived from Mortgage Debt Outstanding and Repayments (billions of dollars, except where noted; levels are end-of-year values, except where noted)

\begin{tabular}{|c|c|c|c|c|c|c|c|c|c|}
\hline & & & & & & & \multicolumn{3}{|c|}{ Average } \\
\hline & & 2000 & 2001 & 2002 & 2003 & 2004 & $\begin{array}{r}1991- \\
1992 \\
\end{array}$ & $\begin{array}{r}1993- \\
2004 \\
\end{array}$ & $\begin{array}{r}1991- \\
2004 \\
\end{array}$ \\
\hline \multicolumn{10}{|c|}{ Other Mortgagees that Do Not Originate Loans } \\
\hline (315) & 1-4 mortgage debt outstanding, end of year (FOF) & 362.2 & 359.8 & 390.1 & 439.2 & 548.3 & 332.6 & 353.0 & 350.1 \\
\hline (316) & Construction loans & 3.1 & 3.1 & 3.1 & 3.1 & 3.1 & 3.6 & 2.7 & 2.8 \\
\hline (317) & Home equity loans & 130.6 & 120.1 & 135.0 & 152.2 & 190.2 & 38.1 & 97.3 & 88.9 \\
\hline (318) & Regular mortgages [(315)-(316)-(317)] & 228.4 & 236.5 & 252.0 & 283.8 & 355.0 & 291.0 & 253.0 & 258.4 \\
\hline (319) & Change & 1.5 & 8.1 & 15.5 & 31.9 & 71.2 & -2.8 & 5.7 & 4.5 \\
\hline (320) & Estimated repayment rate (average at all other mortgagees) & 0.1708 & 0.3526 & 0.4226 & 0.5808 & 0.3284 & 0.2417 & 0.2818 & 0.2760 \\
\hline (321) & Repayments [(320**(318)\{t-1\}] (sum of quarterly) & 38.8 & 80.5 & 100.0 & 146.3 & 93.2 & 71.1 & 70.0 & 70.2 \\
\hline (322) & Implied net sales $[-1 *\{(319)+(321)\}]$ & -40.3 & -88.6 & -115.4 & -178.2 & -164.4 & -68.3 & -75.7 & -74.7 \\
\hline (323) & Repayments net of refinancings [(93)^(318)\{t-1\}] (sum of quarterly) & 27.2 & 25.3 & 23.3 & 31.7 & 34.9 & 26.8 & 25.8 & 25.9 \\
\hline (324) & Repayment rate net of refinancings $[(323) /(318)\{t-1\})]$ & 0.1201 & 0.1106 & 0.0986 & 0.1258 & 0.1231 & 0.0912 & 0.1044 & 0.1025 \\
\hline \multicolumn{10}{|c|}{$\begin{array}{l}\text { Purchases and Sales Excluding Mortgage Companies } \\
\text { Purchases }\end{array}$} \\
\hline (325) & CB, thrift institutions, and credit unions $[(129)+(159)+(189)+(219)]$ & 210.4 & 401.6 & 551.1 & 857.9 & 549.0 & 112.8 & 310.5 & 282.3 \\
\hline (326) & $\begin{array}{l}\text { GSEs, Ginnie Mae, \& private MBS }[(286)+(302)] \\
\text { Sales }\end{array}$ & 632.4 & $1,400.9$ & $1,949.2$ & $2,886.2$ & $1,876.9$ & 482.2 & $1,087.3$ & $1,000.9$ \\
\hline$(327)$ & CB, thrift institutions, and credit unions $[(130)+(160)+(190)+(220)]$ & 481.3 & $1,111.1$ & $1,556.4$ & $2,371.4$ & $1,381.5$ & 275.8 & 812.8 & 736.1 \\
\hline (328) & GSEs, Ginnie Mae, \& private MBS [(288)+(303)] & 1.9 & 1.5 & 1.6 & 2.1 & 2.6 & 1.4 & 1.1 & 1.1 \\
\hline (329) & Net sales at other mortgagees that do not originate loans [(322)] & -40.3 & -88.6 & -115.4 & -178.2 & -164.4 & -68.3 & -75.7 & -74.7 \\
\hline (330) & Total net sales ex. mortgage companies $[(327)+(328)+(329)-(325)-(326)]$ & -399.9 & -778.5 & $-1,057.7$ & $-1,548.7$ & $-1,206.2$ & -386.2 & -659.7 & -620.6 \\
\hline \multicolumn{10}{|c|}{ Mergers and acquisitions } \\
\hline (331) & Commercial banks [(155)] & 32.2 & 23.1 & 8.1 & 3.3 & 4.0 & 19.7 & 18.2 & 18.4 \\
\hline (332) & Thrifts regulated by OTS [(185)] & -5.5 & -21.8 & -0.7 & 1.2 & 46.1 & -34.3 & -10.0 & -13.4 \\
\hline (333) & Savings banks $[(215)]$ & -8.1 & -1.7 & -3.3 & -2.8 & -24.6 & -3.1 & -5.2 & -4.9 \\
\hline (334) & Total excluding mortgage companies $[(331)+(332)+(333)]$ & 18.6 & -0.4 & 4.1 & 1.6 & 25.5 & -17.8 & 3.1 & 0.1 \\
\hline
\end{tabular}


Table 1

Estimates of Mortgage Originations Derived from Mortgage Debt Outstanding and Repayments

(billions of dollars, except where noted; levels are end-of-year values, except where noted)

\begin{tabular}{|c|c|c|c|c|c|c|c|c|c|c|}
\hline & & 1991 & 1992 & 1993 & 1994 & 1995 & 1996 & 1997 & 1998 & 1999 \\
\hline & Mortgage Companies & & & & & & & & & \\
\hline (335) & Implied originations [(336)+(338)+(340)-(341)] & 314.4 & 462.6 & 526.8 & 331.4 & 232.6 & 346.2 & 346.6 & 585.1 & 567.2 \\
\hline (336) & Implied net sales $\left[-1^{*}(330)\right]$ & 301.7 & 470.8 & 522.9 & 356.4 & 236.2 & 325.0 & 344.2 & 578.5 & 562.0 \\
\hline (337) & Regular mortgage debt outstanding (FOF) & 53.1 & 52.7 & 52.2 & 28.3 & 23.8 & 32.0 & 21.8 & 21.8 & 21.8 \\
\hline (338) & Change & 11.3 & -0.4 & -0.5 & -24.0 & -4.5 & 8.2 & -10.2 & 0.0 & 0.0 \\
\hline (339) & Repayment rate (equal to the average at depository institutions) & 0.2869 & 0.3271 & 0.2607 & 0.1591 & 0.1804 & 0.3090 & 0.2270 & 0.3039 & 0.2732 \\
\hline (340) & Repayments $\left[(339)^{\star}(337)\{\mathrm{t}-1\}\right]$ (sum of quarterly) & 11.9 & 17.4 & 13.7 & 8.3 & 5.1 & 7.3 & 7.3 & 6.6 & 6.0 \\
\hline (341) & Mergers and acquisitions $\left[(-1)^{\star}(334)\right]$ & 10.4 & 25.2 & 9.4 & 9.3 & 4.1 & -5.7 & -5.3 & 0.0 & 0.8 \\
\hline (342) & HMDA: Refinance share of originations & 0.3341 & 0.5960 & 0.6588 & 0.4166 & 0.2962 & 0.3505 & 0.3896 & 0.5547 & 0.4148 \\
\hline (343) & Purchase originations [(335)-(344)\}] & 209.4 & 186.9 & 179.8 & 193.3 & 163.7 & 224.9 & 211.6 & 260.6 & 331.9 \\
\hline (344) & Refinance originations (sum of quarterly) $\left[(342)^{\star}(335)\right]$ & 105.0 & 275.7 & 347.0 & 138.1 & 68.9 & 121.3 & 135.0 & 324.6 & 235.2 \\
\hline (345) & Percent to payoff retired loans [1-(125)] & 0.8673 & 0.9276 & 0.9244 & 0.8711 & 0.8654 & 0.8499 & 0.8540 & 0.8973 & 0.8426 \\
\hline (346) & Repayments net of refis $\left[(93)^{\star}(337)\{t-1\}\right]$ (sum of quarterly) & 4.5 & 4.7 & 4.8 & 4.0 & 2.5 & 3.2 & 3.3 & 2.0 & 2.4 \\
\hline (347) & Repayment rate net of refinancings $[(346) /(337)\{t-1\})]$ & 0.1094 & 0.0892 & 0.0910 & 0.0768 & 0.0895 & 0.1347 & 0.1037 & 0.0938 & 0.1080 \\
\hline (348) & HMDA originations & 134.6 & 236.1 & 466.3 & 242.7 & 187.4 & 234.5 & 275.9 & 505.1 & 394.9 \\
\hline (349) & HMDA originations / total originations at mortgage companies $[(348) /(335)]$ & 0.4280 & 0.5105 & 0.8852 & 0.7323 & 0.8055 & 0.6774 & 0.7961 & 0.8633 & 0.6963 \\
\hline (350) & HMDA Purchases & 40.0 & 83.6 & 78.6 & 53.0 & 54.6 & 73.3 & 86.7 & 215.4 & 112.9 \\
\hline (351) & HMDA Sales & 145.7 & 275.7 & 450.6 & 243.0 & 181.8 & 241.1 & 301.8 & 634.2 & 434.2 \\
\hline (352) & Adjusted and temporally reallocated & 150.2 & 282.5 & 467.4 & 282.1 & 187.8 & 255.2 & 311.5 & 649.1 & 487.3 \\
\hline (353) & Raw HMDA sales / adjusted HMDA sales [(351)/(352)] & 0.9700 & 0.9762 & 0.9642 & 0.8612 & 0.9682 & 0.9450 & 0.9688 & 0.9771 & 0.8910 \\
\hline (354) & Adjusted HMDA net sales [(352)-(350)] & 110.1 & 198.9 & 388.8 & 229.1 & 133.2 & 181.9 & 224.8 & 433.7 & 374.4 \\
\hline (355) & Sales / (originations + purchases) $[(352) /\{(348)+(350)\}]$ & 0.8602 & 0.8834 & 0.8578 & 0.9541 & 0.7759 & 0.8289 & 0.8589 & 0.9009 & 0.9595 \\
\hline
\end{tabular}


Table 1

Estimates of Mortgage Originations Derived from Mortgage Debt Outstanding and Repayments (billions of dollars, except where noted; levels are end-of-year values, except where noted)

\begin{tabular}{|c|c|c|c|c|c|c|c|c|c|}
\hline & & & & & & & \multicolumn{3}{|c|}{ Average } \\
\hline & & 2000 & 2001 & 2002 & 2003 & 2004 & $\begin{array}{r}1991- \\
1992 \\
\end{array}$ & $\begin{array}{r}1993- \\
2004 \\
\end{array}$ & $\begin{array}{r}1991- \\
2004 \\
\end{array}$ \\
\hline & Mortgage Companies & & & & & & & & \\
\hline (335) & Implied originations [(336)+(338)+(340)-(341)] & 424.0 & 787.8 & $1,070.9$ & $1,561.8$ & $1,237.6$ & 388.5 & 668.2 & 628.2 \\
\hline (336) & Implied net sales $\left[-1^{*}(330)\right]$ & 399.9 & 778.5 & $1,057.7$ & $1,548.7$ & $1,206.2$ & 386.2 & 659.7 & 620.6 \\
\hline (337) & Regular mortgage debt outstanding (FOF) & 21.8 & 21.8 & 21.8 & 21.8 & 21.8 & 52.9 & 25.9 & 29.8 \\
\hline (338) & Change & 0.0 & 0.0 & 0.0 & 0.0 & 0.0 & 5.4 & -2.6 & -1.4 \\
\hline (339) & Repayment rate (equal to the average at depository institutions) & 0.2495 & 0.4441 & 0.4186 & 0.5263 & 0.2722 & 0.3070 & 0.3020 & 0.3027 \\
\hline (340) & Repayments $\left[(339)^{\star}(337)\{t-1\}\right]$ (sum of quarterly) & 5.4 & 9.7 & 9.1 & 11.5 & 5.9 & 14.6 & 8.0 & 8.9 \\
\hline (341) & Mergers and acquisitions $\left[(-1)^{\star}(334)\right]$ & -18.6 & 0.4 & -4.1 & -1.6 & -25.5 & 17.8 & -3.1 & -0.1 \\
\hline (342) & HMDA: Refinance share of originations & 0.2805 & 0.5789 & 0.6435 & 0.6817 & 0.5438 & 0.4651 & 0.4841 & 0.4814 \\
\hline (343) & Purchase originations [(335)-(344)\}] & 305.1 & 331.7 & 381.8 & 497.1 & 566.7 & 198.1 & 304.0 & 288.9 \\
\hline (344) & Refinance originations (sum of quarterly) $\left[(342)^{\star}(335)\right]$ & 118.9 & 456.1 & 689.1 & $1,064.7$ & 673.0 & 190.4 & 364.3 & 339.5 \\
\hline (345) & Percent to payoff retired loans [1-(125)] & 0.7463 & 0.8674 & 0.8875 & 0.8980 & 0.8471 & 0.8975 & 0.8626 & 0.8676 \\
\hline (346) & Repayments net of refis $\left[(93)^{\star}(337)\{t-1\}\right]$ (sum of quarterly) & 2.6 & 2.4 & 2.1 & 2.7 & 2.5 & 4.6 & 2.9 & 3.1 \\
\hline (347) & Repayment rate net of refinancings $[(346) /(337)\{t-1\})]$ & 0.1205 & 0.1096 & 0.0978 & 0.1215 & 0.1134 & 0.0993 & 0.1050 & 0.1042 \\
\hline (348) & HMDA originations & 272.4 & 543.0 & 810.2 & $1,078.1$ & 943.0 & 185.3 & 496.1 & 451.7 \\
\hline (349) & HMDA originations / total originations at mortgage companies [(348)/(335)] & 0.6424 & 0.6894 & 0.7565 & 0.6903 & 0.7620 & 0.4692 & 0.7497 & 0.7096 \\
\hline (350) & HMDA Purchases & 91.9 & 164.9 & 295.6 & 475.9 & 337.6 & 61.8 & 170.0 & 154.6 \\
\hline (351) & HMDA Sales & 312.1 & 612.6 & 937.4 & $1,402.6$ & $1,129.6$ & 210.7 & 573.4 & 521.6 \\
\hline (352) & Adjusted and temporally reallocated & 327.8 & 624.9 & 973.9 & $1,492.7$ & 1,190.6 & 216.3 & 604.2 & 548.8 \\
\hline (353) & Raw HMDA sales / adjusted HMDA sales [(351)/(352)] & 0.9523 & 0.9803 & 0.9625 & 0.9396 & 0.9488 & 0.9731 & 0.9466 & 0.9504 \\
\hline (354) & Adjusted HMDA net sales [(352)-(350)] & 235.9 & 460.0 & 678.3 & $1,016.8$ & 853.0 & 154.5 & 434.2 & 394.2 \\
\hline (355) & Sales / (originations + purchases) $[(352) /\{(348)+(350)\}]$ & 0.9000 & 0.8827 & 0.8807 & 0.9606 & 0.9297 & 0.8718 & 0.8908 & 0.8881 \\
\hline
\end{tabular}




\section{Appendix A \\ The Home Mortgage Disclosure Act (HMDA) Data}

HMDA was enacted by the Congress in 1975 and is implemented by the Federal Reserve Board's Regulation C. The survey data may be used to determine whether financial institutions are serving the housing needs of their communities, to assist public officials in distributing public-sector investments to attract private investment to areas where it is needed, and to identify possible discriminatory lending patterns. ${ }^{1}$

HMDA surveys individual lenders. Thus, in the case of a holding company, for example, HMDA requires separate disclosure by the parent and each of its affiliates. HMDA's reporting criteria are listed below.

Lenders report to HMDA information on each application they receive for 1-4 mortgage loans (both home purchase and refinance) and fixed-term home improvement loans. It is optional as to whether lenders report lines of credit used for home improvement. HMDA does not collect information on other types of home equity loans. Respondents also report information on purchases and sales of mortgages. HMDA does not collect data on mortgage debt outstanding or repayments.

\section{A. HMDA's Reporting Criteria}

HMDA has different reporting requirements for depository and non-depository institutions: ${ }^{2}$

\section{Depository Institutions}

1. Is the depository institution a bank, credit union, or savings association?

2. Did the assets of the institution total more than $\$ 32$ million on the preceding December 31?

3. Did the institution have a home or branch office in a metropolitan area (MA) on the preceding December 31 ?

4. In the preceding calendar year, did the institution originate at least one homepurchase loan or refinancing of a home-purchase loan secured by a first lien on a one-to-four-family dwelling?

5. Is the institution federally insured or regulated; or was the mortgage loan insured; guaranteed, or supplemented by a federal agency; or was the loan intended for sale to the Federal National Mortgage Association (FNMA) or Federal Home Loan Mortgage Corporation (FHLMC)?

\footnotetext{
${ }^{1}$ From the FFIEC website, "Home Mortgage Disclosure Act: Background and Purpose."

2 Taken directly from the FFIEC's website, which contains additional information on HMDA's reporting requirements and its evolution over time.
} 
If a depository institution responds 'YES' to the above questions 1 through 4 and 'YES' to at least one question in 5, then HMDA applies to the institution's loan originations, purchases, and applications in the current calendar year. A negative response to any one of the first four questions or to all the questions in 5 would exempt the institution from filing HMDA.

\section{$\underline{\text { Non-Depository Institutions }}$}

1. Is the non-depository institution a for-profit lender?

2. In the preceding calendar year, did the institution's home-purchase loan originations (including refinancings of home-purchase loans) equal or exceed 10 percent of its total loan originations, measured in dollars?

3. Did the non-depository institution either: (a) have a home or branch office in an MA on the preceding December 31, or (b) receive applications for, originate, or purchase 5 or more home-purchase or home improvement loans on property located in an MA in the preceding calendar year?

4. Did the non-depository institution either: (a) have assets (when combined with the assets of any parent corporation) exceeding $\$ 10$ million on the preceding December 31, or (b) originate 100 or more home-purchase loans (including refinancings of home-purchase loans) in the preceding calendar year?

If a non-depository institution responds 'YES' to the above questions 1 and 2, and 'YES' to at least one question in 3 and one question in 4, then HMDA applies to the institution's loan originations, purchases, and applications in the current calendar year. A negative response to either questions 1 or 2, or to all the questions in 3 or 4 exempts the institution from filing HMDA data for the current calendar year.

Thus, in general, HMDA does not cover relatively small lenders or those that operate exclusively outside of MSAs. In addition, because HMDA's reporting thresholds for both mortgage assets and originations pertain to activity in the previous calendar year, the survey excludes lenders that were not in business in the previous year. Furthermore, the HMDA survey excludes mortgage brokers that did not make loan decisions in the previous year, but are doing so in the current year, perhaps because they recently obtained a line of credit.

Even if a lender were to meet the reporting criteria listed above, it would not report to HMDA if HUD had no record of it and if the lender was unaware of HMDA's reporting requirements. HUD has a record of all depository lenders. In addition, federal regulatory authorities have a record of all lenders that sell loans to Fannie Mae or Freddie Mac or that originate FHA or VA-guaranteed loans. Thus, for HUD to have no record of a lender, it would have to be a non-depository institution that did not sell any loans directly to the GSEs and did not originate any FHA or VA-guaranteed loans. 
The entity that makes the credit decision on a mortgage application is required to report that information under HMDA. In the case of retail lending at banks and thrifts, the reporting responsibilities are clear. However, in the case of wholesale or correspondent lending arrangements, mortgage lenders and brokers may not correctly interpret HMDA's reporting requirements. ${ }^{3}$ For example, if a broker receives an application and makes the credit decision, it is responsible for reporting this information, even if it immediately sells the loan to an investor. ${ }^{4}$ However, as Feins, et al note,

“...loans closed in a broker or correspondent's name... [and] acquired by another lender after closing, should be reported by the acquiring lender. On the other hand, if a broker or correspondent makes the credit decision based on an investing lender's underwriting criteria, but the investing lender does not review the loan application prior to closing, the broker or correspondent should report the credit decision..." (page 42)

Given the many types of lending arrangements in the wholesale channel, HMDA's reporting requirements may not always be clear to parties involved. If so, some originations may go unreported to HMDA, and others may be reported twice.

\section{B. Compiling Mortgage Originations, Purchases, and Sales from the HMDA Data}

In order to approximate regular home mortgage flows on residential properties we applied two filters to the HMDA data. The first removes a portion of loans backed by manufactured (mobile) homes and the second attempts to remove home equity loans.

Manufactured homes. HUD provides the Federal Reserve with a list of lenders that primarily originate loans backed by manufactured homes. We assume that the majority of loans made by those companies are personal property loans rather than 1-4 mortgage loans, and thus do not include these lenders. Beginning in 2004, HMDA identifies loans backed by mobile homes, although it does not indicate whether the loan is a 1-4 mortgage loan or a personal property loan. Based on discussions we have had with a people in the industry, we assume that 30 percent of loans backed by mobile homes are 1-4 mortgage loans; the remaining 70 percent are assumed to be personal property loans, and thus are excluded.

Home Equity Loans. Lenders report two types of home equity loans to HMDA, homeimprovement loans and piggyback loans. ${ }^{5}$ We exclude all home improvement loans. As

\footnotetext{
${ }^{3}$ Feins, et al, pages $42-45$, provide descriptions of various lending arrangements. In short, the wholesale channel is when a broker acts as an intermediary between the borrower and the lender. There generally are two types of wholesale loans. The first are brokered loans, in which the loan is either table funded (closed in the lender's name) or concurrently funded (closed in the broker's name). The second are correspondent loans, in which a correspondent works in the name of a lender and a warehouse line of credit funds the loan. ${ }^{4}$ For a more detailed discussion of this issue, see Scheessele (1998).

${ }^{5}$ Some of the reasons people take out piggybacks are to meet a down payment requirement, to avoid paying mortgage insurance, or to reduce the size of their regular mortgage so that it falls within the conforming threshold.
} 
mentioned in the main paper, the 2004 HMDA data indicate whether a loan is a first lien or a second lien. We refer to home purchase or refinance loans that are second liens as "piggyback loans." Before 2004, we filtered out piggybacks from the HMDA data by excluding all home purchase and refinance loans for less than $\$ 25,000$. In 2004, we removed all piggyback loans. Lien status is available in HMDA for originated loans and loans that were sold, but not for purchased loans. For now, we assumed that a purchased loan was a junior lien if the loan amount was less than the mean size of originated loans. In the future, we plan to reexamine this assumption.

We compiled HMDA originations, purchases, and sales for each of the five types of originators in our mortgage system. So that the HMDA mortgage flows would be consistent with the mortgage debt outstanding from call reports (described in the next section), we included for each type of lender the mortgage flows of direct subsidiaries. For example, the mortgage flows for commercial banks include data from all HMDA reporters that either have a bank charter or are a direct subsidiary of a commercial bank.

\section{Regular Mortgage Debt Outstanding at HMDA Reporters ${ }^{6}$}

We compiled regular mortgage debt outstanding at HMDA reporters from financial reports filed by banks and thrifts with their regulators. Included are all federally insured lenders that have a commercial bank or savings association charter (including savings banks, savings and loans, cooperative banks, and industrial banks), and are headquartered in the U.S. Regular mortgage debt excludes all home equity loans (closed-end loans and home equity lines of credit) and construction loans. The reporting status of each HMDA respondent at the end of the year determined its classification for the entire year. For example, if an institution changed its charter from a thrift to a commercial bank in May, we would classify it as a commercial bank for the entire year, even though it actually was a thrift (and filed a TFR) at the end of the first quarter.

Through the end of 2003, the mortgage debt outstanding in the financial reports filed by thrifts ("Thrift Financial Reports" or TFRs, discussed in appendix B) did not distinguish between regular mortgages and home equity loans. We estimate RMDO at thrifts through the end of 2003 using the following method: First, we calculate the ratio of RMDO to total mortgage debt outstanding (excluding construction loans) at savings banks (for which we can distinguish between regular mortgages and home equity loans). Then, we multiply this ratio by MDO (excluding construction loans) at thrifts that report to both OTS and HMDA. ${ }^{7}$

\section{Adjustments to HMDA Sales}

For loans that were sold, HMDA records only the date on which the loan was acquired, not the date of sale. Because the reconciliation of the change in RMDO with the mortgage flows requires a measure of sales at the time of sale, we need to map HMDA sales from the time of acquisition to the time of sale. In addition, HMDA does not record

\footnotetext{
${ }^{6}$ Robert Avery at the Federal Reserve Board developed the method described in this section.

${ }^{7}$ This is the same method used by FOF.
} 
loans sold in the current year that were acquired in a previous year. We refer to this as "truncation bias." We use the following methods to estimate corrections for these problems.

\section{Truncation Bias}

The upper panel of table A-1 shows the average ratios of HMDA sales to acquisitions (originations plus purchases) for each quarter of the year from 1993 to 2004. For each type of originator, the table shows three ratios: total sales to total acquisitions, sales of originated loans to originations, and sales of purchased loans to purchases. In almost every case, the largest ratio occurs in the first quarter, the ratio declines slightly or is essentially flat in the second and third quarters, and then drops sharply in the fourth quarter. For example, at commercial banks (line 4) the ratio of total sales to total acquisitions averages 66 percent in the first quarter, is slightly lower in the second and third quarters, and drops to 48 percent in the fourth quarter. The only significant exception is the ratio for sales of purchased loans at savings banks (line 9), in which the share increases between the first and second quarters.

We use the following multiplicative adjustment factors to correct for truncation bias in HMDA:

$$
\operatorname{Adj}\{Q i\}=S\{Q 1\} / S\{Q i\}, i=1,2,3,4,
$$

where $\mathrm{S}\{\mathrm{Qi}\}$ is the ratio of sales to acquisitions and $\operatorname{Adj}\{\mathrm{i})$ is the adjustment factor for the ith quarter. The adjustment factors are shown in the lower panel of the table. Note that the method implies that the first-quarter adjustment factors equal one. The method assumes that no loans are sold more than a year after they are acquired and that the propensity to sell mortgages does not vary either from year-to-year or over the course of the year.

Of course, some sales occur more than a year after they are acquired, which implies that each of the adjustment factors contains at least a slight downward bias. In the future, we hope to incorporate data on sales of seasoned loans to the GSEs, which ought to correct a good part of this problem. In addition, we are looking into the feasibility of estimating time-varying adjustment factors that reflect, for example, how the propensity of originators to sell loans varies with the ARM share of originations.

For banks, thrift institutions, and mortgage companies, we separately adjusted the raw HMDA data on sales of originated and purchased loans. At savings banks, we adjusted total raw HMDA sales using the adjustment factor for total acquisitions (line 22).

\section{Timing-of-Sale Adjustment}

We employ two regressions in order to estimate the typical mapping from the quarter of acquisition to the quarter of sale. In the first equation, purchases reported by the GSEs and Ginnie Mae were regressed on current and lagged HMDA sales to those agencies. The second equation relates loan sales reported by thrifts that report to OTS (which 
record sales in the quarter the loan was sold) to current and lagged HMDA sales by thrifts regulated by OTS that also filed a HDMA report. ${ }^{8}$ The table below summarizes the results. The period of estimation was from 1993:Q1 to 2004:Q4. Note that in each regression, the sum of the coefficients exceeds one because HMDA coverage is less than 100 percent. $^{9}$

\begin{tabular}{rrrrrr}
\hline $\begin{array}{c}\text { Corrected } \\
\text { R-squared }\end{array}$ & Constant & Cales $(\beta 1)$ & Sales $(\beta 2)$ & $\begin{array}{c}\text { B1/ } \\
(\beta 1+\beta 2)\end{array}$ & $\begin{array}{c}\beta 2 / \\
(\beta 1+\beta 2)\end{array}$ \\
\hline $\begin{array}{c}\text { GSE Equation } \\
0.9877\end{array}$ & 10.7 & 0.7591 & 0.3045 & 0.7137 & 0.2863 \\
& $(2.7)$ & $(22.9)$ & $(9.2)$ & & \\
Equation for Thrifts Regulated by OTS \\
0.9823
\end{tabular}

The second column from the right shows the estimated proportion of loans acquired in the current quarter that were sold in the same quarter. The last column shows the proportion of loans acquired in the current quarter that were sold in the next quarter. Of loans purchased by the GSEs, the seller acquired about 71 percent in the same quarter, with the remaining 29 percent acquired one-quarter earlier. The results are almost the same at OTS reporters. The factors we used to correct for timing-of-sales bias were in between the two set of estimates. As shown in figures A-1 to A-4, the ratios of sales to acquisitions based on adjusted sales is much smoother than the ratios based on raw HMDA sales.

\section{E. The Share of Home Purchase Loans in HMDA Not for Owner-Occupancy} Loan applicants indicate whether a home purchase loan is for owner-occupancy or for some other purpose, such as to purchase a rental unit or a vacation home. As shown in figure A-5, the share of the number of home purchase loans in HMDA not for owneroccupancy has trended up steadily since the mid-1990s, averaging more than 15 percent in 2004.

For two reasons the level of the non-owner-occupied share of home purchase loans in HMDA probably is lower than the non-owner-occupied share of all homes purchased. First, because the terms of loans to purchase owner-occupied homes tend to be better than those for rental or vacation homes, some borrowers might indicate in the loan application that the home is for owner-occupancy when, in fact, it is intended to be a rental or vacation home. Second, according to the 2000 Residential Finance Survey

\footnotetext{
${ }^{8}$ In both regressions, we used HMDA sales adjusted for truncation bias.

${ }^{9}$ Another reason why the sum of the coefficients exceeded one is that HMDA excludes sales of seasoned loans.
} 
(RFS), the mortgaged share of owner-occupied purchases from 1999:Q1 to 2001:Q1 was about 87 percent, whereas the mortgaged share of other types of home purchases (including rental and vacation homes) was about 71 percent. 
A-1

Commercial Banks That Report Under HMDA

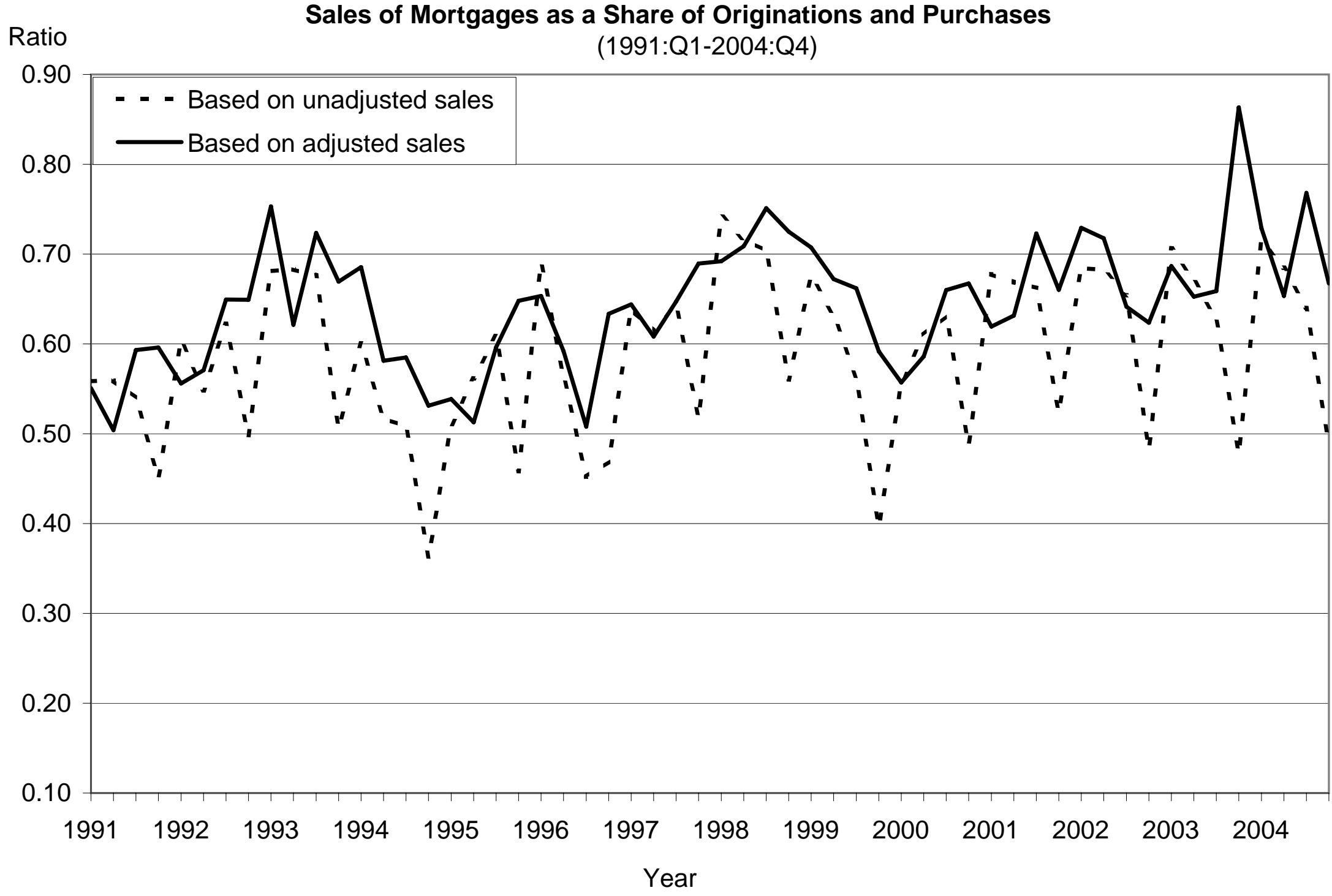


A-2

Thrift Institutions Regulated by the OTS That Report Under HMDA

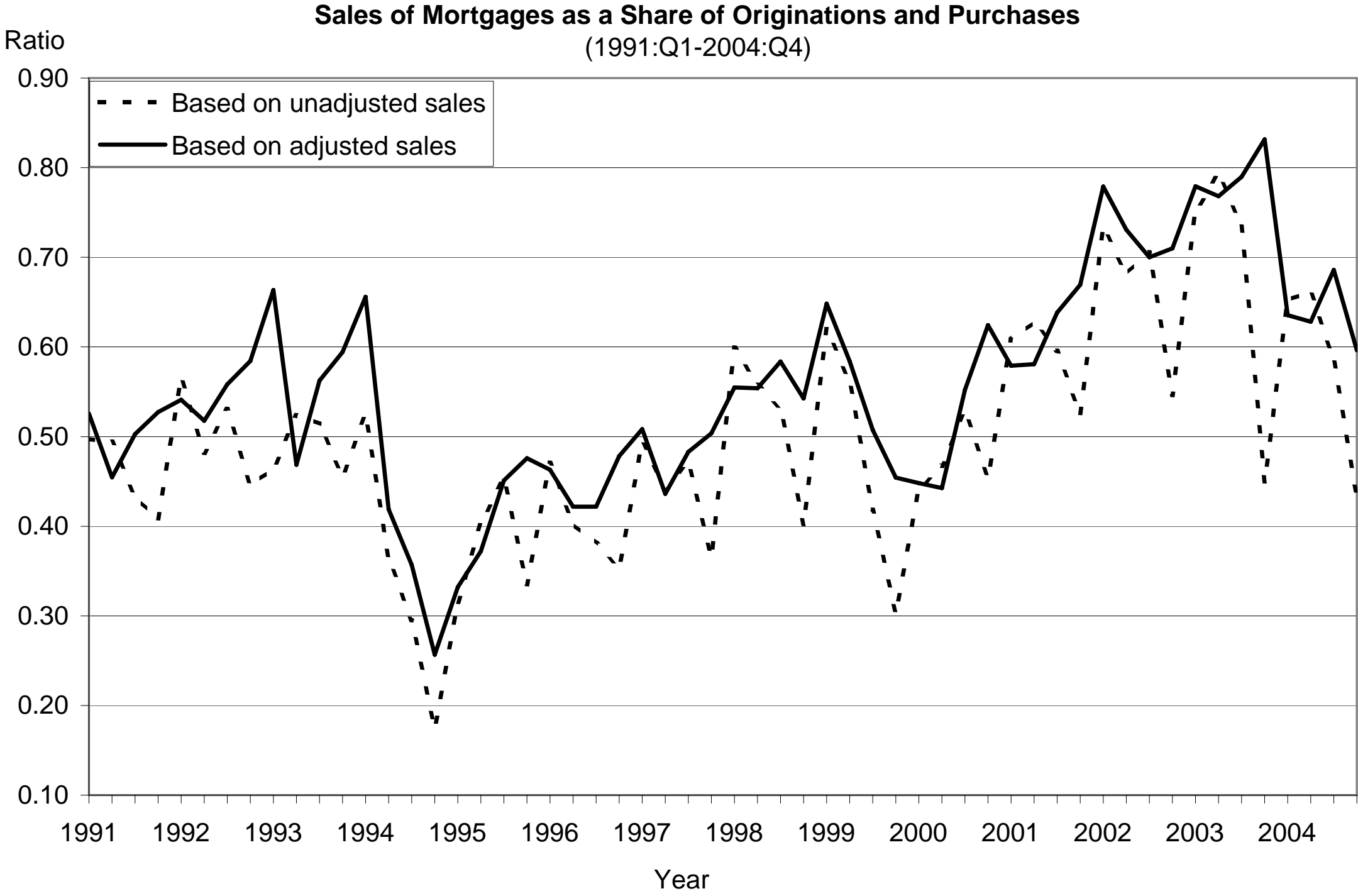


A-3

Savings Banks That Report Under HMDA

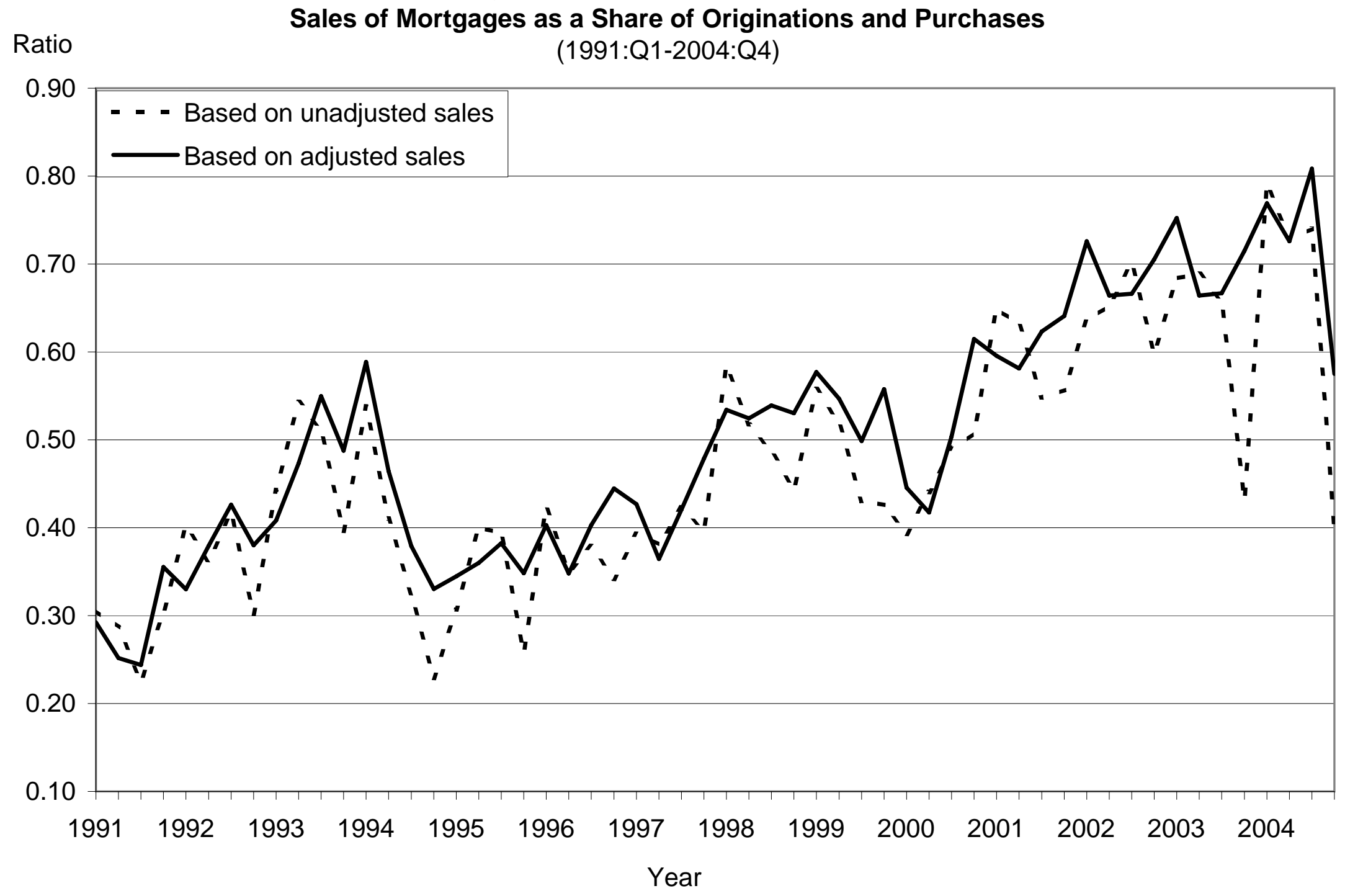


A-4

Mortgage Companies That Report Under HMDA

Sales of Mortgages as a Share of Originations and Purchases

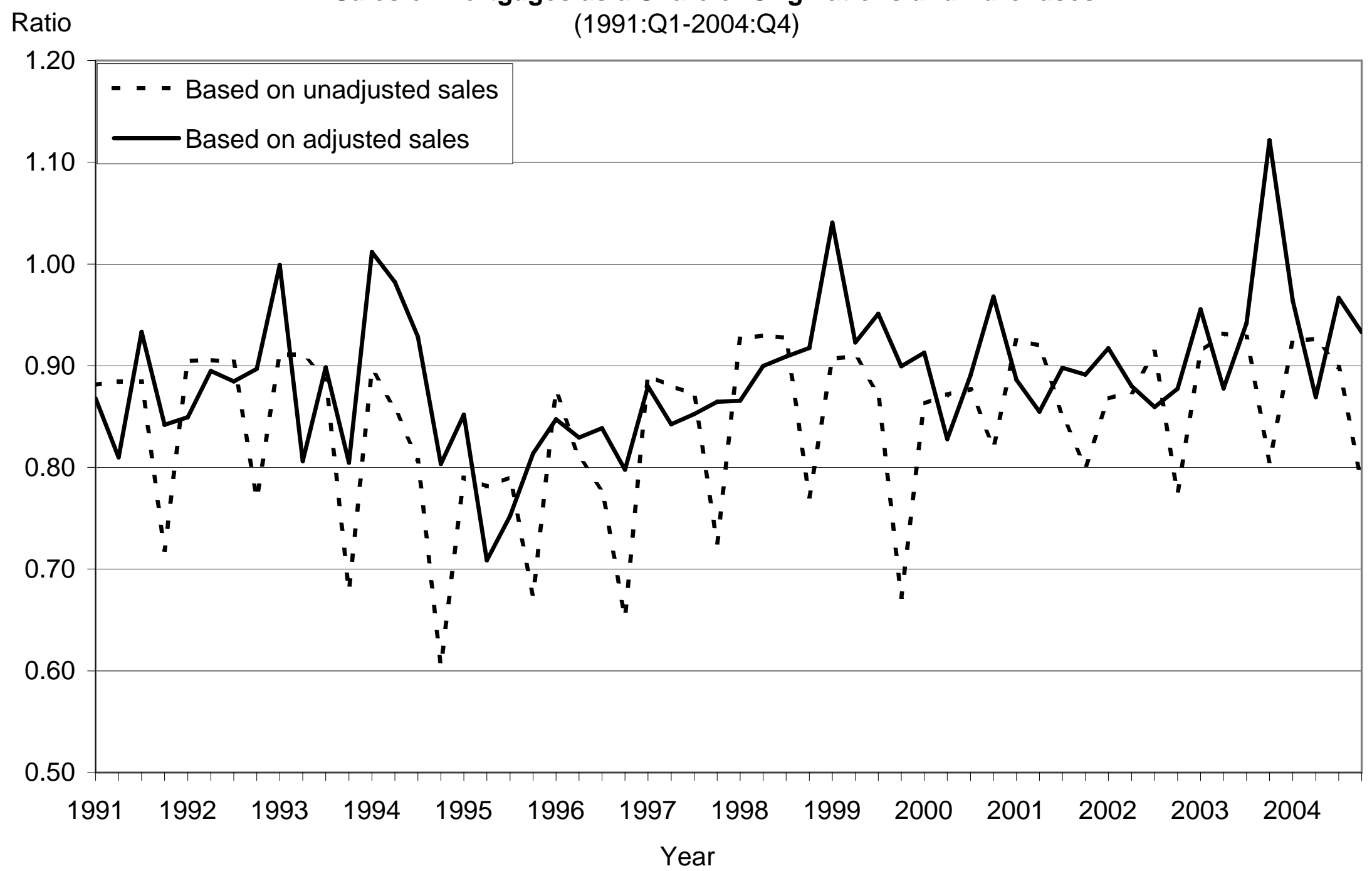


A-5

The Share of Home Purchase Loans in HMDA Not For Owner-Occupancy

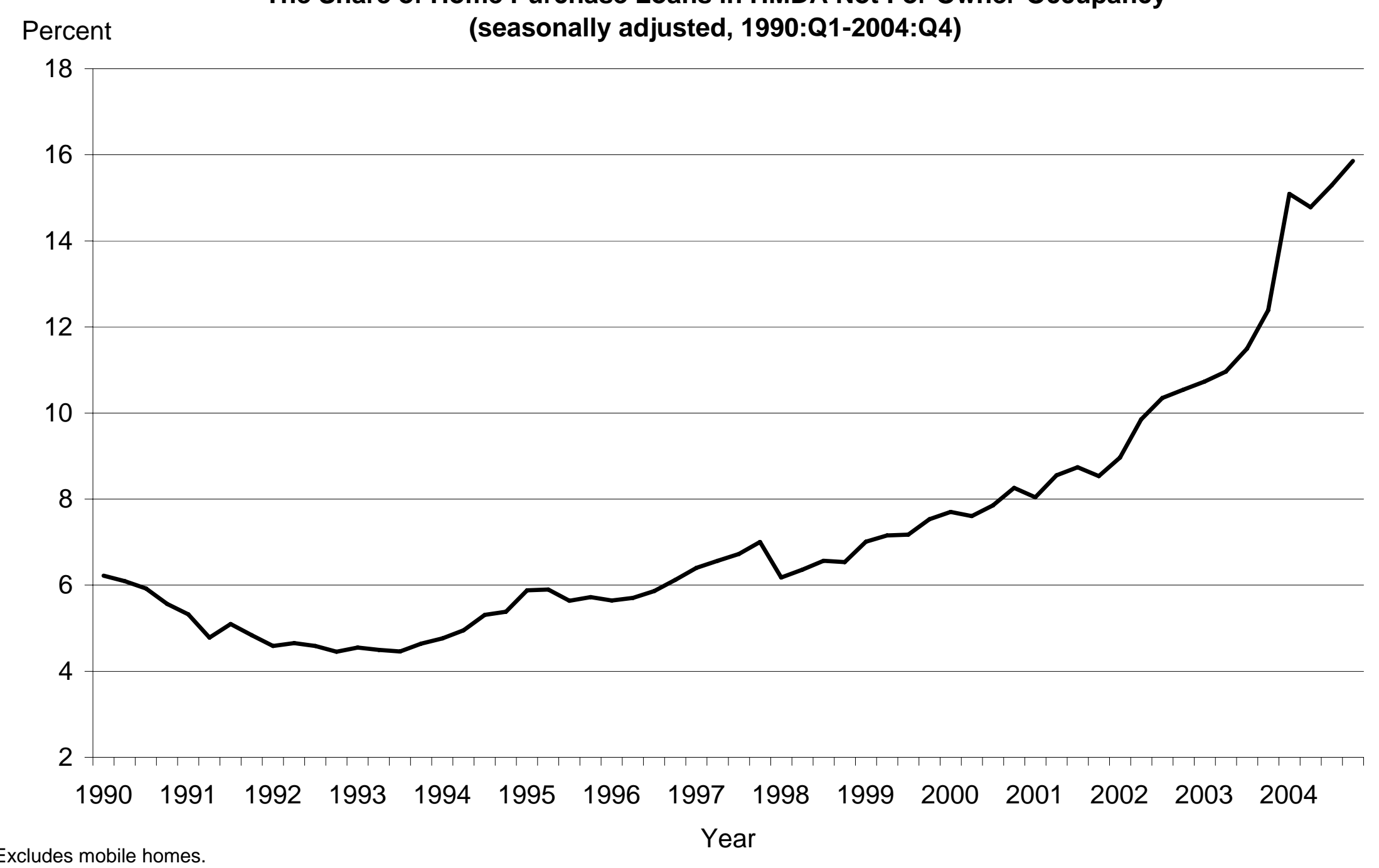

Excludes mobile homes. 
Table A-1

Ratio of Mortgage Sales to Acquisitions in HMDA and Implied Multiplicative Adjustment Factors (averages from 1993:Q1 to 2004:Q4)

\begin{tabular}{|c|c|c|c|c|c|}
\hline & & & Qua & & \\
\hline & & 1 & 2 & 3 & 4 \\
\hline & Ratio of Sales to Acc & & & & \\
\hline & All Originators & & & & \\
\hline (1) & Total acquisitions & 0.7085 & 0.6901 & 0.6795 & 0.5525 \\
\hline (2) & Originated loans & 0.7115 & 0.6904 & 0.6813 & 0.5582 \\
\hline (3) & Purchased loans & 0.7006 & 0.6896 & 0.6751 & 0.5338 \\
\hline & Commercial Banks & & & & \\
\hline (4) & Total acquisitions & 0.6564 & 0.6338 & 0.6142 & 0.4780 \\
\hline (5) & Originated loans & 0.6533 & 0.6333 & 0.6205 & 0.4826 \\
\hline (6) & Purchased loans & 0.6595 & 0.6312 & 0.6067 & 0.4685 \\
\hline & $\underline{\text { Savings Banks }}$ & & & & \\
\hline (7) & Total acquisitions & 0.5332 & 0.5221 & 0.5070 & 0.4154 \\
\hline (8) & Originated loans & 0.5451 & 0.5244 & 0.5116 & 0.4179 \\
\hline (9) & Purchased loans & 0.4475 & 0.4949 & 0.4422 & 0.3838 \\
\hline & OTS Reporters & & & & \\
\hline (10) & Total acquisitions & 0.5560 & 0.5405 & 0.5179 & 0.4000 \\
\hline (11) & Originated loans & 0.5722 & 0.5525 & 0.5250 & 0.4059 \\
\hline (12) & Purchased loans & 0.4722 & 0.4644 & 0.4475 & 0.3424 \\
\hline & Mortgage Companies & & & & \\
\hline (13) & Total acquisitions & 0.8906 & 0.8835 & 0.8669 & 0.7307 \\
\hline (14) & Originated loans & 0.9004 & 0.8903 & 0.8718 & 0.7452 \\
\hline (15) & Purchased loans & 0.8562 & 0.8571 & 0.8445 & 0.6781 \\
\hline & Implied Adjustment & & & & \\
\hline & All Originators & & & & \\
\hline (16) & Total acquisitions & 1.0000 & 1.0267 & 1.0427 & 1.2824 \\
\hline (17) & Originated loans & 1.0000 & 1.0305 & 1.0443 & 1.2747 \\
\hline (18) & Purchased loans & 1.0000 & 1.0160 & 1.0378 & 1.3125 \\
\hline & Commercial Banks & & & & \\
\hline (19) & Total acquisitions & 1.0000 & 1.0356 & 1.0687 & 1.3733 \\
\hline (20) & Originated loans & 1.0000 & 1.0315 & 1.0529 & 1.3537 \\
\hline (21) & Purchased loans & 1.0000 & 1.0448 & 1.0871 & 1.4078 \\
\hline & $\underline{\text { Savings Banks }}$ & & & & \\
\hline (22) & Total acquisitions & 1.0000 & 1.0212 & 1.0516 & 1.2835 \\
\hline (23) & Originated loans & 1.0000 & 1.0395 & 1.0655 & 1.3044 \\
\hline (24) & Purchased loans & 1.0000 & 0.9043 & 1.0120 & 1.1661 \\
\hline & OTS Reporters & & & & \\
\hline (25) & Total acquisitions & 1.0000 & 1.0286 & 1.0734 & 1.3898 \\
\hline (26) & Originated loans & 1.0000 & 1.0357 & 1.0900 & 1.4099 \\
\hline (27) & Purchased loans & 1.0000 & 1.0168 & 1.0554 & 1.3793 \\
\hline & Mortgage Companies & & & & \\
\hline$(28)^{7}$ & Total acquisitions & 1.0000 & 1.0080 & 1.0274 & 1.2188 \\
\hline (29) & Originated loans & 1.0000 & 1.0113 & 1.0328 & 1.2082 \\
\hline (30) & Purchased loans & 1.0000 & 0.9989 & 1.0138 & 1.2625 \\
\hline
\end{tabular}




\section{Appendix B \\ The Estimation Repayments and Originations}

\section{A. Overview}

For each of the eleven mortgagees in our system, we solve the following identity:

$$
\Delta \mathrm{M}_{\mathrm{i}} \equiv \mathrm{O}_{\mathrm{i}}+\mathrm{P}_{\mathrm{i}}-\mathrm{S}_{\mathrm{i}}-\mathrm{R}_{\mathrm{i}}+\mathrm{C}_{\mathrm{i}}
$$

$\Delta \mathrm{M}_{\mathrm{i}}$ is the change in RMDO from the Federal Reserve Board's flow of funds accounts at the ith type of mortgagee. $\mathrm{O}_{\mathrm{i}}, \mathrm{P}_{\mathrm{i}}, \mathrm{S}_{\mathrm{i}}$, and $\mathrm{R}_{\mathrm{i}}$ denote originations, purchases, sales, and repayments (or liquidations) of mortgages, respectively. $\mathrm{C}_{\mathrm{i}}$ denotes the effects on RMDO at the $i$ th type of originator of mergers, charter changes, and acquisitions of direct subsidiaries (M\&A). ${ }^{1}$ All of the variables in (1) pertain only to regular mortgages. Time subscripts are omitted from (1) for convenience.

We estimate (1) for five types of originators: commercial banks; thrift institutions regulated by the Office of Thrift Supervision (OTS); thrift institutions regulated by the Federal Deposit Insurance Corporation, which we refer to as savings banks; credit unions; and mortgage companies (hereafter, we refer to the first four types collectively as depository institutions). We also estimate (1) for six categories of non-originators: three government-sponsored enterprises (Fannie Mae, Freddie Mac, and the Federal Home Loan Banks); Ginnie Mae; issuers of private mortgage-backed securities; and all other types of mortgagees, including households, government agencies, pension funds, and life insurance companies.

We assume, as does the flow of funds accounts, that home mortgage transactions with the rest of the world are zero; hence, total purchases equals total sales, and the effects of M\&A on RMDO sum to zero. ${ }^{2}$ Thus, summing across the eleven types of mortgagees and rearranging terms yields originations:

$$
\mathrm{O}=\Delta \mathrm{M}+\mathrm{R}
$$

\section{B. Originations and Repayments at Depository Institutions \\ Bank and Thrift Institutions}

To solve (1) for bank and thrift institutions, we use FOF's change in RMDO, estimate originations, purchases, sales, and repayments from HMDA, and solve for the effects of M\&A at HMDA reporters as a residual. We map from HMDA to the universe in each sector based on the ratio of RMDO at all lenders to RMDO at HMDA reporters (denoted $\mathrm{m}_{\mathrm{i}}$ ) as follows:

$$
\mathrm{m}_{\mathrm{i}} \equiv\left(\mathrm{M}_{\mathrm{i}, \mathrm{t}-\mathrm{l}} / \mathrm{MH}_{\mathrm{i}, \mathrm{t}, \mathrm{b}}\right)
$$

\footnotetext{
${ }^{1}$ The financial reports submitted by bank and thrift institutions to their primary regulators include the assets of direct subsidiaries.

${ }^{2}$ The amount of mortgage debt on U.S. homes that is held abroad is deemed negligible, but significant quantities of mortgage-backed securities are held abroad.
} 
$\mathrm{M}_{\mathrm{i}, \mathrm{t}-1}$ denotes RMDO from the FOF accounts at the $i$ th originator at the end of $\mathrm{t}-1$, and $\mathrm{MH}_{\mathrm{i}, \mathrm{t}, \mathrm{b}}$ denotes RMDO at HMDA reporters at the beginning of $\mathrm{t}^{3}{ }^{3}$ The mortgage assets of HMDA reporters are from the financial reports they file with their federal regulators. Total originations in the $i$ th sector equals HMDA originations multiplied by $\mathrm{m}_{\mathrm{i}}$ :

$$
\mathrm{O}_{\mathrm{i}} \equiv \mathrm{m}_{\mathrm{i}} * \mathrm{OH}_{\mathrm{i}}
$$

where $\mathrm{OH}$ denotes originations at HMDA reporters (figure B-1 shows total originations at commercial banks and thrifts that report to OTS). We assume the same mapping applies to purchases and sales:

$$
\begin{aligned}
& \mathrm{P}_{\mathrm{i}} \equiv \mathrm{m}_{\mathrm{i}} * \mathrm{PH}_{\mathrm{i}} \\
& \mathrm{S}_{\mathrm{i}} \equiv \mathrm{m}_{\mathrm{i}} * \mathrm{SH}_{\mathrm{i}}
\end{aligned}
$$

where $\mathrm{PH}$ and $\mathrm{SH}$ denote purchases and sales at HMDA reporters. The variable $\mathrm{SH}$ in (6) is adjusted HMDA sales, described in appendix A.

We estimate total repayments in each sector by first estimating repayments at HMDA reporters and then extrapolating to the universe. The following identity defines repayments at HDMA reporters $(\mathrm{RH})$ :

$$
\mathrm{RH}_{\mathrm{i}} \equiv \mathrm{OH}_{\mathrm{i}}+\mathrm{PH}_{\mathrm{i}}-\mathrm{SH}_{\mathrm{i}}-\Delta \mathrm{MHx}_{\mathrm{i}}
$$

$\Delta \mathrm{MHx}_{\mathrm{i}}$ is the change in regular RMDO at HMDA filers resulting from acquisitions net of sales and repayments; by design, it excludes the effects on RMDO of M\&A and of changes in the institutions that report under HDMA. ${ }^{4}$ The implied repayment rate at HMDA filers $\left(r_{i}\right)$ is

$$
\mathrm{r}_{\mathrm{i}}=\mathrm{RH}_{\mathrm{i}} / \mathrm{MH}_{\mathrm{i}, \mathrm{t}, \mathrm{b}}
$$

We assume that the repayment rates are the same at HMDA and non-HMDA reporters. ${ }^{5}$ Hence, total repayments at the $i$ th originator is by definition the repayment rate at HMDA reporters multiplied by RMDO at the end of the previous period:

\footnotetext{
${ }^{3}$ See appendix A for further details.

${ }^{4}$ By definition, $\triangle \mathrm{MHx}_{\mathrm{i}}$ equals to the change in RMDO at HMDA reporters from the beginning to the end of the current quarter. $\triangle \mathrm{MHx}$ excludes the effects on RMDO resulting from changes in the institutions that report under HMDA and M\&A. As discussed in appendix A, we incorporate at the beginning of the year changes in the lenders that report under HMDA and all M\&A that occur during that year; consequently, $\mathrm{MH}_{\mathrm{i}, \mathrm{t}, \mathrm{b}}$ differs from $\mathrm{MH}_{\mathrm{i}, \mathrm{t}-\mathrm{l}, \mathrm{e}}$ only in the first quarter.

${ }^{5}$ At savings banks, the estimated repayment rates were negative in 1990:Q3 and in the first quarters of 1991, 1992, and 1993. Robert Avery, who compiled the data on RMDO at savings banks that report under HMDA, noted that although the FFIEC variable definitions have not changed since 1990 “...it appears that at least informal treatment of several items changed in some of the earlier calendar years. This is a particular problem with thrifts filing FFIEC reports. There are unexplainable significant increases in... mortgage[s] outstanding....at the beginning of 1992 and 1993 that cannot be explained by mergers or changes in which report (TFR or FFIEC) is filed. Discussions with contacts at the FDIC have not revealed an explanation." (From an internal Federal Reserve Board memo to Chairman Greenspan in July 2002)
} 


$$
\mathrm{R}_{\mathrm{i}} \equiv \mathrm{r}_{\mathrm{i}} * \mathrm{M}_{\mathrm{i}, \mathrm{t}-1}
$$

We then estimate the effects of mergers, acquisitions of direct subsidiaries, and charter changes on RMDO as a residual:

$$
\mathrm{C}_{\mathrm{i}}=\Delta \mathrm{M}_{\mathrm{i}}-\left(\mathrm{O}_{\mathrm{i}}+\mathrm{P}_{\mathrm{i}}-\mathrm{S}_{\mathrm{i}}-\mathrm{R}_{\mathrm{i}}\right)
$$

That is, mergers, acquisitions, and charter changes are defined as the part of the change in RMDO that is not explained by acquisitions net of sales and repayments.

\section{Credit Unions}

The method for credit unions is similar to that used for banks and thrift institutions. The main difference is that we use data from regulatory financial statements that credit unions file with the National Credit Union Association (NCUA) in place of data reported under HMDA. The NCUA data are described in section F of this appendix.

\section{Repayments at Mortgagees that Do Not Originate Loans}

For each of the mortgagees that do not originate loans, we back out repayments by rearranging the terms in (2) and setting originations and M\&A equal to zero:

$$
R_{i} \equiv P_{i}-S_{i}-\Delta M_{i}
$$

The GSEs, Ginnie Mae, and Private MBS

For Freddie Mac and Fannie Mae, the source data on purchases and RMDO (both their retained portfolios and securitized pools) are from their own reports or those of their regulator, the Office of Federal Housing Enterprise Oversight (OFHEO). The data for the Federal Home Loan Banks are from the Federal Housing Finance Board and the data for Ginnie Mae are from that agency. For private mortgage-backed securities, RMDO is from FOF, and purchases (issuance) are from Inside Mortgage Finance's publication "Inside ABS and MBS."

For the GSEs (Fannie Mae, Freddie Mac, and the Federal Home Loan Banks) and Ginnie Mae, RMDO is from the Flow of Funds accounts. FOF constructs RMDO for the GSEs from reports filed by those agencies that distinguish between 1-4 and multifamily mortgage debt. Purchases and sales are from reports filed by the agencies; we back out repayments as a residual.

The vast majority of loans purchased by the GSEs and Ginnie Mae are regular mortgages. We had to make some assumptions to estimate sales of 1-4 mortgages and to filter out

\footnotetext{
We inserted projected values of the repayment rate in the quarters with negative values. The projected values are from equations that relate the savings bank repayment rate to the GSE repayment rate. In order to balance the identity that relates the change in RMDO at HMDA reporters to HMDA's mortgage flows, we adjusted the change in RMDO at HMDA reporters to deliver the level of implied repayments consistent with the projected repayment rate. We used the same method to correct a negative repayment rate at thrifts regulated by OTS in the first quarters of 1993 and 2004.
} 
multifamily loans from total purchases. To estimate purchases and sales of regular mortgages by Fannie Mae and Freddie Mac, we used data from annual reports published by OFHEO and quarterly and monthly statements issued by the companies.

\section{Other Mortgagees That Do Not Originate Loans}

RMDO for the "other mortgagees" group is a residual equal to total RMDO minus RMDO at commercial banks, thrift institutions, credit unions, mortgage companies, the on-balance-sheet holdings of the GSEs, pools securitized by the GSEs, loans securitized by Ginnie Mae, and private MBS. ${ }^{6}$ The other mortgagees group has accounted for about 5 percent of RMDO in recent years.

\begin{tabular}{lc} 
& $\begin{array}{c}\text { Composition of Regular Mortgage } \\
\text { Debt Outstanding at Other Mortgagees } \\
\text { (percent, 2004:Q4) }\end{array}$ \\
\hline Other mortgagees & 100.0 \\
Households & 34.7 \\
Nonfinancial corporate businesses & 7.6 \\
Nonfarm noncorporate businesses & 3.8 \\
State and local governments & 19.1 \\
Federal government & 4.2 \\
Bank personal trusts and estates & 0.6 \\
Life insurance companies & 1.3 \\
Private pension funds & 0.4 \\
State and local government retirement funds & 1.9 \\
REITS & 25.6 \\
Unspecified GSEs &
\end{tabular}

Some of the members of the other mortgagees group originate loans. However, because the originations of the mortgagees in this group are a tiny fraction of the total, and because for most of the members of this group it is difficult to find time series data on originations, we decided to assume for now that originations in this category are zero. We assume that the repayment rate at other mortgagees equals the average elsewhere in our mortgage system (denoted $r$ ). These assumptions allow us to back out net purchases as:

$$
\left(P_{i}-S_{i}\right)=\Delta M_{i}+r * M_{i, t-1}
$$

\section{Originations and Repayments at Mortgage Companies}

Because mortgage companies do not file regulatory financial reports, we cannot calculate the RMDO at HMDA reporters in this sector and thus cannot map directly from HMDA's mortgage flows to the universe as in (5) to (7). As an alternative, we estimate originations at mortgage companies as a residual by rearranging the terms in (1):

$$
\mathrm{O}_{\mathrm{i}} \equiv \Delta \mathrm{M}_{\mathrm{i}, \mathrm{t}}+\left(\mathrm{S}_{\mathrm{i}}-\mathrm{P}_{\mathrm{i}}\right)+\left(\mathrm{r}_{\mathrm{i}} * \mathrm{M}_{\mathrm{i}, \mathrm{t}-1}\right)-\mathrm{C}_{\mathrm{i}}
$$

${ }^{6} \mathrm{FOF}$ assumes that finance companies hold only home equity loans.

${ }^{7}$ Primarily Farmers Home Administration pools. 
We solve this identity with the following data:

- The change in RMDO is from FOF.

- As mentioned above, we assume that, in the aggregate, total sales equals total purchases, which implies that net sales at mortgage companies equals net purchases at all other types of mortgagees (see lines 325 to 330 of table 1).

- We assume that the repayment rate at mortgage companies $\left(r_{i}\right.$ in the equation above) equals the average at depository institutions.

- Overall, mergers and acquisitions net to zero. Thus, negative one multiplied by the sum of M\&A at depository institutions equals M\&A at mortgage companies (see lines 331 to 334 and 341 of table 1).

As shown in lines 335-341 of table 1, net sales are by far the dominant component of mortgage company originations. During the 1991-2004 period, net sales averaged about $\$ 620$ billion per year. By contrast, during the same period, the change in RMDO averaged negative $\$-1-1 / 2$ billion per year, repayments averaged less than $\$ 10$ billion, and $M \& A$ averaged close to zero.

\section{E. A Note on Data from Thrift Financial Reports (TFR)}

The TFRs request information on "loan disbursements" rather than gross originations. Disbursements are the same as gross originations for home-purchase loans and for refinance loans in which the thrift did not hold the loan retired at the time of the refi. However, if a thrift originates a refinance loan and also held in portfolio the retired loan (an "own refi"), the loan disbursement is the net increase in the loan balance. For this reason, we do not employ directly the TFR data in our mortgage system.

\section{F. A Note on Data from the National Credit Union Association (NCUA)}

The National Credit Union Association (NCUA) 5300 report shows data on mortgage originations, purchases, sales, and RMDO. It distinguishes between regular mortgages and home equity loans for originations, sales, and mortgage debt outstanding. The NCUA report does not break out first liens for purchases, but that shortcoming is inconsequential because credit unions do not purchase many residential mortgages.

The NCUA data begin in 1990; however, only annual data are available in that year. We filled in quarterly values for originations and sales by interpolating the annual value. ${ }^{8}$ In 1991, NCUA data are available only for the first and second halves of the year. Then, from the beginning of 1992 to 2002:Q2, the NCUA data are available quarterly; however, the number of institutions reporting falls off sharply in the first and third quarters, rendering those data useless for our purposes. We dealt with this problem by calculating repayment rates for half-year intervals from 1991 to the middle of 2002, and applied a simple interpolation in order to create quarterly rates. Since the middle of 2002, the

\footnotetext{
${ }^{8}$ Because mortgage debt outstanding at NCUA reporters is unavailable before the fourth quarter of 1990 , we cannot calculate repayment rates in that year. As a substitute, we estimated the quarterly repayment rates at credit unions based on the repayment rates at OTS reporters.
} 
NCUA has required all reporting institutions to file quarterly reports, allowing us to calculate quarterly repayment rates beginning in the middle of that year.

The NCUA did not report loan purchases until 1996. Before then, we estimated the level of purchases as the level of sales multiplied by the average ratio of purchases to sales during the 1996 to 2001 period. FOF's RMDO for credit unions is about 11 percent larger than NCUA RMDO, mainly because credit unions in Ohio do not report to the NCUA, but they are included in the FOF series.

We assume that credit unions do not engage in any M\&A outside of their sector, and solve for originations as the change in RMDO plus net sales plus repayments. The change in RMDO is from FOF. Sales and purchases equal the NCUA series multiplied by the ratio total RMDO at credit unions (FOF) to RMDO at NCUA reporters.

Repayments equal the repayment rate at NCUA reporters multiplied by FOF's RMDO at the end of the previous quarter (lines 217 to 228 of table 1).

\section{G. Mergers, Acquisitions, and Charter Changes}

The repayment rate HMDA reporters equals:

$$
\mathrm{r}_{\mathrm{i}}=\mathrm{RH}_{\mathrm{i}} / \mathrm{MH}_{\mathrm{i}, \mathrm{t}, \mathrm{b}}=\left(\mathrm{OH}_{\mathrm{i}}+\mathrm{PH}_{\mathrm{i}}-\mathrm{SH}_{\mathrm{i}}-\Delta \mathrm{MHx}_{\mathrm{i}}\right) / \mathrm{MH}_{\mathrm{i}, \mathrm{t}, \mathrm{b}}
$$

where the subscript i denotes commercial banks, thrifts regulated by OTS, or savings banks. Total repayments are given by:

$$
\mathrm{R}_{\mathrm{i}}=\mathrm{r}_{\mathrm{i}} * \mathrm{M}_{\mathrm{i}, \mathrm{t}-1}
$$

Multiplying through by $\left(\mathrm{M}_{\mathrm{i}, \mathrm{t}-1} / \mathrm{M}_{\mathrm{i}, \mathrm{t}-1}\right)$ and combining terms yields:

$$
\mathrm{R}_{\mathrm{i}}=\mathrm{m}_{\mathrm{i}} * \mathrm{RH}_{\mathrm{i}}
$$

Thus, implied repayments at HMDA reporters map directly to total repayments via the factor $\mathrm{m}$.

The change in RMDO may be expressed as:

$$
\Delta \mathrm{M}_{\mathrm{i}} \equiv \mathrm{O}_{\mathrm{i}}-\mathrm{P}_{\mathrm{i}}+\mathrm{S}_{\mathrm{i}}-\left\{\left(\mathrm{OH}_{\mathrm{i}}+\mathrm{PH}_{\mathrm{i}}-\mathrm{SH}_{\mathrm{i}}-\Delta \mathrm{MHx}_{\mathrm{i}}\right) / \mathrm{MH}_{\mathrm{i}, \mathrm{t}, \mathrm{b}}\right\} \mathrm{M}_{\mathrm{i}, \mathrm{t}-1}-\mathrm{C}_{\mathrm{i}}
$$

Combining terms:

$$
\Delta \mathrm{M}_{\mathrm{i}}=\mathrm{m}_{\mathrm{i}} \Delta \mathrm{MHx}_{\mathrm{i}}-\mathrm{C}_{\mathrm{i}}
$$

Rearranging the terms in (19) defines mergers and acquisitions:

$$
\mathrm{C}_{\mathrm{i}}=\Delta \mathrm{M}_{\mathrm{t}}-\mathrm{m}_{\mathrm{i}} \Delta \mathrm{MHx}_{\mathrm{t}}
$$




\section{H. Further Discussion of Some Potential Sources of Error in Our Mortgage System}

This section discusses some of the potential sources of error in the mortgage system and their ramifications, especially to our estimates of originations.

The Mapping from HMDA to the Universe for Purchases

The mapping from HMDA purchases to the universe at banks and thrifts essentially assumes that the ratio of purchases at non-HMDA reporters to those at HMDA reporters equals the ratio of their respective RMDO. However, non-HMDA reporters - at least those that are relatively small— probably purchase fewer loans relative to their RMDO than HMDA reporters. If true, this would imply that our estimate of total purchases contains an upward bias. However, a bias to purchases would result in a bias to total originations only if it engendered a bias to either total RMDO or repayments. It turns out that the only way in which a bias to purchases would result in a bias total originations is if the repayment rate at HMDA reporters differs from that at non-HMDA reporters.

\section{The Adjustments to HMDA Sales}

All else equal, if the adjustments to HMDA sales to correct for truncation bias were too large in a given period, the repayment rate would be biased down, and, as a result, both repayments and total originations would be understated. Regarding the timing-of-sale adjustment, suppose that sales at time $t$ actually are greater than we assume, matched by an identical error in the opposite direction at $\mathrm{t}-1$. This would impart a downward bias to the repayment rate at $t$, and an upward bias to the rate at $t-1$; the effects of these biases on total originations would wash out across the two periods.

\section{Sales of Seasoned Loans}

The adjustments to HMDA sales do not account for sales of seasoned loans. All else equal, the exclusion of seasoned loans implies that net purchases at banks and thrift institutions are overstated, which would result in an upward bias to net sales and originations at mortgage companies, and thus to total originations. In the future, we hope to obtain data on seasoned loan purchases by the GSEs, and use those data to make an adjustment. 
Figure B-1

Billions of dollars

Regular Mortgage Originations

(annual, 1991 to 2004)

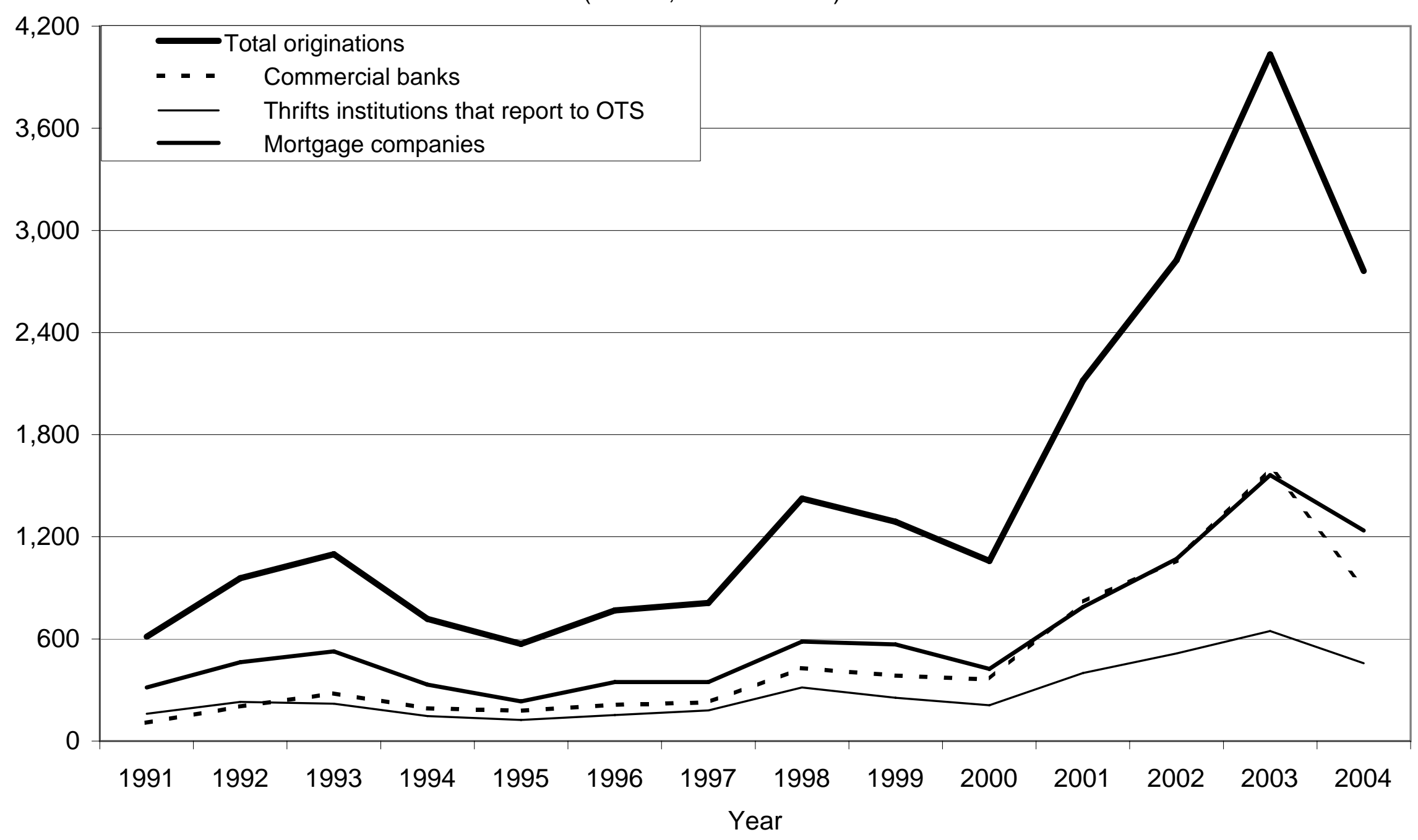

Note: credit unions and savings banks are not shown separately. 


\section{Appendix C \\ Data Sources for Private MBS}

Issuers of private (or non-agency) mortgage backed securities purchase loans and issue securities backed by those loans. ${ }^{1}$ Their purchases include jumbo mortgages, subprime regular mortgages, home equity loans, and or Alt-A loans (mortgages within the conforming threshold that do not meet the standards of Fannie Mae and Freddie Mac). We estimate repayments of private MBS as the value of non-agency issuance minus the change in RMDO from FOF. The repayment rate equals repayments divided by the level of RMDO at the end of the previous period.

Inside Mortgage Finance Publications Inc. (“Inside”) reports data on total non-agency MBS and two components, Alt-A loans and subprime loans. ${ }^{2}$ Inside's non-agency MBS series contains a significant break between 2000 and 2001: it excludes subprime regular mortgages before 2001, and includes home equity loans beginning in 2001. An analyst at Inside provided us with a decomposition of total non-agency MBS beginning in 1995 that corrects the break in the series and allows us to distinguish, for the most part, between regular mortgages and home equity loans. ${ }^{3}$ Line 299 of table 1 shows non-agency MBS excluding subprime and home equity loans. Lines 300 and 301 show private MBS issuance of subprime and home equity loans, respectively. Line 302 shows total private MBS issuance of regular mortgages, the sum of lines 299 and $300 .^{4}$

The calculated repayment rate on private MBS was negative in the second quarter of 1991. Accordingly, we estimated the repayment rate in that quarter based on the GSE repayment rate, and adjusted non-agency MBS such that it was consistent with the estimated repayment rate and FOF's change in RMDO.

\footnotetext{
${ }^{1}$ SPVs are “...similar to federally related mortgage pools in that they are not actual institutions, but are created for bookkeeping purposes.” Their assets “...have been transferred from the balance sheets of the institutions that originated the loans (in most cases commercial banks, thrift institutions, and finance companies) to the balance sheets of the SPVs.” (Guide to the Flow of Funds Accounts, Volume I, page 630). See Bruskin (2001) for a review of the nonagency MBS market.

2 The data are from “Inside MBS \& ABS, Electronic Edition.” Inside’s non-agency MBS series may exclude securities not registered with the SEC, which are a small share of the total. Alt-A loans generally have a FICO score that is in the range for conforming loans, but other problems make them unsuitable to Freddie and Fannie. Alt-A loans include some zero-down payment loans and jumbos.

3 A small percentage of the subprime loans shown in line 300 of table 1 actually are home equity loans.

4 Before 1995, total non-agency MBS excluding subprime loans is from Inside's annual publications. Subprime issuance in 1994 also is from the annual publication. The figures in table 1 for issuance of subprime non-agency MBS before 1994 and home equity issuance before 1995 are our estimates.
} 


\section{Appendix D \\ Mortgage Companies}

As mentioned in the body of the paper, FOF has held RMDO at mortgage companies constant since the end of 1997, when HUD's Survey of Mortgage Lending Activity (SMLA) was discontinued. However, the HMDA mortgage flows suggest that RMDO at mortgage companies has trended up since then. The first section of this appendix provides a brief description of the types of lenders included in FOF's mortgage company group. The next section presents evidence from HMDA that suggests RMDO at mortgage companies has risen since 1997. We then present simulated paths for RMDO at mortgage companies that are consistent with the HMDA.

\section{Definition of Mortgage Companies}

The "Guide to the Flow of Funds Accounts" provides the following description of mortgage brokers and bankers:

"Mortgage brokers originate mortgage loans, which they then sell to portfolio lenders...GSEs, and mortgage bankers...Mortgage bankers both originate mortgage loans and purchase them from brokers; they sell mortgages to portfolio lenders and GSEs, and they pool and securitize mortgages for sale as mortgagebacked securities. Both broker and bankers sell virtually all their mortgages in the secondary markets.” (Volume 1, p. 650)

The FOF mortgage company group includes lenders that do not have a bank, thrift, or credit union charter and also are not a direct subsidiary of a bank or thrift. Included are independent mortgage companies, those that are indirect subsidiaries of a bank or thrift institution, and mortgage companies that are members of a holding company. No comprehensive data are available on the mortgage assets held by any of these entities. For example, although some independent mortgage companies report to the SEC, the SEC does not ask them to break out 1-4 mortgage assets.

In HMDA, we identify all filers that are banks, thrifts, or credit unions and their direct subsidiaries. All of the remaining HMDA reporters are in the mortgage company group, which also includes mortgage banks, mortgage brokers, and some finance companies.

\section{The Change in RMDO Implied by HMDA's Mortgage Flows}

Through 1997, FOF based its estimate of RMDO at mortgage companies on information from SMLA; since then it has held RMDO flat at \$21.8 billion. A constant level of RMDO implies:

$$
\mathrm{O}+\mathrm{P}-\mathrm{S}=\mathrm{R}-\mathrm{C}
$$

where $\mathrm{O}, \mathrm{P}, \mathrm{S}, \mathrm{R}$, and $\mathrm{C}$ denote originations, purchases, sales, repayments, and mergers and acquisitions, respectively. During the 1998-2004 period, net acquisitions at mortgage companies that reported to HMDA (the left-hand side of (1)) averaged more than $\$ 70$ billion per year (line 4 of table D-1). On average, mortgage companies sold close to 92 
percent of the loans they acquired during that period (line 6), much higher than the typical shares at commercial banks and thrift institutions (about two-thirds). ${ }^{1}$

In order to gauge the consistency of FOF's RMDO with HMDA's mortgage flows we conducted a simulation beginning in 1998 that backs out implied repayments as:

$$
\mathrm{R}=\mathrm{O}+\mathrm{P}-\mathrm{S}-\Delta \mathrm{M}+\mathrm{C}
$$

Lines 1 to 3 of the table show HMDA originations, purchases, and adjusted sales. We do not know RMDO at HMDA reporters and therefore cannot calculate the blow-up factor $m$ to map from HMDA to the universe (described in appendix B). For the purposes of the simulation, we assume that HMDA's coverage of mortgage companies was 85 percent from 1998 to the present, and inflate net acquisitions accordingly (line 5). As mentioned above, RMDO (line 8) is constant over the simulation period. We calculate implied repayments from (2), ignoring M\&A for now. ${ }^{2}$

The implied repayment rate is equal to implied repayments divided by FOF's RMDO at the end of the previous year. As shown in line 10, the implied repayment rate averaged about 380 percent annually from 1998 to 2004, indicating clearly that something is amiss: either net acquisitions are too high or the change in RMDO is too low. The implied repayment rate averaged close to 190 percent from 1993 to 1997, also doubtless too high.

Note that it is impossible to generate a reasonable repayment rate by raising or lowering assumed HMDA coverage. For example, if we were to assume that HMDA coverage of mortgage companies was 100 percent, the simulated repayment rate from 1998 to 2004 would average close to 325 percent; alternatively, HMDA coverage of 60 percent would imply a repayment rate of more than 500 percent.

\section{Simulations of the Implied Change in RMDO at Mortgage Companies}

In the next two simulations, instead of using FOF's RMDO, we rearrange the terms in (2) and calculate the change in RMDO implied by net acquisitions from HMDA and repayments. In the simulation, repayments equal the average repayment rate at banks and thrift institutions (r) multiplied by the simulated level of RMDO at mortgage companies at the end of the previous quarter:

$$
\Delta \mathrm{M}_{\mathrm{t}}=\mathrm{O}_{\mathrm{t}}+\mathrm{P}_{\mathrm{t}}-\mathrm{S}_{\mathrm{t}}-\left(\mathrm{r} * \mathrm{M}_{\mathrm{t}-1}\right)
$$

The simulations run from the beginning of 1998 to the end of 2004 . The starting value for RMDO in 1997:Q4 is from FOF, \$21.8 billion.

The first simulation assumes that HMDA's coverage of mortgage company flows is 85 percent; that is, $\mathrm{OH} / \mathrm{O}=\mathrm{PH} / \mathrm{P}=\mathrm{SH} / \mathrm{S}=0.85$. Under these assumptions, simulated

\footnotetext{
${ }^{1}$ The sales-acquisitions ratios are based on adjusted HMDA sales, described in appendix A.

${ }^{2}$ In our Mortgage System, M\&A at mortgage companies is estimated as a residual (negative one multiplied by M\&A at all other types of mortgagees). During the 1998-2004 period, estimated M\&A at mortgage companies was relatively small; as a result, the results are not altered materially by ignoring M\&A.
} 
RMDO (shown in lines 13 and 14) increased an average of about $\$ 30$ billion per year from 1998 to 2004, or a total of more than $\$ 200$ billion.

The assumptions that underlie the second simulation are perhaps more realistic: we assume that mortgage companies that do not report to HMDA sell all of the loans they originate and do not purchase any loans. That is, $\mathrm{PH}=\mathrm{P}$ and $\mathrm{S} \equiv \mathrm{SH}+\mathrm{SN}=\mathrm{SH}+\mathrm{ON}$ (by assumption, $\mathrm{SN}=\mathrm{ON}$, where $\mathrm{SN}$ and $\mathrm{ON}$ are sales and originations at non-HMDA reporters). Under these assumptions, net acquisitions at all mortgage companies equals $\mathrm{OH}+\mathrm{PH}-\mathrm{SH}$, nullifying the need for a blow-up factor. The simulation results, shown in lines 16 to 20, imply an increase in RMDO of about $\$ 25$ billion per year from 1998 to 2004; at the end of 2004, simulated RMDO is $\$ 172$ billion more than FOF's published level. The actual and simulated paths for RMDO are shown in figure D-1.

As a caveat, the results depend crucially on a number of assumptions: that the adjustments to HMDA sales are accurate, that the repayment rate at mortgage companies is the same as at banks and thrifts, and that the published value of RMDO at mortgage companies in 1997:Q4 is correct. Consequently, the simulations are only suggestive, and do not provide an adequate basis for revising the FOF series.

In the future, FOF hopes to develop data sources to help estimate RMDO at mortgage companies. Potential data sources include regulatory filings that some mortgage companies make to the SEC and FFIEC, and the annual reports of some of the largest mortgage companies. ${ }^{3}$

\footnotetext{
${ }^{3}$ Specifically, some of the largest mortgage companies are part of publicly traded corporations, and their assets may be measurable from data in SEC filings. In addition, some mortgage company subsidiaries of bank holding companies file a regulatory report with limited balance sheet information (FRY11), which may provide information on their holdings. FOF is looking into these potential data sources. Other parts of the FOF accounts already capture some of the assets of mortgage companies. For instance, the regulatory reports filed by banks and thrift institutions include the mortgage assets of mortgage companies that are direct subsidiaries of the reporting institution (i.e. the bank or thrift owns more than 50 percent of the mortgage company). Some finance companies originate 1-4 mortgages and the finance company sector of the FOF accounts probably includes at least some of those assets through the FRB's Finance Company Survey. Nevertheless, we know of no regulatory or survey data source for mortgage companies that are indirect subsidiaries of banks or thrift institutions (i.e. the bank or thrift owns less than 50 percent of the mortgage company) or for independent mortgage companies that are not publicly traded or part of a finance company. Apart from their annual reports, we do not have a way of measuring the assets of these mortgage companies; however, these companies are likely to be relatively small.
} 


\section{Figure D-1}

Published and Simulated Levels of Regular MDO at Mortgage Companies

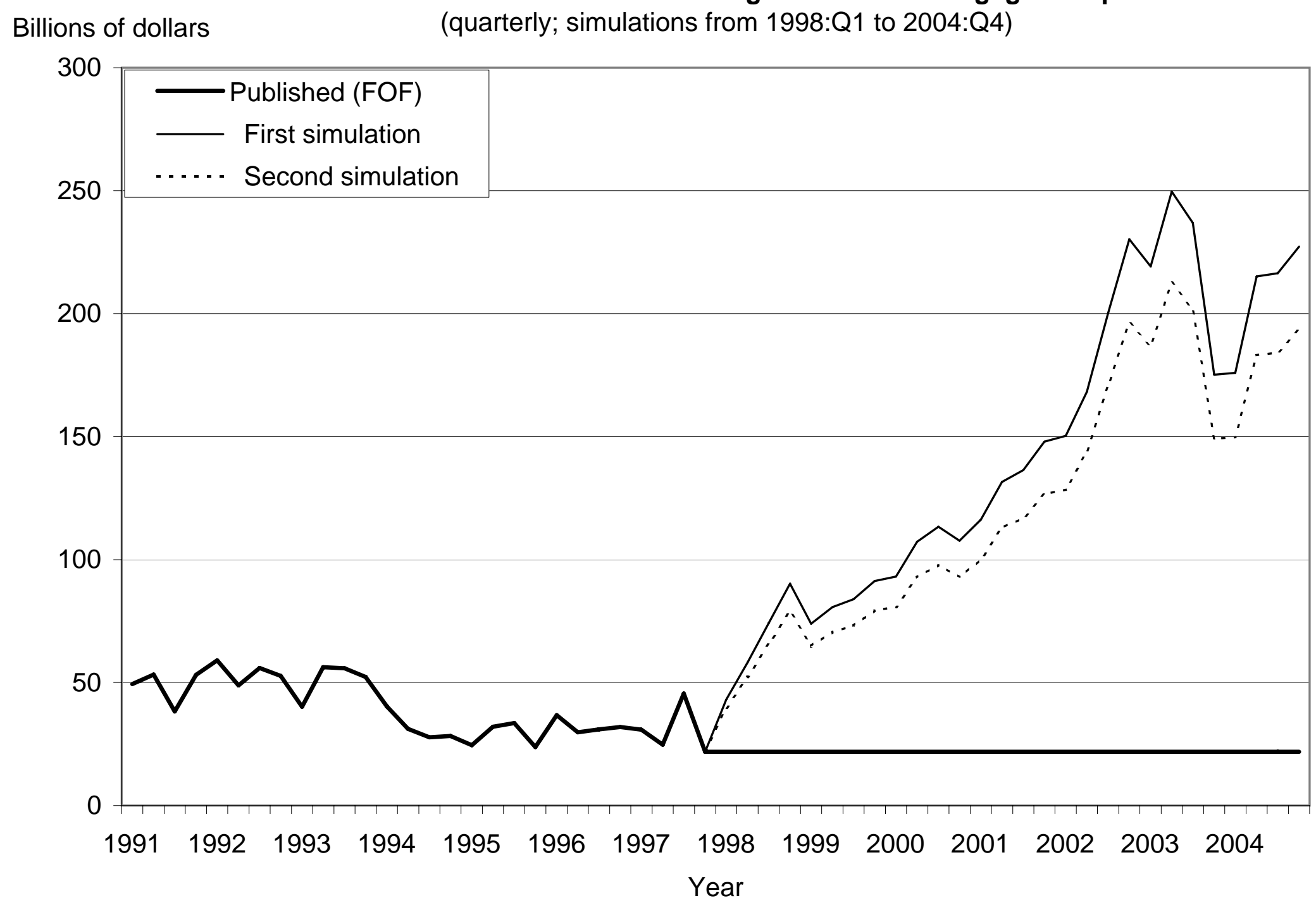


Table D-1

Repayments and Regular Mortgage Debt Outstanding at Mortgage Companies

(billions of dollars, except where noted)

\begin{tabular}{|c|c|c|c|c|c|c|c|c|c|c|}
\hline & & 1991 & 1992 & 1993 & 1994 & 1995 & 1996 & 1997 & 1998 & 1999 \\
\hline & \multicolumn{10}{|l|}{ The Implied Repayment Rate at Mortgage Companies } \\
\hline (1) & Originations (HMDA) & 134.6 & 236.1 & 466.3 & 242.7 & 187.4 & 234.5 & 275.9 & 505.1 & 394.9 \\
\hline (2) & Purchases (HMDA) & 40.0 & 83.6 & 78.6 & 53.0 & 54.6 & 73.3 & 86.7 & 215.4 & 112.9 \\
\hline (3) & Adjusted sales (HMDA with our adjustments) & 150.2 & 282.5 & 467.4 & 282.1 & 187.8 & 255.2 & 311.5 & 649.1 & 487.3 \\
\hline (4) & Net Acquisitions [(1)+(2)-(3)] & 24.4 & 37.3 & 77.5 & 13.6 & 54.2 & 52.7 & 51.2 & 71.4 & 20.6 \\
\hline (5) & Assuming HMDA coverage of mortgage companies is 85 percent & & & 91.2 & 16.0 & 63.8 & 62.0 & 60.2 & 84.0 & 24.2 \\
\hline (7) & Regular mortgage debt outstanding, end of year (FOF) & 53.1 & 52.7 & 52.2 & 28.3 & 23.8 & 32.0 & 21.8 & 21.8 & 21.8 \\
\hline (8) & Change $[(7)-(7)\{t-1\}]$ & 11.3 & -0.4 & -0.5 & -24.0 & -4.5 & 8.2 & -10.2 & 0.0 & 0.0 \\
\hline (9) & Implied repayments [(5)-(8)] & & & 91.6 & 39.9 & 68.3 & 53.8 & 70.3 & 84.0 & 24.2 \\
\hline (10) & Implied repayment rate $[(9) /(7)\{t-1\}]$ & & & 1.7384 & 0.7647 & 2.4158 & 2.2625 & 2.1997 & 3.8512 & 1.1090 \\
\hline & \multicolumn{10}{|l|}{$\begin{array}{l}\text { First Simulation: the repayment rate at mortgage companies is } \\
\text { set equal to the repayment rate at depository institutions and } \\
\text { HMDA coverage of mortgage companies is } 85 \text { percent }\end{array}$} \\
\hline (11) & Assumed repayment rate & & & & & & & & 0.3154 & 0.2804 \\
\hline (12) & Implied repayments at mort. comp. $\left[(11)^{\star}(13)\{t-1\}\right.$, sum of quarterly $\left.)\right]$ & & & & & & & & 15.7 & 23.1 \\
\hline (13) & Implied MDO, end of year $[(13)\{t-1\}+(5)-(12)]$ & & & & & & & 21.8 & 90.2 & 91.2 \\
\hline (14) & Change $[(13)\{t\}-(13)\{t-1\}]$ & & & & & & & & 68.3 & 1.1 \\
\hline (15) & MDO: simulated - published level [(13)-(7)] & & & & & & & & 68.3 & 69.4 \\
\hline & \multicolumn{10}{|l|}{$\begin{array}{l}\text { Second Simulation: same repayment rate as first simulation; } \\
\text { assume non-HMDA reporters sell all of the loans they originate }\end{array}$} \\
\hline (16) & Net Acquisitions [(1)+(2)-(3)] & & & & & & & & 71.4 & 20.6 \\
\hline (17) & Implied repayments at mort. comp. $\left[(11)^{\star}(18)\{\mathrm{t}-1\}\right]$ & & & & & & & & 14.2 & 20.3 \\
\hline (18) & Implied MDO, end of year [(18)\{t-1\}+(16)-(17)] & & & & & & & 21.8 & 79.0 & 79.3 \\
\hline (19) & Change $[(18)\{t\}-(18)\{t-1\}]$ & & & & & & & & 57.2 & 0.3 \\
\hline (20) & MDO: simulated - published level [(18)-(7)] & & & & & & & & 57.2 & $\mathbf{5 7 . 5}$ \\
\hline
\end{tabular}


Table D-1

Repayments and Regular Mortgage Debt Outstanding at Mortgage Companies

(billions of dollars, except where noted)

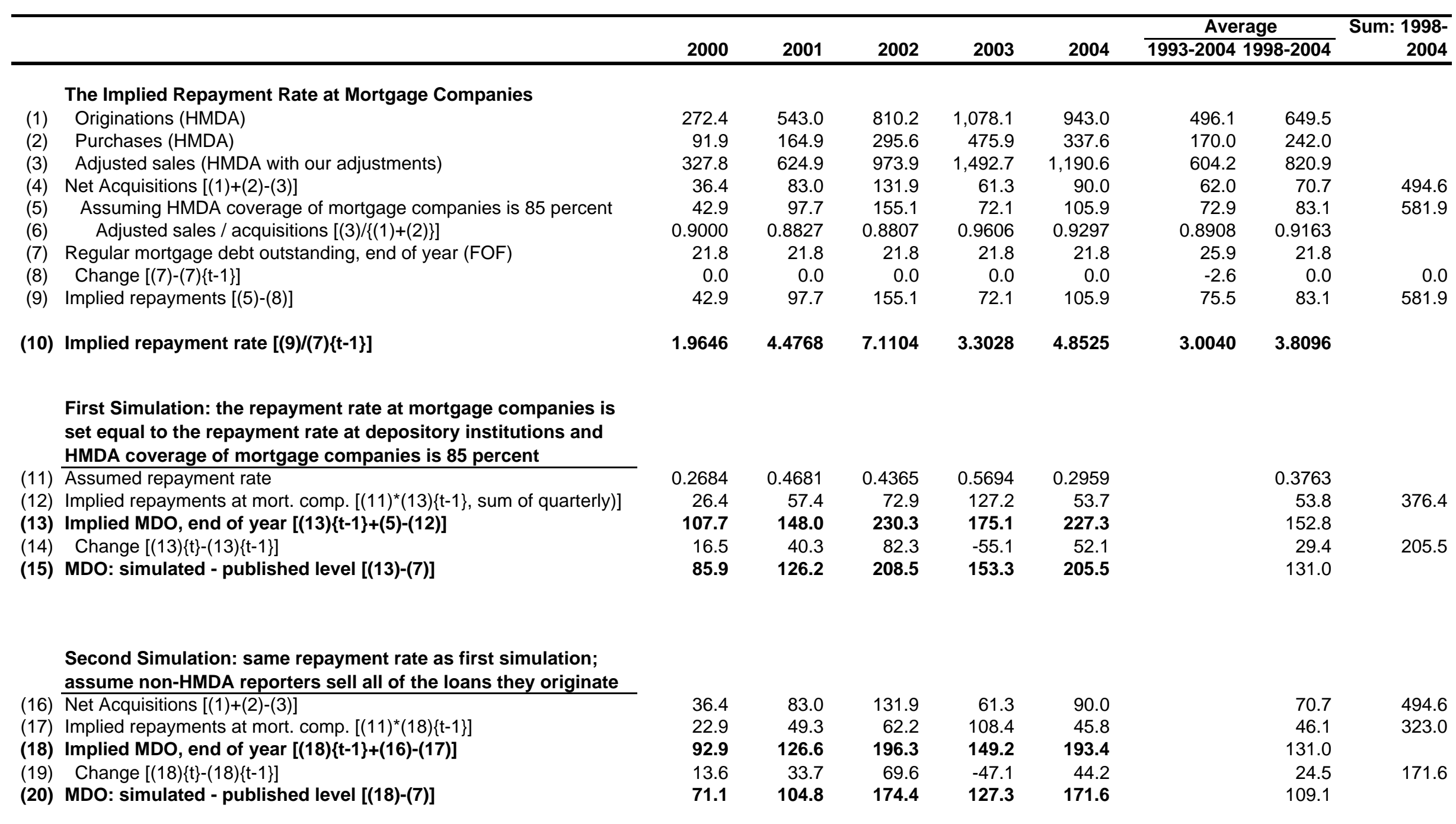




\section{Appendix E \\ The Decomposition of Repayments}

We define two general types of repayments in our mortgage system, those resulting from refinancings and all other types of repayments.

\section{Repayments Resulting from Refinancings}

By definition, refinance repayments $\left(\mathrm{R}^{r}\right)$ equal refinance originations $\left(\mathrm{O}^{r}\right)$ minus the gross cash-out (GC). The gross cash out share, $\sigma$, is defined as $\left(\mathrm{GC} / \mathrm{O}^{r}\right)$. Thus, repayments resulting from refinancings equal

$$
\mathrm{R}^{\mathrm{r}}=\mathrm{O}^{\mathrm{r} *}(1-\sigma)
$$

Conventional loans have a much larger gross cash-out share than government-backed loans; thus, we estimate (1) separately for those two types of loans. The gross cash-out share for conventional loans, denoted $\sigma^{\mathrm{c}}$, is based on data published by Freddie Mac, which include only conforming loans. ${ }^{1}$ We assume that the gross cash out share for the loans purchased by Freddie Mac is representative of all types of conventional loans, including subprime regular mortgages and jumbos. During the 1991-2004 period, $\sigma^{c}$ averaged close to 0.15 (line 123 of table 1). For FHA and VA refinancings, we assume a fixed gross cash-out share of 0.03 , a rough estimate of the amount required to cover closing costs. ${ }^{2}$

Thus, total repayments resulting from refinancings (line 84 of table 1) equal

$$
\mathrm{R}^{\mathrm{r}}=\left(\mathrm{O}^{\mathrm{r}}-\mathrm{Og}^{\mathrm{r}}\right) *\left(1-\sigma^{\mathrm{c}}\right)+\mathrm{Og}^{\mathrm{r}} *(1-0.03)
$$

where $\mathrm{Og}^{\mathrm{r}}$ denotes FHA and VA-guaranteed refinance originations.

We calculate non-refinance repayments $\left(\mathrm{R}^{\mathrm{n}}\right)$ as a residual:

$$
\mathrm{R}^{\mathrm{n}}=\mathrm{R}-\mathrm{R}^{\mathrm{r}}
$$

\footnotetext{
${ }^{1} \varphi$ is calculated from loans in which Freddie purchased both the loan paid off at the time of the refinance and the new loan. The shares were calculated from data published by Freddie Mac in its quarterly report on cash outs from refinancing, available at http://www.freddiemac.com/news/finance/data.html. Our gross cash out share is equal to Freddie's estimate of cash outs from refinancing and home equity debt consolidation (labeled as "Volume of cash-out and $2^{\text {nd }}$ mortgages/HELOC consolidation" in Freddie's tables) divided by Freddie's estimate of conventional, prime, refinance originations. The data needed to calculate the gross cash out share are not available before 1993; we estimate the gross cash out shares before 1993 based on an equation that includes lags of changes in the house price index published by the Office of Federal Housing Enterprise Oversight and the difference between the thirty-year fixed mortgage rate and the weighted-average coupon rate (described below). In addition, the Freddie data are only available annually from 1993 to 1998; we interpolate the quarterly gross cash out shares over that period. ${ }^{2}$ Our assumed gross cash out share for FHA and VA loans is based on discussions with people at HUD and the VA.
} 
We estimate three components of non-refinance repayments: amortization, sellers' debt cancellation, amortization, and unscheduled payments. The repayments discrepancy equals total non-refinance repayments minus these three components.

Amortization: is calculated using the following variables: an estimate of the weightedaverage coupon rate (WAC) on outstanding mortgages developed by staff at the Federal Reserve Board, ${ }^{3}$ the average term to maturity based on data from Citigroup, ${ }^{4}$ and the level of RMDO at the beginning of the quarter (see lines 106 to 111 of table 1). During the 1991-2004 period, amortization averaged a bit more than 1-1/2 percent of beginning-ofyear RMDO per year.

We benchmark our WAC in 1992:Q1 to a series from the Bureau of Economic Analysis (BEA), "the effective rate of interest on mortgage debt outstanding.", Quarterly changes in the WAC from 1992:Q2 onward evolve according to the following: mortgages that carried through from the previous quarter to the current quarter, mortgages that refinanced in the current quarter, mortgages for home purchase, and cash-out refinances in the current quarter. To calculate the evolution of the WAC, we use our estimates of home purchase and refinance originations and the gross cash out, the median ratio of the old to the new mortgage rate on refinance loans (from Freddie Mac's quarterly cash out report), and the thirty-year fixed mortgage rate (from Freddie's weekly survey of mortgage lenders). Figure E-1 shows our estimate of the WAC along with BEA's "effective rate of interest," which is the average coupon rate that BEA applies to all 1-4 mortgage debt in order to estimate mortgage interest payments.

Sellers' debt cancellation measures the repayment of the sellers' mortgage outstanding at the time of sale. We estimate both total sellers' debt cancellation and a subcomponent of the total, debt cancellation resulting from foreclosure (the latter is described in the next section).

We use a two-step procedure to estimate total sellers' debt cancellation. First, for homes sold in the current quarter, we estimate the originations of regular mortgages used to finance the purchase of those homes in previous quarters. Second, we track the evolution of that mortgage debt from the time of purchase to the time of sale.

The calculation of the originations used to finance home purchases requires the following variables:

(1) The distribution of when homes sold in the current quarter were acquired. We estimated a fixed vector, denoted $\gamma$, based on American Housing Surveys (AHS) from 1985 to 2003. The ith element of $\gamma$ is the share of homes sold in the current

\footnotetext{
${ }^{3}$ Andreas Lehnert at the Federal Reserve developed the method to estimate the WAC.

${ }^{4}$ From the Citigroup Mortgage Index (Copyright 2005, Citigroup Index LLC, all rights reserved.) We calculate a weighted average of the average term to maturity of mortgages securitized by Ginnie Mae, Fannie Mae, and Freddie Mac (30-year, 15-year, and ARM loans). The weights are based on the dollar value of each category's share in the total.

${ }^{5}$ The BEA series is available on its website.
} 
quarter that were purchased $\mathrm{i}$ quarters earlier, $\mathrm{i}=1, \ldots, 160$. The AHS data used to estimate the vector pertain only to owner-occupied homes; we assume that the same distribution applies to rental and vacant homes.

(2) The number of existing homes sold at time $t$, denoted $\mathrm{N}_{\mathrm{t}}$, from the National Association of Realtors (NAR).

(3) The price of homes purchased at $t-i$, a weighted average of the prices for new homes from Census and existing homes from the NAR, denoted $\mathrm{P}_{\mathrm{t}-\mathrm{i}}$.

(4) The mortgaged share of homes purchased at $t-i$, which we estimate based on data from the 2000 Residential Finance Survey (RFS) and AHS surveys from 1985 to 2003, denoted $\mathrm{m}_{\mathrm{t}-\mathrm{i}}$.

(5) The loan-to-price ratio of mortgaged purchases, estimated as a weighted-average of the LTPs for conventional loans (FHFB), FHA loans, and VA-guaranteed loans, denoted $\delta_{\mathrm{t}-\mathrm{i}}$.

(6) The ratio of the average price of mortgaged homes to the average price of all homes, based on the 2000 RFS, denoted $\varphi$, which we assume is fixed over the sample period at 1.04 .

(7) The share of the number of homes purchased with an assumed mortgage based on AHS surveys from 1985 to 2003, denoted $\varepsilon_{\mathrm{t}-\mathrm{i}}$. The assumed share trended down from a bit more than 3 percent in 1991 to 0.5 percent in recent years.

(8) The ratio of the value of homes purchased implied by our home purchase originations, the mortgaged share, and the LTP ratio and value based on data from Census and the NAR, denoted $\Pi_{t-i}$ (discussed in section 5 of the main paper; see lines 15 to 17 of table 1). П averaged 0.90 from 1991 to 2004 .

Let $\mathrm{O}^{\mathrm{p}}\{\mathrm{t}, \mathrm{t}-\mathrm{i}\}$ denote originations at $\mathrm{t}-\mathrm{i}$ to finance the purchase of homes that were sold at t. Based on the variables definitions above,

$$
\mathrm{O}^{\mathrm{p}}\{\mathrm{t}, \mathrm{t}-\mathrm{i}\}=\left(\gamma_{\mathrm{i}} * \mathrm{~N}_{\mathrm{t}}\right) *\left(\mathrm{P}_{\mathrm{t}-\mathrm{i}} * \varphi\right) *\left(\mathrm{~m}_{\mathrm{t}-\mathrm{i}} * \delta_{\mathrm{t}-\mathrm{i}}\right) *\left(1-\varepsilon_{\mathrm{t}-\mathrm{i}}\right) * \Pi_{\mathrm{t}-\mathrm{i}}
$$

We track each cohort of mortgage debt, $\mathrm{O}^{\mathrm{p}}\{\mathrm{t}, \mathrm{t}-\mathrm{i}\}$, for $\mathrm{t}$ beginning in 1990:Q1 and $\mathrm{i}=1, \ldots 160$. Define $\mathrm{D}\{\mathrm{t}, \mathrm{t}-\mathrm{i}\}_{\mathrm{t}-\mathrm{j}}$ as the unpaid balance of mortgages at $\mathrm{t}-\mathrm{j}$ for homes sold at $\mathrm{t}$ that were purchased at $\mathrm{t}-\mathrm{i}, 0 \leq \mathrm{j} \leq \mathrm{i}$. Thus, $\mathrm{D}\{\mathrm{t}, \mathrm{t}-\mathrm{i}\}_{\mathrm{t}-\mathrm{i}} \equiv \mathrm{O}^{\mathrm{p}}\{\mathrm{t}, \mathrm{t}-\mathrm{i}\}$. The evolution of $\mathrm{D}$ from the time of purchase to the time of sale depends on amortization, unscheduled payments, and cash outs associated with refinancing. We estimate amortization based on our WAC and the average term of outstanding mortgages (described above). We have not been able to find any hard data on unscheduled payments; as mentioned in the main paper, we estimate them for now as 0.1 percent at an annual rate of beginning-of-quarter RMDO. The increase in mortgage debt associated with cash outs from refinancing at $t-i+1$ is equal to the gross cash out share in that period multiplied by $\mathrm{D}\{\mathrm{t}, \mathrm{t}-\mathrm{i}\}_{\mathrm{t}-\mathrm{i}}$.

Our estimate of sellers' debt cancellation begins in 1990:Q1. Because $\gamma$ has 160 components, we require time series beginning in 1950 for most of the variables used in the calculation; however, all none of the series goes back that far. We used a number of proxies to extend the series back; a detailed description is available on request. 
Foreclosure Charge-offs: The estimates are based, in part, on quarterly data from the MBA on the percent of outstanding mortgages in foreclosure and the percent for which foreclosure was started. ${ }^{6}$ Through the end of 1999, the MBA also published the total number of regular mortgage loans outstanding. We calculated from these data the total number of loans in foreclosure and the number that entered foreclosure proceedings. Exits from foreclosure equal the change in the number of loans in foreclosure minus the number that entered foreclosure. Based on conversations with people at FHA, VA, and at a few large servicers, we assume a cure rate of 70 percent. Thus, we assume that 30 percent of exits are foreclosures (see lines 112 to 122 of table 1).

We also developed estimates of the average acquisition price and debt cancellation of loans foreclosed in the current period. Charge-offs resulting from foreclosure equal average debt cancellation per foreclosure sale multiplied by the number of loans that were foreclosed. Because MBA's series on the total number of mortgage loans outstanding stops at the end of 1999, we can only estimate foreclosures using this method through the end of that year. MBA's series on the total number of loans outstanding is not available after 1999 .

\footnotetext{
${ }^{6}$ From MBA’s "National Delinquency Survey."
} 
Figure E-1

Measures of the Weighted Average Coupon Rate

Percent

(1991:Q1-2004:Q4)

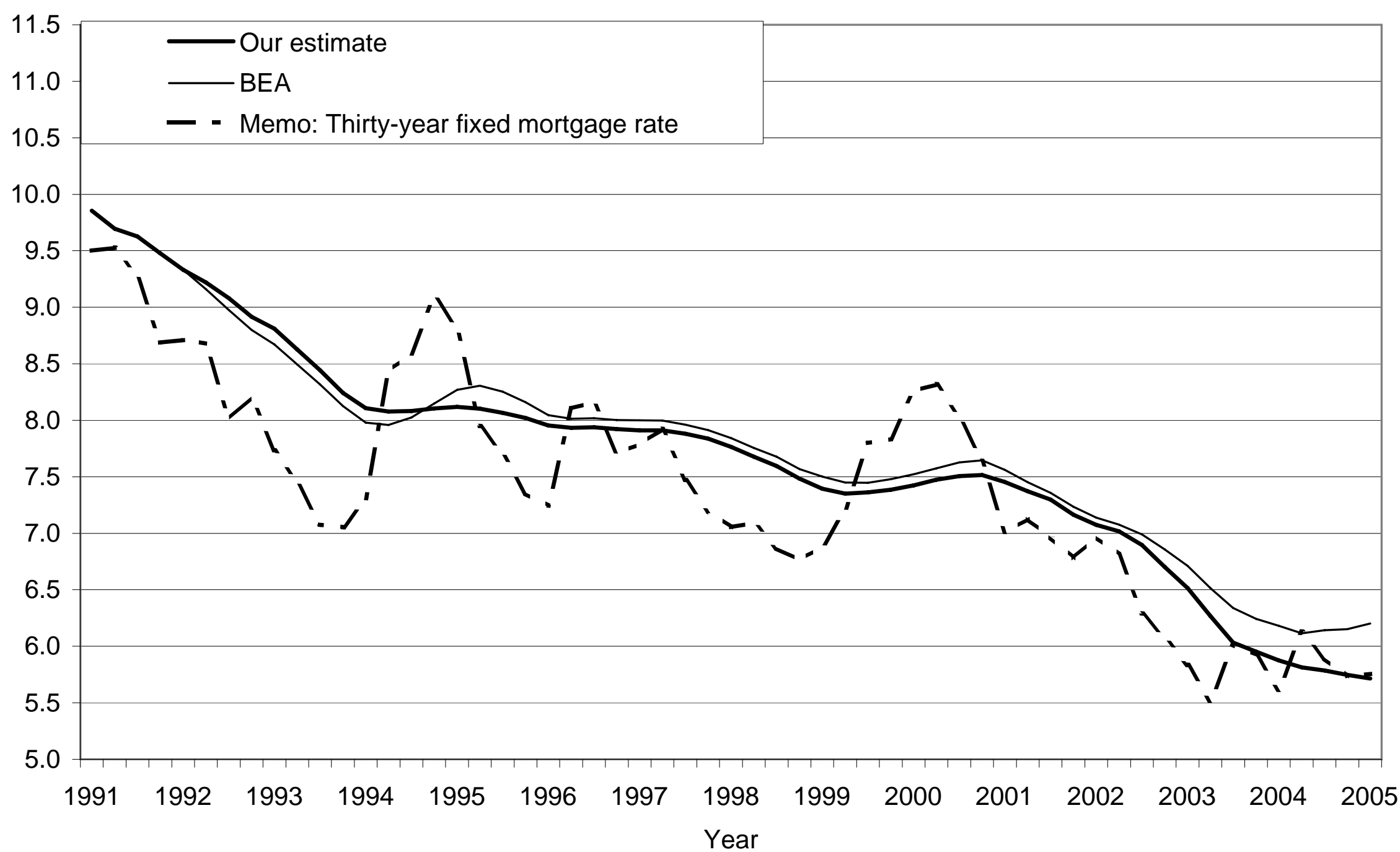

See appendix $E$ for a description of the series. The thirty-year fixed mortgage rate is from Freddie Mac 


\section{Appendix F Mortgage Originations to Finance Home Purchases and to Refinance Existing Loans}

HMDA respondents report whether a mortgage was used to finance a home purchase or to refinance an existing loan. To decompose our total originations into purchase and refinance originations, we first calculate the HMDA refinance share of originations at each of the five types of originators and assume that refinance shares are the same at HMDA and non-HMDA reporters. For example, line 139 of table 1 shows the refinance share of HMDA originations at commercial banks. The HMDA refinance share multiplied by our estimate of originations at commercial banks (line 127) equals total refinance originations at banks (line 140). Total refinance originations (line 10) equals the sum at each of the five types of originators.

The refinance share in the HMDA data is considerably larger than the refinance shares reported by Freddie Mac and the MBA. The HDMA data allow us to calculate refinance shares for both mortgage applications and originations; the refinance shares from Freddie Mac and the MBA are available only for mortgage applications. For example, for 1991 to 2004, the HMDA refinance share of originations averages about 50-1/2 percent. In contrast, the refinance share of mortgage applications from the MBA averages 40-1/2 percent, and from Freddie Mac it averages 42-1/2 percent (see figure F-1 and lines 44 to 49 of table 1$)$.

Freddie surveys 125 lenders every month, asking each to report the share of the number of refinance applications to the total number of applications received. Freddie's published refinance share is an unweighted mean of the survey responses. However, large lenders tend to have a larger refi share than small lenders, which, in itself, would impart a downward bias to the overall refi share. ${ }^{1}$

The MBA surveys about twenty large lenders (depository institutions and mortgage companies) each week; like Freddie, it gathers data on mortgage applications, not originations. The MBA survey allows us to calculate the refinance share of either the number or the dollar volume of applications. For reasons we have not been able to identify, the HMDA refinance applications shares are considerably larger than the MBA refinance shares for both the number and the dollar volume of applications.

We also compared the refinance share of FHA originations (based on data from the FHA, which cover all FHA loans) to the HMDA data's refinance share for FHA loans (which in recent years has covered about 95 percent of FHA loans). The two shares, shown in lines line 50 and 51 of table 1, are almost identical. Thus, at least in the case of FHA loansin which the actual refinance share of originations is known - there is no evidence that

\footnotetext{
${ }^{1}$ This result is from an unpublished paper by Frank Nothaft and Yan Chang ("De-Mystifying the RefiShare Mystery," Freddie Mac, 2005) in which the authors make a strong case that HMDA offers a better measure of the refi share of originations (measured in dollars) than either the Freddie Mac or the MBA series.
} 
the HMDA data overstate the refinance share. ${ }^{2}$ Nevertheless, FHA loans are not representative of the universe: They constitute less than 10 percent of total originations, and HMDA covers a much larger portion of FHA loans than of conventional loans.

One possible explanation of the gap between the HMDA refinance share and the shares from Freddie Mac and the MBA is that the HMDA share contains an upward bias because it does not cover small lenders, who tend to have a lower refinance share than large lenders. However, the data do not support that argument: Freddie's refinance share covers both large and small lenders, whereas MBA's covers only large lenders, yet those two refinance shares are almost identical over time. Another possibility is that refinance shares based on applications contain a downward bias because refinance applications are more likely than purchase applications to result in a closing. However, the HMDA data show just the opposite: The likelihood of a purchase application closing was about 73 percent during the 1991-2001 period, 13 percentage points more than the closing rate on refinance applications.

Because the HMDA data cover a larger share of the universe than either the Freddie Mac or MBA series, are based on well-defined reporting standards, and offer data on the refinance share of originations as opposed to applications, we believe that they provide the most accurate estimate of the refinance share of originations.

\footnotetext{
${ }^{2}$ The refinance shares for VA-guaranteed loans based on data from the VA and HMDA also are almost identical (VA loans accounted for 2-1/2 percent of HMDA originations in the 1993-2004 period).
} 
Figure F-1

Measures of Mortgage Refinancings as a Share of Originations

Ratio

(1991:Q1-2004:Q4)

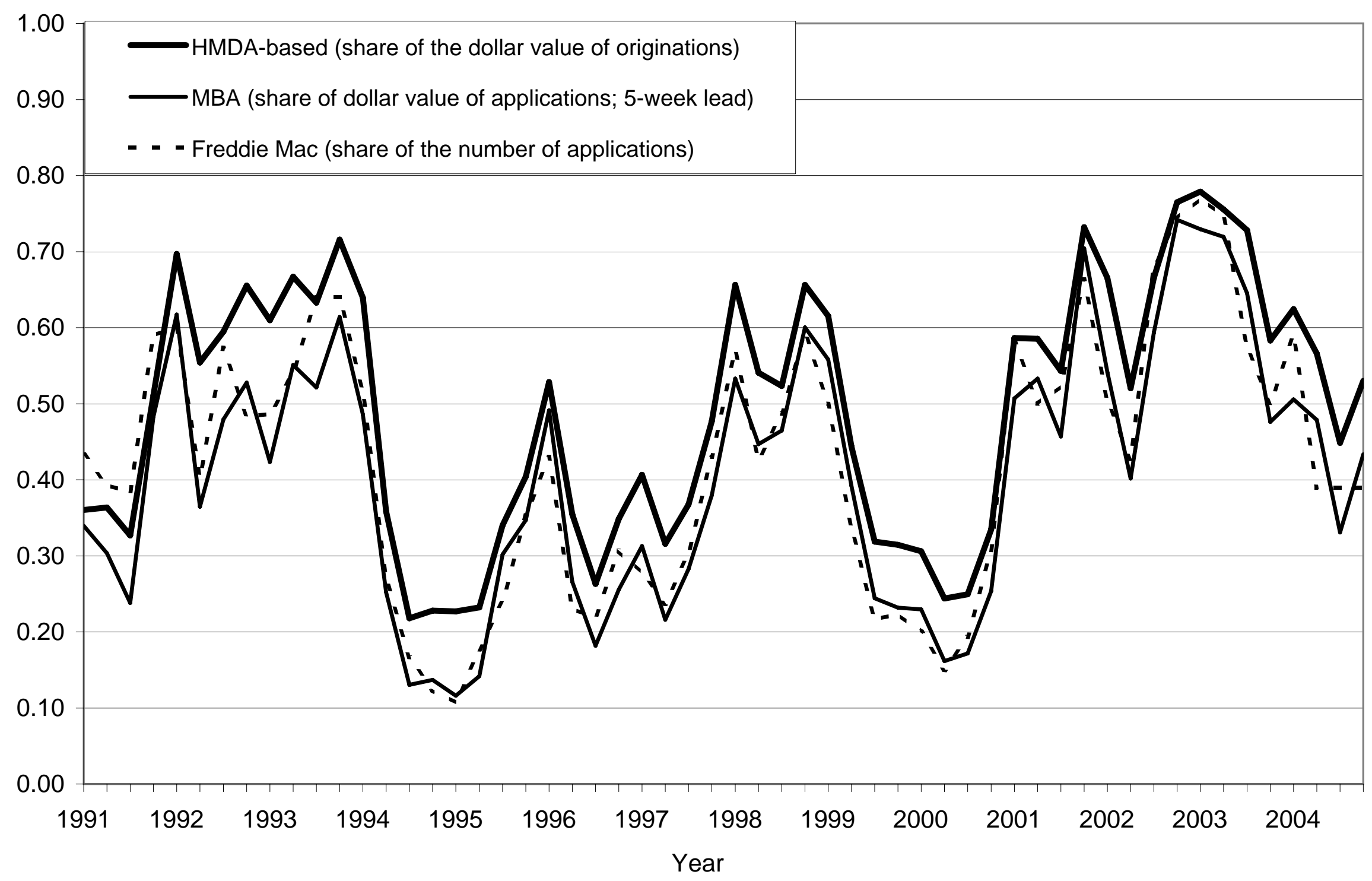




\section{Bibliography}

Bendt, Douglas L, et al, "Prepayment Analysis of Nonagency Mortgage Backed Securities," in The Handbook of Nonagency Mortgage Backed Securities: Second Edition, edited by Fabozzi, et al, Wiley, 2001, pp. 195-206.

Bruskin, Eric, and Sykes, David, "The Nonagency Mortgage Market: Background and Overview," in The Handbook of Nonagency Mortgage Backed Securities: Second Edition, edited by Fabozzi, et al, Wiley, 2001, pp. 3-33.

Bunce, Harold L., and Scheessele, Randall M., “The GSEs’ Funding of Affordable Loans,” Housing Finance Working Paper Series, U.S. Department of Housing and Urban Development, No. HF-001, 1996.

Bunce, Harold L., and Scheessele, Randall M., “The GSEs’ Funding of Affordable Loans: A 1996 Update,” Housing Finance Working Paper Series, U.S. Department of Housing and Urban Development, No. HF-005, 1998.

Chicago Title and Trust Company, "Who’s Buying Homes in America,” various issues.

Fan, Yizhong, and Rogers, Pete, "Data Processing and Prepayment Modeling of Whole Loans," in The Handbook of Nonagency Mortgage Backed Securities: Second Edition, edited by Fabozzi, et al, Wiley, 2001, pp. 221-249.

Fiens, Judith D; Herbert, Christopher E.; and Connolly, Christine, "Exploratory Study of the Accuracy of Home Mortgage Disclosure Act (HMDA) Data, Final Report,” Abt Associates Inc., April 1999.

Guide to the Flow of Funds Accounts, Volumes 1 and 2, Board of Governors of the Federal Reserve System, 2000.

Manchester, Paul B.; Neal, Sue George; and Bunce, Harold L., "Characteristics of Mortgages Purchased by Fannie Mae and Freddie Mac, 1993-95,” Housing Finance Working Paper Series, U.S. Department of Housing and Urban Development, No. HF003, 1998.

Nothaft, Frank E., and Chang, Yan, “De-Mystifying the Refi-Share Mystery,” unpublished paper, Freddie Mac, 2005.

Patruno, Gregg N., “Mortgage Prepayment Modeling: II,” in The Handbook of Mortgage Backed Securities, edited by Fabozzi, Frank J., Probus, 1995, pp. 143-168.

Scheessele, Randall M., "HMDA Coverage of the Mortgage market," Housing Finance Working Paper Series, U.S. Department of Housing and Urban Development, No. HF007, 1998. 
Schorin, Charles, and Gordon, Mark, “Mortgage Prepayment Modeling: I,” in The Handbook of Mortgage Backed Securities, edited by Fabozzi, Frank J., Probus, 1995, pp. 127-141.

Westhoff, Dale, et al, "Prepayments of Non-Standard Whole-Loan Mortgage Securities," in The Handbook of Nonagency Mortgage Backed Securities: Second Edition, edited by Fabozzi, et al, Wiley, 2001, pp. 207-220. 